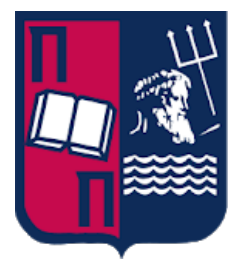

University of Piraeus

School of Information and Communication Technologies

Department of Digital Systems

\title{
Security and Privacy Enhancing Mechanisms for The Android Operating System
}

Doctoral Thesis

Christos Lyvas

Piraues, December 2021 


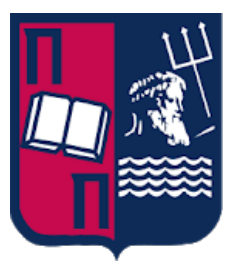

University of Piraeus

School of Information and Communication Technologies

Department of Digital Systems

\section{Security and Privacy Enhancing Mechanisms for The Android Operating System}

Supervisor: Prof. Costas Lambrinoudakis

Advisors: Prof. Christos Xenakis

Asst. Prof. Panagiotis Rizomiliotis

This thesis is submitted for the degree of

Doctor of Philosophy

Piraues, December 2021 


\section{APPROVAL SHEET \\ UNIVERSITY OF PIRAEUS \\ SCHOOL OF INFORMATION AND COMMUNICATION TECHNOLOGIES DEPARTMENT OF DIGITAL SYSTEMS}

This is to certify that the Thesis presented by Christos Lyvas, entitled "Security and Privacy Enhancing Mechanisms for The Android Operating System", submitted in fulfillment of the requirement for the degree of Doctor of Philosophy, complies with the regulation of the University of Piraeus and meets the accepted standards with respect to originality.

Costas Lambrinoudakis

Professor

University of Piraeus

(Supervisor)

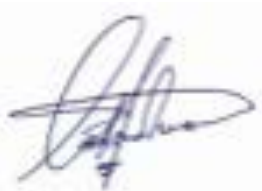

(Signature)

17 December 2021

(Date)

Christos Xenakis

Professor

University of Piraeus

(Advisor)

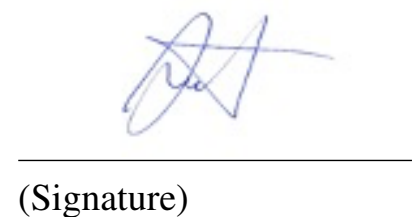

17 December 2021

(Date)

Panagiotis Rizomiliotis

Assistant Professor

Harokopio University of Athens

(Advisor)

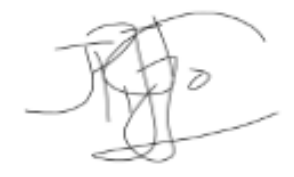

(Signature)

17 December 2021

(Date)

Stefanos Gritzalis

Professor

University of Piraeus

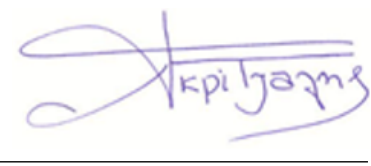

(External Examiner)

(Signature)

17 December 2021

(Date) 
Sokratis Katsikas

Professor

Norwegian University

of Science and Technology

(External Examiner)

Christos Kalloniatis

Associate Professor

University of Aegean

(External Examiner)

Nikolaos Pitropakis

Associate Professor

Edinburgh Napier University

(External Examiner)

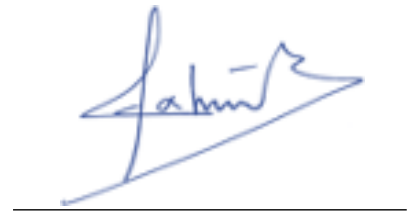

(Signature)

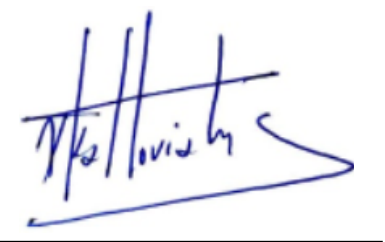

(Signature)

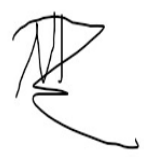

(Signature)
17 December 2021

(Date)

17 December 2021

(Date)

17 December 2021

(Date) 


\section{Declaration}

I hereby declare that except where specific reference is made to the work of others, the contents of this thesis are original and have not been submitted in whole or in part for consideration for any other degree or qualification in this, or any other university. This thesis is my own work and contains nothing which is the outcome of work done in collaboration with others, except as specified in the text and Acknowledgements. I additionally declare that the opinions expressed in this document are my sole responsibility and do not necessarily represent the official position of the University of Piraeus.

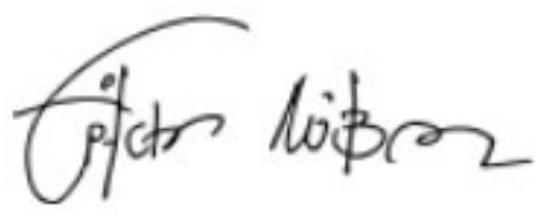

Christos Lyvas

Piraeus, December 7, 2021 

To my grandfather, who used to ask me every day if I got my Ph.D., as well as my parents and Chrys for their tolerance and support. 

"People think of education as something that they can finish."

Isaac Asimov 



\section{Acknowledgements}

There are many people I would like to express my appreciation and gratitude for their contributions to this dissertation and my scientific evolvement over the past years. First, I would like to express my sincere gratitude to my supervisor Professor Costas Lambrinoudakis for his kindness, invaluable mentoring, endless support, guidance, and confidence in me and my potential.

This dissertation would also not have been possible without the support, excellent cooperation, and contribution of Dr. Dimitris Geneiatakis, which was very constructive during my studies.

I would like to thank the advisory committee, Professor Christos Xenakis, and Assistant Professor Panagiotis Rizomiliotis.

I would like to extend my appreciation to Professor Athanasios G. Kanatas and Professor Stefanos Gritzalis for their faith in me and for allowing me to participate in several national and European projects that broadened my horizons to several scientific fields.

Moreover, I would like to thank Associate Professor Christos Kalloniatis and Assistant Professor Konstantinos Maliatsos for their excellent cooperation and guidance during my participation in research projects in recent years.

Finally, I would like to express my appreciation to the Systems Security Laboratory and Telecommunication Systems Laboratory members of the University of Piraeus and particularly Dr. Viktor Nikolaidis for their support and friendship.

Christos Lyvas

Piraeus, December 7, 2021 



\begin{abstract}
The Android platform is the dominant Operating System (OS) for mobile and IoT devices. Its wide distribution is mainly due to the freedom it grants to mobile manufacturers (OEMs) to use it as the primary operating system for their devices. In terms of security, Android's enhanced security model safeguards the end-users from the threats of conventional Operating Systems (macOS, Windows, Linux, etc.). However, Android has vulnerabilities due to its architecture particularities. This thesis focuses on the threats, vulnerabilities and security mechanisms of the Android Operating System (OS).

Initially, the permission-based access control model of the Android Operating System for controlling access to sensitive phone resources and its "relation' to the Android framework's methods for constructing the permission maps has been investigated. The aforementioned "relation" between a framework method and a permission can be found in the Android documentation. However, in addition to the fact that documentation may accidentally lack information, Android features undocumented and hidden API methods. To this direction, this thesis proposes Dypermin, a transparent framework for compiling the Android permission map without requiring any modification to the underlying operating system. To achieve that, Dypermin capitalizes on intrinsic properties of the Android framework, that is, security exceptions during run time and the availability of any protected API method through the Android framework, as well as on the advantages of the Java reflection mechanism. Furthermore, Dypermin, in contrast to other related methods, validates itself as it relies on run time information, meaning that it does not generate false-positive map entries. Dypermin has been evaluated on different Android versions. The results have been compared with those of other proposed methods demonstrating Dypermin's efficacy for compiling the Android permission map for any given version.

Furthermore, it has been demonstrated that the Android's activity and task hijacking attacks may have a significant impact on end users' data confidentiality since malicious applications can deceive end-users and silently gain access to sensitive data. Undoubtedly, such attacks are of vital importance and thus, for their thorough study, a tool named Anactijax has been proposed, used for identifying specific configurations that an application may be vulnerable to. Furthermore, an operating system level defense mechanism named TaskAuth has
\end{abstract}


been proposed, controlling the access to the applications' activities. TaskAuth is transparent to both end-users and developers by leveraging their built-in signatures. The effectiveness of TaskAuth has been evaluated against various vulnerable configurations provided by Anactijax. Results have proved that the proposed solution does not affect Android's task management and that end-users suffer negligible execution overhead.

Finally, the Android intent redirection and intent hijacking attacks were examined. Again this type of attacks can be launched by malicious applications in order to gain access to users' sensitive data. For addressing this type of attacks an operating system-level defense mechanism named IntentAuth has been proposed. IntentAuth allows end-users to explicitly define dynamic policies that specify a trust model for applications allowed to interact with each other. Additionally, upon the user-defined trust model for installed applications, the proposed implementation can verify and encrypt the data transmitted (application to application) during the Android's Inter-Process communication mechanism via implicit Intents. The execution overhead imposed has been proved to be negligible.

Keywords: Android Security, Activity Hijacking Attacks, Task Hijacking Attacks, Task Hijacking Prevention, Activity Hijacking Prevention, Mobile Operating System Security, Mobile Application Security, Mobile Application Privacy, Android Privacy, Intent Redirection Attacks Prevention, Intent Hijacking Prevention, Intent Encryption, Intent Authorization, Intent Authentication, Android Permissions Map, Reflection Technique, Dynamic Analysis 


\section{Пврі́ $\eta \psi \eta$}

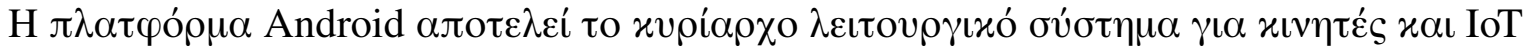

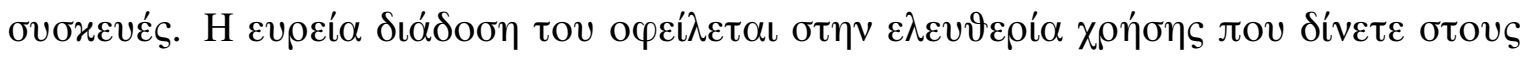

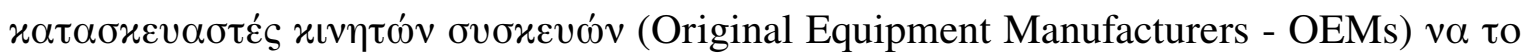

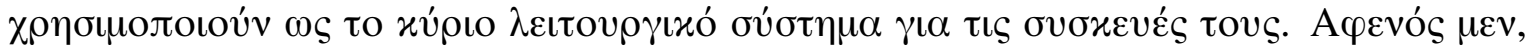

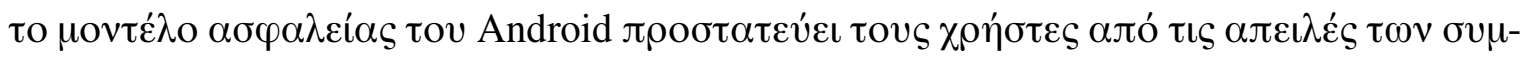

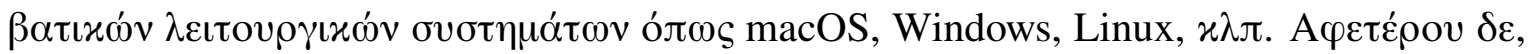

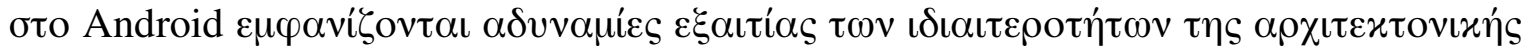

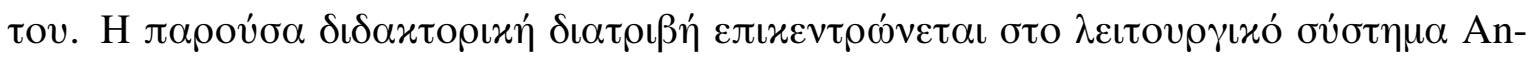

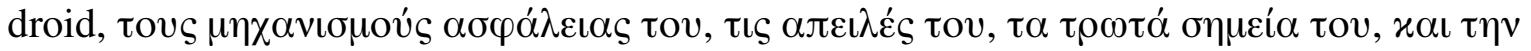

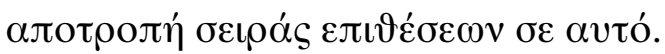

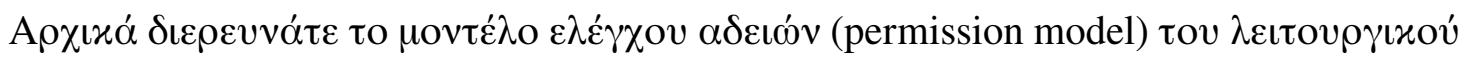

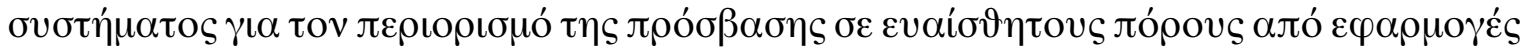

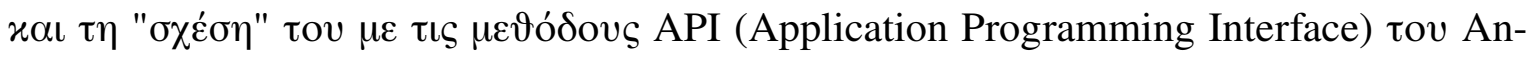

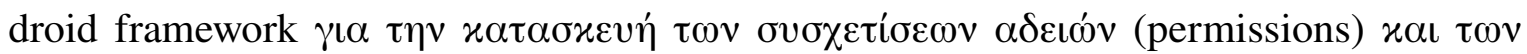

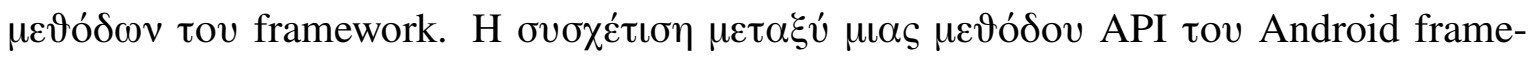

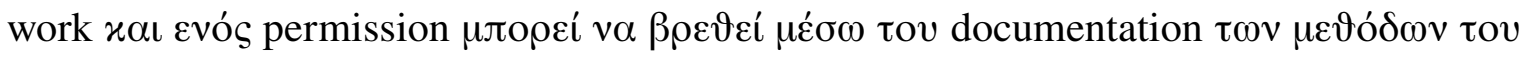

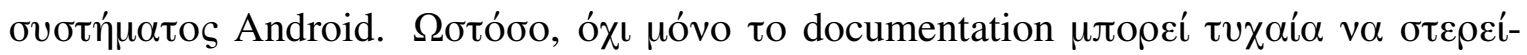

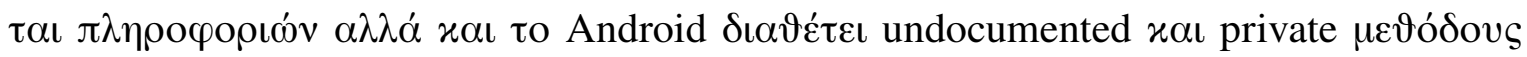

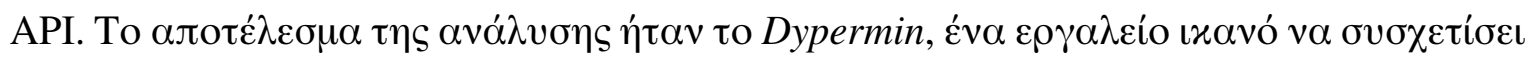

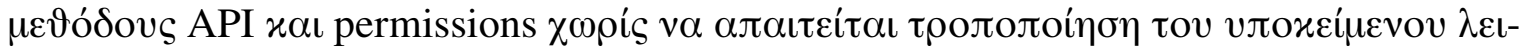

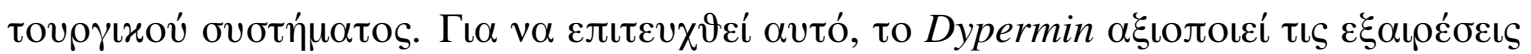

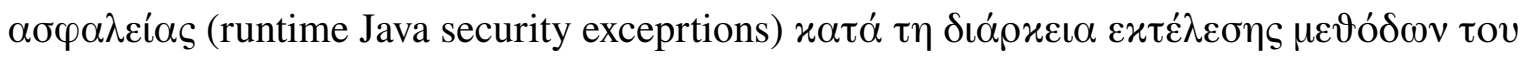

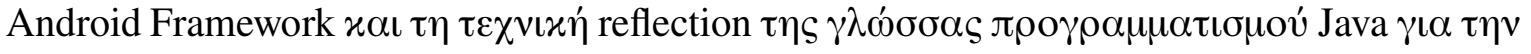

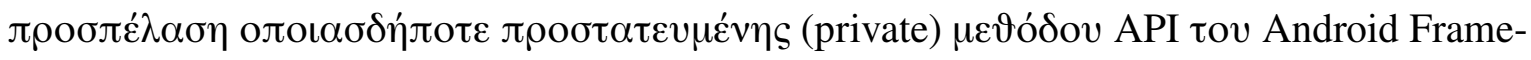

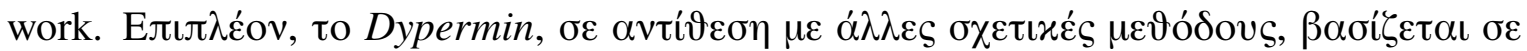

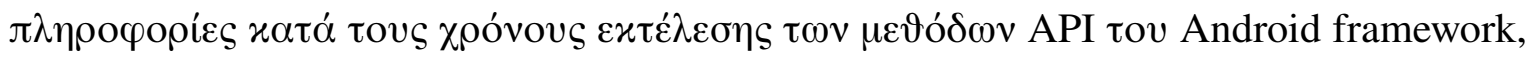

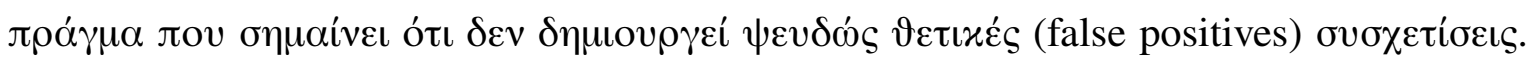

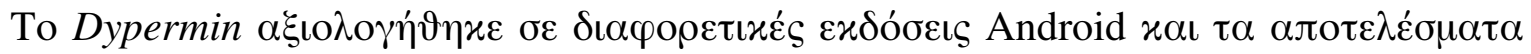




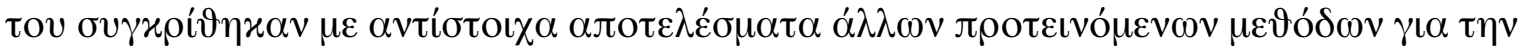

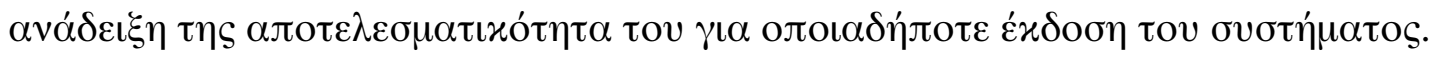

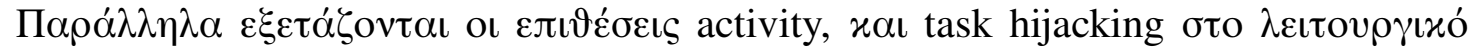

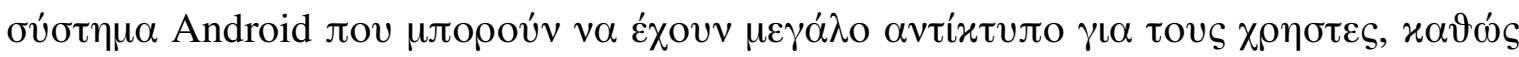

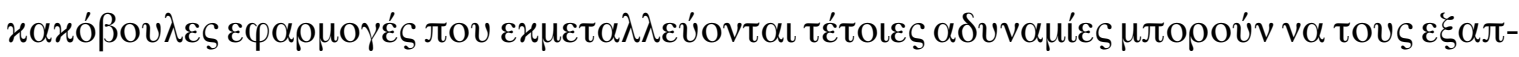

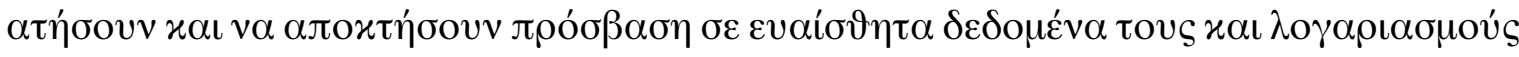

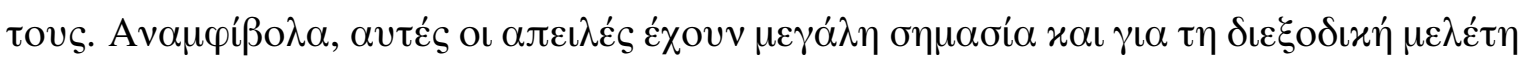

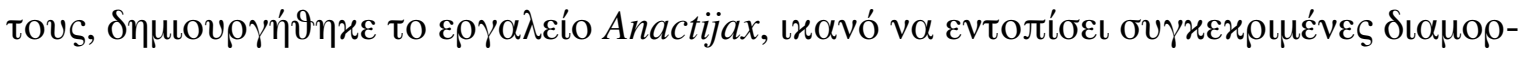

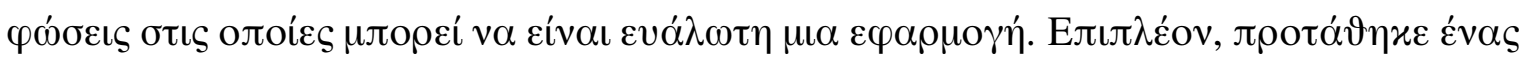

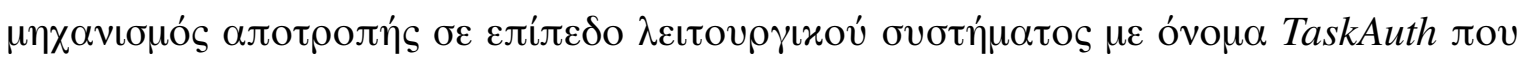

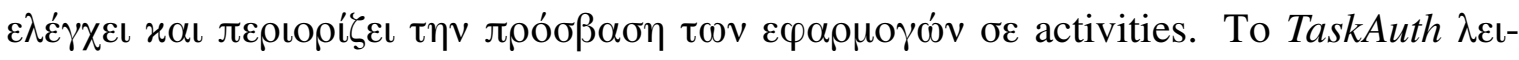

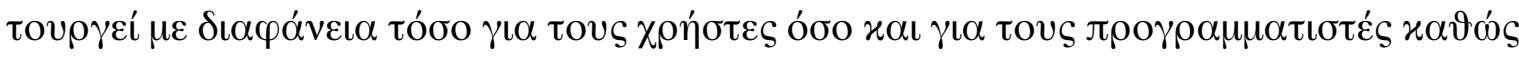

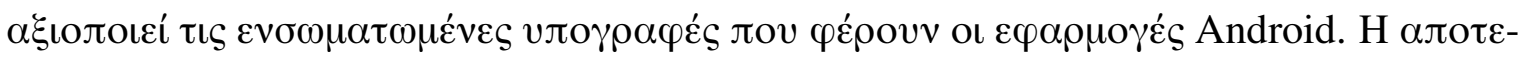

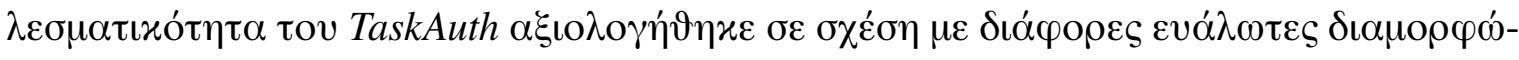

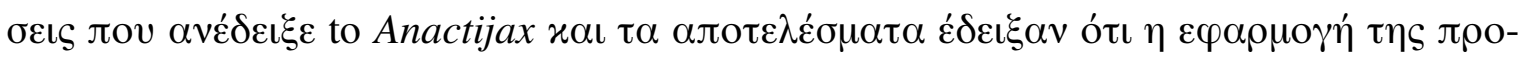

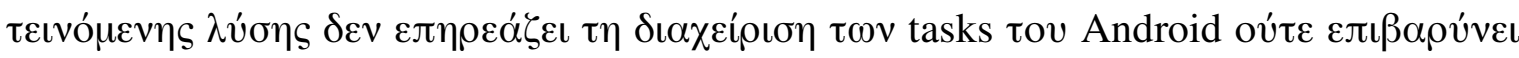

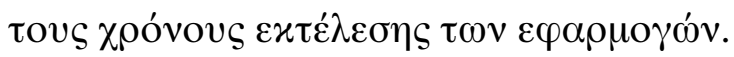

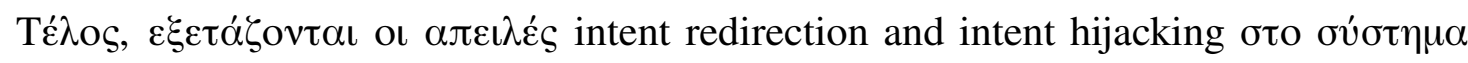

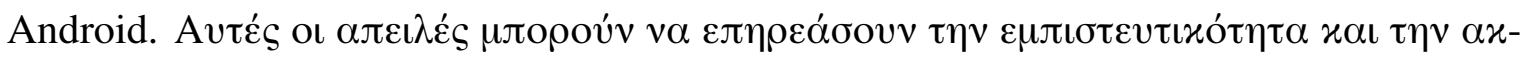

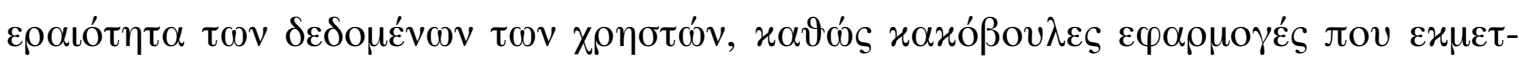

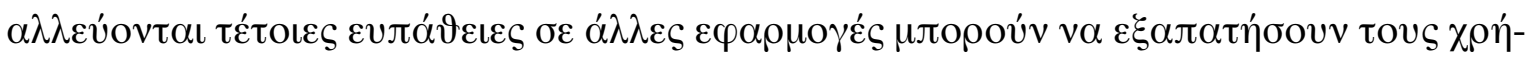

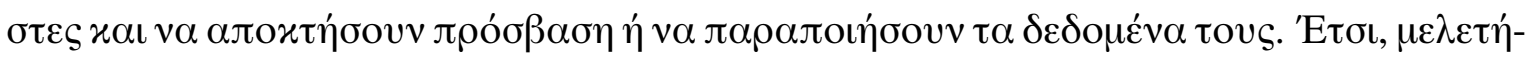

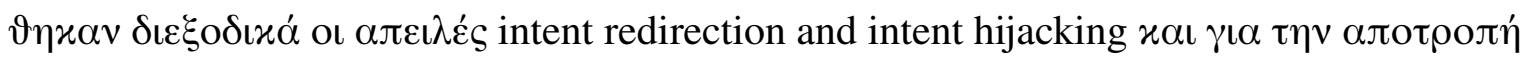

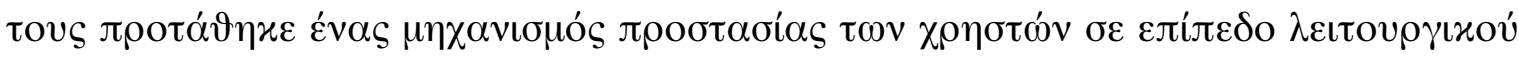

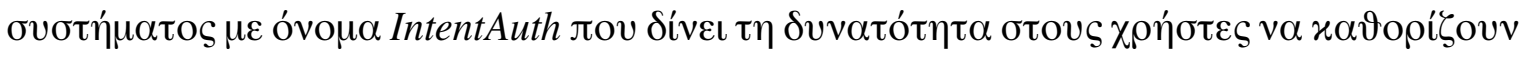

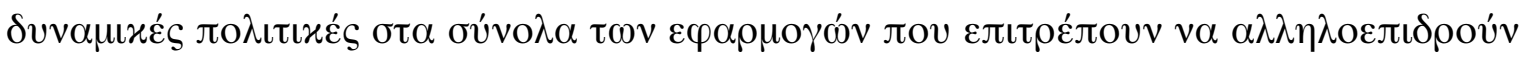

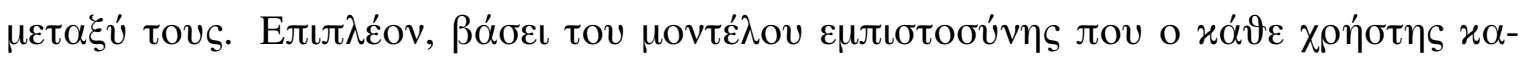

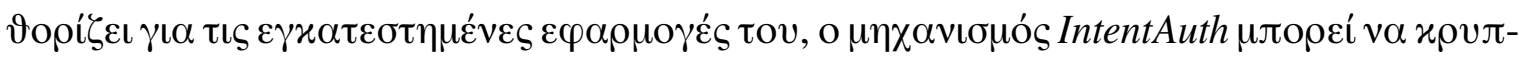

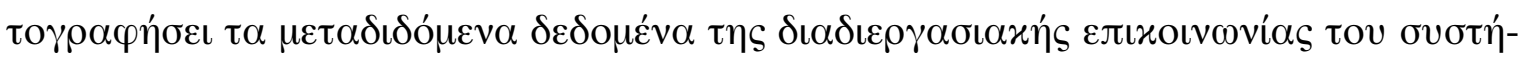

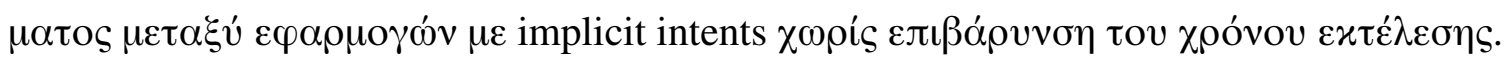

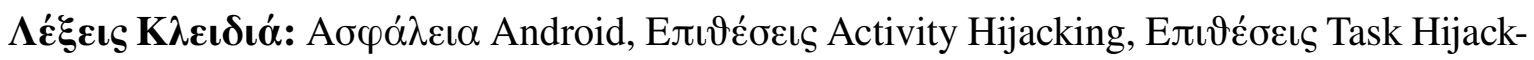

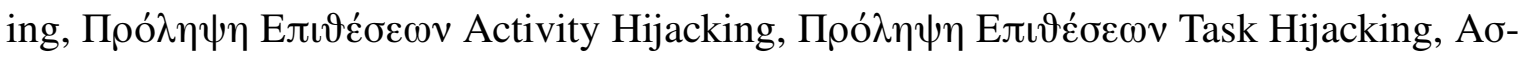

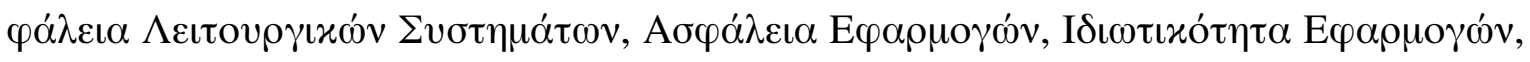

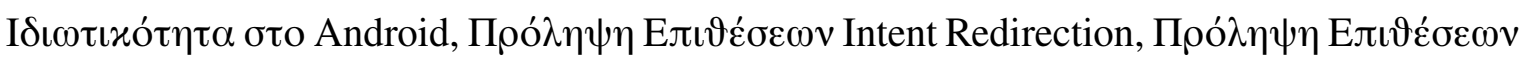

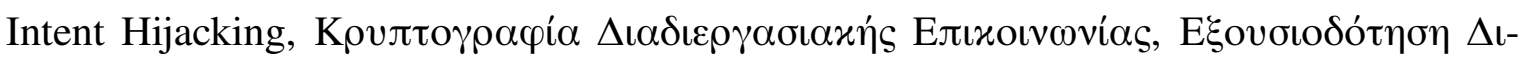




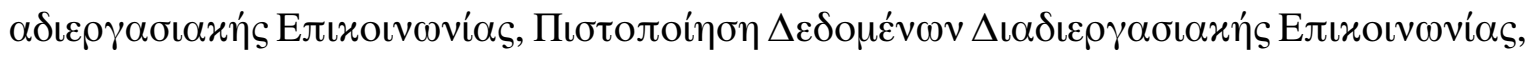
Android Permissions Map, Reflection Technique, $\Delta v v \alpha \mu \iota x \eta ́$ Avó $\lambda v \sigma \eta$ 



\section{Contents}

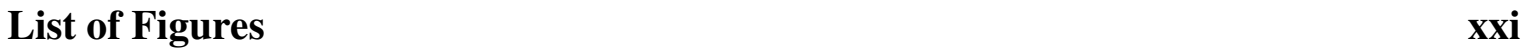

$\begin{array}{lc}\text { List of Tables } & \text { xxiii }\end{array}$

$\begin{array}{ll}\text { List of Algorithms } & \mathbf{x x v}\end{array}$

$\begin{array}{ll}\text { Abbreviations and Acronyms } & \text { xxvii }\end{array}$

1 Introduction 1

2 Contribution $\quad 7$

3 Android Overview 13

3.1 Android Framework Internals . . . . . . . . . . . . . . . . . . 13

3.1.1 Android Platform Architecture . . . . . . . . . . . . . . . 13

3.1.2 Android Application Components . . . . . . . . . . . . . . . 14

3.1.3 Android Inter-Process Communication . . . . . . . . . . . . 16

3.1.4 Android Task Handling . . . . . . . . . . . . . . . . . . . 16

3.2 Android Security Model _. . . . . . . . . . . . . . . . . 18

3.2.1 System Security _. . . . . . . . . . . . . . . 20

3.2 .2 Application Security . . . . . . . . . . . . . . . . 21

3.2.3 Exploit Prevention . . . . . . . . . . . . . . 22

4 Android Permission Model 2

4.1 System Level Permission Enforcement . . . . . . . . . . . . . . . . . . . 25

4.2 Android Application Permission Acquisition . . . . . . . . . . . . . . . . . . . . . . . . . . . . . 26

4.3 Threats of Android Permission Model . . . . . . . . . . . . . . . . 26

4.3.1 Permission Re-Delegation . . . . . . . . . . . . . 26

4.3.2 Over-Privileged Android Applications . . . . . . . . . . . . 28 
4.4 Android Permission Model Mapping . . . . . . . . . . . . . . . . . . . 28

4.4.1 Dynamic Analysis Based Approaches . . . . . . . . . . . . . . . 29

4.4.2 Static Analysis Based Approaches . . . . . . . . . . . . . 35

4.4.3 Experimental Evaluation . . . . . . . . . . . . 37

5 Android Task Management 43

5.1 Threats of Android Task Management . . . . . . . . . . . . . . 43

5.2 Android Task and Activity Hijacking Attacks . . . . . . . . . . . . 45

5.2.1 Exploitation and Detection Mechanisms . . . . . . . . . . . 45

5.2 .2 Prevention Mechanisms . . . . . . . . . . . . . 50

5.2 .3 Empirical Analysis . . . . . . . . . . . . . . 51

6 Android Intent Handling 57

6.1 Threats of Android Inter-Process Communication . . . . . . . . . . . 57

6.1 .1 Malicious Activity Launch and Intent Hijacking . . . . . . . . . . 59

6.1 .2 Intent Redirection . . . . . . . . . . . . . . . . . . 60

6.2 Enhancement Mechanisms for Android Inter-Process Communication . . . 61

6.2.1 Static \& Dynamic Analysis Detection . . . . . . . . . . . . . 61

6.2 .2 Runtime Protection . . . . . . . . . . . . . . 62

$\begin{array}{lll}7 & \text { Conclusions } & 67\end{array}$

Bibliography

Appendix A Intents Usage Examples 79

A.1 Implicit Intents . . . . . . . . . . . . . . . . . . . 79

A.2 Explicit Intents . . . . . . . . . . . . . . . . . . . . . 79

Appendix B Anactijax Generated Use Cases Examples $\quad 81$

B.1 Activity Injection Against any Benign Application . . . . . . . . . . . . . . 81

B.2 Activity Injection Against Vulnerable Benign Application's Tasks . . . . . 82

$\begin{array}{lr}\text { Index } & 83\end{array}$ 


\section{List of Figures}

3.1 Android Operating System Architecture . . . . . . . . . . . . . . . 15

3.2 Android Application Core Components and Fundamental Communication Channels Among Them. . . . . . . . . . . . . . . . . . . . . . 17

3.3 Android (Version 9) Activity Initialization and Task Handling. . . . . . . . 23

4.1 Android (Version 7) Permission Enforcement Control Point Example. . . . 27

4.2 Dypermin's Design for Permission Map Compilation . . . . . . . . . . . 30

4.3 Total number of methods with at least one permission as identified by Dypermin in comparison with Android software development kit source code and its online counterpart documentation. . . . . . . . . . . . . . . 40

4.4 A snapshot of Dypermin's time performance per method on Android Version

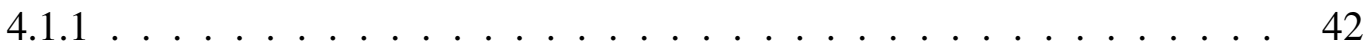

4.5 A snapshot of Dypermin's time performance per method on Android Version $6.0 \ldots \ldots \ldots \ldots \ldots$. . . . . . . . . . . . . . . . . . . .

5.1 Hijacking Execution Flows. . . . . . . . . . . . . . . . . . . . 45

5.2 High-Level Design of Anactijax for Task and Activity Injection Related Activity Attributes and Intent Flag Exfiltration. . . . . . . . . . . . . . 49

5.3 High-Level Design of Anactijax for Automated Hijacking Application Generation Against Real-World Applications. . . . . . . . . . . . . . . . . . 49

5.4 TaskAuth High Level Design. . . . . . . . . . . . . . . . . . . . . . . . 51

5.5 Overhead Introduced by TaskAuth to the (Default Exported) Main Activity . 53

5.6 Overhead Introduced by TaskAuth for Vulnerable Applications . . . . . . . 54

5.7 Overhead Introduced by TaskAuth For Each Real-World Application Used in the Evaluation (Total Two Hundred Applications) . . . . . . . . . . . . . . 54

6.1 Android (Version 11) Activity Handling. . . . . . . . . . . . . . . . . . 58

6.2 Intent Related Threats in Android Platform. . . . . . . . . . . . . . . . . 60

6.3 IntentAuth User-Defined Policies via Android (Version 11) Settings Application. 65 
6.4 IntentAuth High-Level Overview. . . . . . . . . . . . . . . . 65 


\section{List of Tables}

2.1 Technical Contribution of the Research Work Included in this Thesis. . . . 10

2.2 List of Publications Directly Related to the Work of this Thesis . . . . . . . 10

2.3 Contribution on Other Related Fields. . . . . . . . . . . . . . . . . . . 11

3.1 Primary Android Security Mechanisms and Their Enforcement Point. . . . 19

4.1 Method Comparison for Android Permission Mapping Generation . . . . . 37

4.2 Protected API methods coverage comparison between Dypermin and Android's available sources for Android version 4.1.1. . . . . . . . . . . . 39

4.3 Protected API methods coverage comparison between Dypermin and Android's available sources for Android version 6.0 . . . . . . . . . . . . . 41

5.1 Task and Activity Hijacking Related Works Comparison. . . . . . . . . . . 44

5.2 Anactijax Has Successfully Identified Hijacking Combinations of Activity Attributes and Intent Flags. . . . . . . . . . . . . . . . . 55

5.3 Execution Prevention of the Identified Activity Hijacking Use Cases of Anactijax after the Enforcement of TaskAuth . . . . . . . . . . . . . 56

6.1 Android Inter-Process Related Threat Prevention Mechanisms Comparison. 



\section{List of Algorithms}

4.1 Code Generated by Dypermin For registerNetworkAgent (. . .) Private API Method. . . . . . . . . . . . . . . . . . . . . . 32

A.1 Declaration of Activity Initiated by Implicit Intent. . . . . . . . . . . . 79

A.2 Invocation of an Activity with an Implicit Intent. . . . . . . . . . . . . 79

A.3 Declaration of Activity Initiated by Explicit Intent. . . . . . . . . . 79

A.4 Invocation of an Activity with an Explicit Intent. . . . . . . . . . . . . 80

B.1 Example of Manifest File Generated by Anactijax for Malicious Activity Injection Against a Default Benign Application. . . . . . . . . . . . . . 81

B.2 Example of Manifest File Generated by Anactijax for Default Vulnerable Benign Application. . . . . . . . . . . . . . . . . . . . . . . 81

B.3 Example of Manifest File Generated by Anactijax for Malicious Activity Injection Against a Vulnerable Benign Application with Explicitly Defined Tasks. . . . . . . . . . . . . . . . . . . 82

B.4 Example of Code Generated by Anactijax for Malicious Activity Injection Against a Vulnerable Benign Application with Explicitly Defined Tasks. . .

B.5 Example of Manifest File Generated by Anactijax for Vulnerable Benign Application with Explicitly Defined Tasks. . . . . . . . . . . . . . 82 



\section{Abbreviations and Acronyms}

$\begin{array}{ll}\text { ADB } & \text { Android Debug Bridge } \\ \text { AOSP } & \text { Android Open Source Project } \\ \text { AOT } & \text { Ahead-Of-Time } \\ \text { API } & \text { Application Programming Interface } \\ \text { APK } & \text { AndroidPacKage } \\ \text { APP } & \text { Application } \\ \text { ART } & \text { Android Runtime Environment } \\ \text { ASLR } & \text { Address Space Layout Randomization } \\ \text { ATMS } & \text { Activity Task Manager Service } \\ \text { CA } & \text { Certificate Authority } \\ \text { CFG } & \text { Control-Flow Graph } \\ \text { CPU } & \text { Central Processing Unit } \\ \text { DAC } & \text { Discretionary Access Control } \\ \text { DEX } & \text { Dalvik Executable } \\ \text { DVM } & \text { Dalvik Virtual Machine } \\ \text { FDE } & \text { File Based Encryption } \\ & \end{array}$




\begin{tabular}{|c|c|}
\hline HAL & Hardware Abstraction Layer \\
\hline HSM & Hardware Security Module \\
\hline ICC & Inter-Component Communication \\
\hline IMEI & International Mobile Equipment Identity \\
\hline iOS & iPhone Operating System \\
\hline IoT & Internet-of-Things \\
\hline IPC & Inter-Process Communication \\
\hline ITS & Intelligent Transportation Systems \\
\hline JIT & Just-in-Time \\
\hline JNI & Java Native Interface \\
\hline JVM & Java Virtual Machine \\
\hline MAC & Mandatory Access Control \\
\hline NDK & Native Development Kit \\
\hline NX & No eXecute \\
\hline OEM & Original Equipment Manufacturers \\
\hline OHA & Open Handset Alliance \\
\hline OS & Operating System \\
\hline PKI & Public Key Infrastructure \\
\hline RAM & Random-Access Memory \\
\hline RPC & Remote Procedure Calls \\
\hline SDK & Software Development Kit \\
\hline SMS & Short Message Service \\
\hline SoC & System on Chip \\
\hline $\mathrm{TE}$ & Trusted Execution Environment \\
\hline
\end{tabular}


UI User-Interface

UID User Identifier

URI Uniform Resource Identifier

VPN Virtual Private Network

XML eXtensible Markup Language 



\section{Chapter 1}

\section{Introduction}

Nowadays, mobile phones are not only used for making phone calls but they also support our digital interactions with different types of services i.e., banking, traveling, etc., through the corresponding mobile applications (apps). In the mobile world, currently, Android is considered the dominant operating system $(\mathrm{OS})^{1}$, with more than three million available applications for downloading (only) from Google play ${ }^{2}$. In fact, as Android end users basis is constantly expanding, more and more malicious software (malware) targets their devices for fun and profit ${ }^{3,4}$.

One pillar of Android's security is the process isolation at the kernel level, so that malevolent applications and services do not to affect the reliability of other services/applications or even of the device itself. Furthermore, it introduces an access control model, at application layer, for restricting access to "sensitive" resources (camera, location data, network, to mention a few) that could affect user's privacy or cause a security incident. Specifically, access to any sensitive resource is granted through a protected Application Programming Interface (API) method. An application in order to use a protected API method it must first declare the corresponding permissions in its manifest file, and request it also at runtime if it is executed on Android latest versions, otherwise a security exception is raised. In any case, users should give their consent for the permissions requested by the application either during the first time that a protected API method is invoked or during installation process, depending on the Android version. A more detailed analysis of the Android security model and the evolution of its permissions subsystem can be found in $[38,100]$.

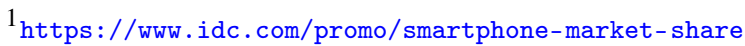

2 https://www.statista.com/statistics/266210/number-of-available-applications-in-the-google-play-store/

3 https://www.mcafee.com/enterprise/en-us/assets/reports/rp-mobile-threat-report-2019.pdf

${ }^{4}$ https://www.mcafee.com/content/dam/consumer/en-us/docs/2020-Mobile-Threat-Report.pdf
} 
Programmers get information on the correlation between permissions and protected API methods through Android's documentation. To this direction, an issue that attracts the attention of researchers, developers and Android enthusiasts is the question "What is the exact correlation between Android Software Development Kit (SDK) API methods and permissions?". This is caused by the fact that (a) documentation may accidentally lack information, and (b) Android has hidden and internal API methods that are not directly accessible at the application layer since they are not included in the Development SDK. Though the latter cannot be directly accessed there are several publicly available sources ${ }^{5}$ that give guidelines on how to gain access to such resources [58]. So eventually, programmers can gain access to these hidden API methods.

At this point it should be stressed that an accurate correlation between permissions and API methods is of high importance, as this correlation is utilized for malware detection [23] and other misconfigurations (i.e., over-privileges [44]) identification. Today there are attempts, such as Stowaway [41], PScout [24], Axplorer [25] and Bartel et al. [26], to compile the correlation of API methods - permissions that extend Android's documentation. However, these approaches are bounded to specific Android versions and also require access to the underlying OS source code.

Moreover, as the Android OS evolves and in order to improve end-users' experiences, it proceeds with various modifications to the underlying subsystems. For instance, as already mentioned, permissions are enabled dynamically on the latest versions of Android, while from version 6.0 backwards they were granted statically. In addition, some API methods are deprecated, while other are introduced to support additional functionalities. So it is evident that these types of changes not only affect the API methods and permission mapping but also introduce inconsistencies in it among different Android versions according to [100]. Thus even other solutions, such as DPSpec [29], that exclusively rely on annotations (e.g., of documentation) cannot provide a complete coverage for the Android permission mapping.

The research work of this thesis [65] has elaborated on the developments of Android's API methods - permissions mapping, by proposing a framework, called Dypermin, capable of generating the permission map in a transparent way without requiring access to the OS source code and without generating false positive alarms. Dypermin automatically invokes Android's publicly accessible and hidden API methods in order to intentionally raise runtime security exceptions and thus decide whether or not a permission dependency exists.

More specifically, Dypermin relies on the simple observation that the Android OS raises a security exception if a protected API method is invoked without the appropriate permissions being defined in the application's manifest file. Dypermin, in order to identify and report the

\footnotetext{
$5_{\text {https: //github.com/anggrayudi/android-hidden-api }}$
} 
permissions for every available API method, builds a single application that is automatically invoked after installation.

Dypermin achieves to extract all available API methods since it is provided through the SDK and thus it does not require any modifications to the underlying OS. Furthermore, it validates its finding as it relies on runtime information. Dypermin was evaluated with some well-known classes for different Android versions and its results are compared with both Android public and SDK source documentation as well as with the results of other related proposed methods. Currently, Dypermin does not provide a full mapping since it takes a substantial amount of time but it has been proved that it can accurately identify the API methods - permission map and deduce whether the available documentation is missing a relation between an API method and a permission.

Various types of malware [40], such as trojan, backdoors etc., target mobile devices. Even though various protection techniques $[54,62,72,98]$ have been introduced in order to enhance their protection level and minimise the attack surface for Android platform, malicious entities do not only find new approaches to by pass the deployed countermeasures, but they also manage to launch their attacks silently $[39,97]$. Researches have shown $[84,74]$ that attackers can bypass security checks and that malicious software can be published even in trusted sources.

In fact, some of Android security flaws are because of its divergent characteristics, something which is not the case for typical OS distributions. For instance, Android allows applications: (a) to monitor end users devices' sensors by granting the appropriate permission(s), (b) to interact directly with other applications, services and components through inter-process communication (IPC), and (c) combine activities from different applications into a certain task. Unlike other operating systems, an Android task is a combination of activities that end users interact with in order to perform a certain job; note that activities are the core element of Android's User Interfaces (UI) and are organized in tasks. Even though this functionality enables and improves end users experience on seamless transfer among different activities and applications, it may turn them vulnerable to activity injection $[81,89,79,80,48]$ and task hijacking attacks $[55,48,93,61,92]$. Such attacks aim to deceive end users, by masquerading silently User Interfaces (UI), and steal credentials or other sensitive data provided to the targeted application.

Currently, various researchers have attempted to address task [55, 48, 93, 61, 92] and activity $[81,89,79,80]$ hijacking vulnerabilities, through various different approaches. While the effectiveness of these researches is not questionable, some of them have become obsolete $[81,89]$ due to Android framework's revisions, others require device 'rooting' [80] to enforce controls, others demand end users' intervention [93] assuming that their capable of 
recognizing malicious activities, others suffers from well-known limitations of static analysis in Android applications (for instance: disregard of implicit intents invocations) [55, 48]. Orthogonal solutions, such as [61], propose the deployment of additional static and dynamic analysis controls at vetting process, whereas authors in [92] introduce a system level mechanism, by modifying the underlying OS, in order to inform end users for possible activity hijacking attacks. In [48] a reformation of the current Android application development framework is required for introducing a signature based mechanism to protect Android applications against task hijacking attacks.

In an attempt to complement and improve the existing security provisions, research work presented in this thesis [66] aims to enhance the security of Android's task handling mechanism. The preconditions of task injection and activity hijacking attacks have been studied by introducing an open source tool called Anactijax that is capable of automatically generating activity injection test cases for assessing whether an application is vulnerable or not to such type of attacks. Moreover, they presented a system level authorization mechanism named TaskAuth that minimises the attack surface of Android's task handling mechanism by correlating the origin of interacting applications through their carrying signatures. TaskAuth controls the access among applications' activities using dynamic predefined policies i.e., allow only vendor application or applications by the same developer to acquire a task from another application to their process. TaskAuth effectiveness has been evaluated in terms of possible implication on OS functionality and the overhead it introduces i.e., how end user's experience is affected. The experiments performed highlight that TaskAuth does not affect Android's task handling core mechanism functionality, while the execution overhead introduced is considered negligible as it is not more than $4.3 \mathrm{~ms}$ in average.

Finally, another class of threats against the Android platform regards attacks against it's Inter-Process Communication (IPC) mechanism. As already mentioned, Android has divergent characteristics that are not found in typical OS distributions, such as the InterProcess Communication (IPC) or, similarly, the Inter-Component Communication (ICC) mechanism similar to the "client-server" model. Due to its implementation peculiarities, Android's Inter-Process communication mechanism needs to employ protection measures against Intent Redirection [85, 37, 88] and Intent Hijacking [31, 95] attacks. The former category concerns cases where malicious applications leverage publicly available exported components (i.e., Activities) and transfer data to other components that perform sensitive operations upon them. The latest threat concerns malicious applications that define Intent filters to intercept data transmitted by a legitimate application.

Currently, various research works attempt to address these security flaws using different approaches ranging from (a) static analysis detection tools [85, 37] to (b) system-level en- 
hancements to prevent such attacks $[88,95]$ and minimize the OS's attack surface. While the effectiveness of these researches is not questionable, some of them suffer [85, 37] from the common limitations of static analysis approaches, while others that require the modification of the underlying OS, cannot be re-configured [88] dynamically, or expose intents beyond the interaction processes [95].

The research work of this thesis [68] complements and improves existing security provisions, by enhancing Android's IPC mechanism security. In particular, the security flaws associated with intent activity launch, intent hijacking and redirection have been thoroughly studied and a novel and practical open source system-level mechanism, namely IntentAuth (Intent Authentication), has been proposed enabling secure communication between applications and thus protecting users against such attacks. In fact, IntentAuth's goal is to minimize, if not eliminate, benign applications' attack surface by authenticating applications' communicating components through asymmetric key cryptography, and securing data exchange through symmetric key cryptography, employing user defined dynamic policies. In this way, users can control each applications' inter processes communication access transparently.

The rest of this thesis is structured as follows: Chapter 2 provides an overview and the main contributions of the conducted research. Chapter 3 provides an overview of the Android Operating System's core components, emphasizing on its security architecture. Chapter 4 briefly describes the Android Framework's permission model, the related threats and the methodologies proposed in the literature for the accurate construction of the Android's permission map. Chapter 5 describes the platform's task handling mechanism, the methodologies proposed in the literature for the investigation of the attack surface, and the mitigation mechanisms for eliminating the identified vulnerabilities. Chapter 6 presents the Android's Inter-Process Communication model with intents, its attack surface, and the mitigation mechanisms, as resulted from the relevant literature review, for addressing the identified threats. Finally, Chapter 7 concludes the thesis and provides remarks for future work. 



\section{Chapter 2}

\section{Contribution}

Android is a custom Linux-based operating system. The primary running environment is the Dalvik Virtual Machine (DVM) similar to the Java Virtual Machine (JVM) in the initial versions or the Android Runtime Environment (ART) in the newer versions. Due to its architectural uniqueness, the Android Operating System (OS) is facing new types of threats and attacks. These new threats and attacks mainly concern the Android framework which adopts a "Client-Server" model for application interaction (Inter-Process Communication IPC) or, similarly, Inter-Component Communication - ICC). Additionally, threats arise due to partial access control to sensitive system resources and actions from malicious applications.

This thesis, as depicted in Table 2.1 and Table 2.2, is focusing on how threats and attacks against the Android platform can be mitigated. More specifically automated tools for a) evaluating the platform's security mechanisms and b) exploiting vulnerabilities have been developed, as well as mechanisms for preventing attacks have been proposed.

Chapter 3 presents the Android operating system's architecture and all the technical features that govern it, along with all the fundamental security mechanisms that protect it at both system and application level.

Chapter 4 focuses on one of the most important security and privacy mechanism of the Android platform, which is the application permission model. According to this model, an Android application must explicitly define permissions when using sensitive API (Application Programming Interface) framework methods. The user should accept it for an application to gain access to sensitive system resources (such as geographical location or access to users' messages) or perform actions such as making phone calls or sending SMS. Understandably, there is a strong relationship between the Android Framework methods and the permissions that protect them. However, this correlation is not given as there are multiple control points of permissions throughout the Android Framework. Although Google systematically reports in the Android API documentation the permissions a method needs, deficiencies have been 
observed. The documentation descriptions confuse developers several times, leading to misconfigurations or misuse of permissions, which is a significant security risk for applications. Taking into account the above context and assumptions, a methodology has been developed to strictly correlate Android permissions with the methods they protect by extending the existing literature that mainly suffers from false positives due to static analysis methods limitations or operating system modifications. This methodology does not require the modification of the operating system and does not suffer from false positives, as it utilizes techniques provided by the Android Framework to create automated applications, execute them and examine if security exceptions were caused during their execution [65]. With this approach, the required permission for the proper invocation of methods can be extracted from the exception traces. The result of this work was the Dypermin ${ }^{1}$ tool.

Chapter 5 investigates and redefines the Task and Activity Hijacking attacks [66]. A mechanism named Anactijax ${ }^{2,3}$ has been developed to identify possible combinations of Activity attributes and Intent Flags that a malicious application can use to exploit design weaknesses of the Android Framework or applications in order to deceive users or intercept their data. To prevent this attack, a system-level mechanism was developed, named TaskAuth ${ }^{4}$ (Task Authorization). This mechanism checks the ownership of the applications involved, based on the signatures and certificates they carry from their developers. For TaskAuth, the distinction between the Activity Hijacking attack and the Activity Injection technique is related to the ownership of the applications involved. More specifically, if an application tries to inject an Activity into a task or process of another application, it is checked whether the two applications belong to the same developer. If the verification fails, Activity hijacking is dynamically prevented while using this technique it is allowed only for system applications or between applications belonging to the same developer. The above-mentioned mechanism extends the capabilities of other methods proposed in the literature and at the same time it addresses some of their practical limitations, as it neither requires an unsafe device configuration to operate nor it involves users or developers creating complex policies that allow or prevent Activity Injection between applications. Finally, TaskAuth is completely transparent to the users as it does not prevent applications that use Activity Injection techniques from running without meeting the specifications it has set.

Chapter 6, focuses on the threats of Inter-Process Communication of the Android operating system and, more specifically, on Intent Redirection and Intent Hijacking [68]. Intents are the means of communication between processes on the Android platform. A component

\footnotetext{
${ }^{1}$ Dypermin Implementation: https ://gitlab.ds .unipi.gr/systems-security-laboratory/dypermin

${ }^{2}$ Anactijax Implementation: https://gitlab.ds .unipi.gr/systems-security-laboratory/anactijax

${ }^{3}$ Anactijax Results: https://gitlab.ds.unipi.gr/systems-security-laboratory/anactijax-results

${ }^{4}$ TaskAuth Implementation: https://gitlab.ds .unipi.gr/systems-security-laboratory/taskauth
} 
to communicate and exchange data with another component that belongs to the same or another application uses Intents. The Intent Redirection threat occurs when a malicious application sends data through intents to a benign application in order for the latter to process it and perform an action on behalf of the malware based on the received data. In this threat model, the benign application performs a sensitive and not a malicious action. However, receiving and using data from an untrusted application may turn the sensitive action into a malicious one. Another threat against Intents are the Hijacking attacks, where a malicious application intercepts the intents intended for a benign application. In order to prevent the above threats, a system-level security mechanism has been developed offering to users the ability to setup dynamic policies specifying the applications that are allowed to exchange data and communicate with each other. The developed mechanism is called IntentAuth ${ }^{5}$ (Intent Authentication) and in addition to the creation of dynamic policies it supports authentication of transmitted data among processes. Hence, based on the user-defined trust model, IntentAuth, can impose end-to-end (application to application) cryptography and signature verification in order to protect the data transmitted between processes in the service-oriented Framework of Android. To achieve that, IntentAuth takes advantage of existing operating system mechanisms, such as SELinux and cryptographic keys, generated by Trusted Execution Environment (TEE) or Hardware Security Module (HSM). This enables IntentAuth to ensure the authenticity, integrity, and confidentiality of both transmitted data and the cryptographic keys of the interacting applications.

Finally, Chapter 7 concludes the research carried out and provides remarks for future work and improvements regarding the enhancement of the Android Inter-Process Communication scheme.

\footnotetext{
${ }^{5}$ IntentAuth Implementation: https://gitlab.ds.unipi.gr/systems-security-laboratory/intentauth
} 


\begin{tabular}{|c|c|c|c|c|c|c|}
\hline \multirow[t]{2}{*}{ Solution } & \multicolumn{3}{|c|}{ Type } & \multirow[t]{2}{*}{ Category } & \multirow[t]{2}{*}{ Enforcement Layer } & \multirow[t]{2}{*}{ Availability } \\
\hline & Prevention & $\begin{array}{c}\text { Detection } \\
\text { Recognition }\end{array}$ & Exploitation & & & \\
\hline Dypermin [65] & No & Yes & No & Permission Map & Application & Yes \\
\hline \multirow{2}{*}{ Anactijax [66] } & \multirow{2}{*}{ No } & \multirow{2}{*}{ Yes } & \multirow{2}{*}{ Yes } & Task Hijacking & \multirow{2}{*}{ Application } & \multirow{2}{*}{ Yes } \\
\hline & & & & Activity Hijacking & & \\
\hline \multirow{2}{*}{ TaskAuth [66] } & \multirow{2}{*}{ Yes } & \multirow{2}{*}{ No } & \multirow{2}{*}{ No } & Task Hijacking & \multirow{2}{*}{ Operating System } & \multirow{2}{*}{ Yes } \\
\hline & & & & Activity Hijacking & & \\
\hline \multirow{3}{*}{ IntentAuth [68] } & \multirow{3}{*}{ Yes } & \multirow{3}{*}{ No } & \multirow{3}{*}{ No } & Intent Hijacking & \multirow{3}{*}{ Operating System } & \multirow{3}{*}{ Yes } \\
\hline & & & & $\begin{array}{l}\text { Malicious Activity } \\
\text { Launch }\end{array}$ & & \\
\hline & & & & Intent Redirection & & \\
\hline
\end{tabular}

Table 2.1 Technical Contribution of the Research Work Included in this Thesis.

\begin{tabular}{|l|l|c|}
\hline \multicolumn{1}{|c|}{ Research } & Authors & Topic \\
\hline $\begin{array}{l}\text { Dypermin: Dynamic Permission Mining } \\
\text { Framework for Android Platform, Com- } \\
\text { puters \& Security [65] }\end{array}$ & $\begin{array}{l}\text { Christos Lyvas, Costas Lambrinoudakis, } \\
\text { and Dimitris Geneiatakis }\end{array}$ & $\begin{array}{c}\text { Android Operating System Security and } \\
\text { Privacy }\end{array}$ \\
\hline $\begin{array}{l}\text { On Android's Activity Hijacking Preven- } \\
\text { tion, Computers \& Security [66] }\end{array}$ & $\begin{array}{l}\text { Christos Lyvas, Costas Lambrinoudakis, } \\
\text { and Dimitris Geneiatakis }\end{array}$ & $\begin{array}{c}\text { Android Operating System Security and } \\
\text { Privacy }\end{array}$ \\
\hline $\begin{array}{l}\text { IntentAuth: Securing Android's Intent } \\
\text { Based Inter-Process Communication, In- } \\
\text { ternational Journal of Information Secu- } \\
\text { rity [68] }\end{array}$ & $\begin{array}{l}\text { Christos Lyvas, Costas Lambrinoudakis, } \\
\text { and Dimitris Geneiatakis }\end{array}$ & $\begin{array}{c}\text { Android Operating System Security and } \\
\text { Privacy }\end{array}$ \\
\hline
\end{tabular}

Table 2.2 List of Publications Directly Related to the Work of this Thesis

Even though this thesis focuses on the security of the Android operating system, in the course of the work research was also carried out in other related topics such as the security of the iOS Operating System, intrusion detection for virtual operating system environments, automated malware generation, and security on IoT environments, especially for Intelligent Transportation Systems (ITS) as Table 2.3 summarizes. The knowledge gained from the aforementioned related research activities provided useful ideas and techniques for this thesis. 


\begin{tabular}{|c|c|c|}
\hline Research & Authors & Topic \\
\hline $\begin{array}{l}\text { The Far Side of Mobile Application Inte- } \\
\text { grated. Development Environments [64] }\end{array}$ & $\begin{array}{l}\text { Christos Lyvas, Nikolaos Pitropakis, and } \\
\text { Costas Lambrinoudakis }\end{array}$ & iOS Application Security \\
\hline $\begin{array}{l}\text { [m]allotROPism: A Metamorphic En- } \\
\text { gine for Malicious Software. Variation } \\
\text { Development [67] }\end{array}$ & $\begin{array}{l}\text { Christos Lyvas, Christoforos Ntanto- } \\
\text { gian, and Christos Xenakis }\end{array}$ & Malicious Software Development \\
\hline $\begin{array}{l}\text { The Greater The Power, The More Dan- } \\
\text { gerous The Abuse: Facing Malicious. In- } \\
\text { siders in The Cloud [78] }\end{array}$ & $\begin{array}{l}\text { Nikolaos Pitropakis, Christos Lyvas, and } \\
\text { Costas Lambrinoudakis }\end{array}$ & $\begin{array}{l}\text { Intrusion Detection System for Virtual } \\
\text { Operating System Environments }\end{array}$ \\
\hline $\begin{array}{l}\text { Towards the Design of an Assurance } \\
\text { Framework for Increasing Security and } \\
\text { Privacy in Connected Vehicles [51] }\end{array}$ & $\begin{array}{l}\text { Christos Kalloniatis, Vasiliki Diaman- } \\
\text { topoulou, Konstantinos Kotis, Christos } \\
\text { Lyvas, KonstantinosMaliatsos, Matthieu } \\
\text { Gay, Athanasios Kanatas, and Costas } \\
\text { Lambrinoudakis }\end{array}$ & $\begin{array}{c}\text { Intelligent Transportation Systems } \\
\text { Security }\end{array}$ \\
\hline $\begin{array}{l}\text { Standardizing Security Evaluation Crite- } \\
\text { ria for Connected Vehicles: A Modular } \\
\text { Protection Profile [69] }\end{array}$ & $\begin{array}{l}\text { Konstantinos Maliatsos, Christos Ly- } \\
\text { vas, Panagiotis Pantazopoulos, Costas } \\
\text { Lambrinoudakis, Athanasios Kanatas, } \\
\text { Matthieu Gay, and Angelos Amditis }\end{array}$ & $\begin{array}{c}\text { Intelligent Transportation Systems } \\
\text { Security }\end{array}$ \\
\hline $\begin{array}{l}\text { Aligning the Concepts of Risk, Security } \\
\text { and Privacy Towards the Design of Se- } \\
\text { cure Intelligent Transport Systems [35] }\end{array}$ & $\begin{array}{l}\text { Vasiliki Diamantopoulou, Christos } \\
\text { Kalloniatis, Christos Lyvas, Kon- } \\
\text { stantinos Maliatsos, Matthieu Gay, } \\
\text { Athanasios Kanatas, and Costas Lambri- } \\
\text { noudakis }\end{array}$ & $\begin{array}{c}\text { Intelligent Transportation Systems } \\
\text { Security }\end{array}$ \\
\hline
\end{tabular}

Table 2.3 Contribution on Other Related Fields. 



\section{Chapter 3}

\section{Android Overview}

\subsection{Android Framework Internals}

Android is an open-source mobile operating system (OS) officially released in 2007 by Google and the Open Handset Alliance (OHA). Since then, Android has managed to be established as the dominant operating system for mobile and other Internet of Things (IoT) devices. This is mainly because of its open-source nature that enables Original Equipment Manufacturers (OEMs) to use Android as the core operating system of their devices.

\subsubsection{Android Platform Architecture}

The Android platform consists of several layers, as Figure 3.1 depicts. The operating system's baseline is the reformed Linux kernel [53] responsible for orchestrating and managing hardware via drivers, memory, processes, and the Inter-Process communication mechanism based on Binder. Apart from the Android kernel, in the baseline of its architecture, resides the Secure Element, which can be the Trusted Execution Environment (TEE) that is implemented upon the System on Chip (SoC) and Central Processing Unit (CPU) system-wide approach of ARM TrustZone [46] or a dedicated Hardware Security Module (HSM) that is responsible for several critical cryptographic operations. The implementation of the Trusted Execution Environment (TEE) for Android is provided by an isolated operating system called Trusty OS [22], which is secluded from the rest of the system by hardware and software. On top of the layers mentioned above resides the Hardware Abstraction Layer (HAL) that exposes standard programming interfaces for device hardware management to the higher-level layers. On top of HAL resides the Android Runtime Environment (ART) and all the supporting Native C/C++ Libraries. In the Android platform's initial releases, during the application's execution, the JIT (Just-in-Time) compiler was responsible for translating the Dalvik Executable (DEX) 
application format to machine code and executed it into multiple virtual machines (Dalvik Virtual Machine - DVM) similar to the Java Virtual Machine (JVM). Where the Dalvik Virtual Machine kernel (Zygote DVM) was responsible for creating separate, secure processes in shared memory, DVM was directly deployed as the execution environment for each application. Android Runtime Environment (ART) replaced the Dalvik Virtual Machine with the Ahead-Of-Time (AOT) compilation, where the transformation occurs at the installation phase of each application. However, the use of a virtual machine as an execution environment for each application still holds. Above the Android Runtime and native libraries reside the Android System Services that provide functionality exposed by application framework APIs (Application Programming Interfaces) that communicate with system services to access the underlying hardware. Application developers may interact mostly with two groups of services: a) the system (services such as window manager and package manager) and b) media (services involved in playing and recording media). These are the services that provide application interfaces as part of the Android framework. Besides these services, there are also native services similar to Linux daemons supporting these system services, such as netd, logcatd, etc. Another layer of the Android architecture is the Android Application Programming Interface (API), namely Android Framework, which offers all the functionalities and features of the Android operating system to the developers for building their applications. Finally, in the top layer of the architecture reside the Android applications divided into the pre-installed system applications that have been developed by vendors or OEMs for handling critical capabilities of the device (such as SMS, phone calls, etc.), and the user-installed applications that can be found and installed through several distribution channels such as the official Android application market called Google Play ${ }^{1}$ or other secondary sources such as Amazon Appstore $^{2}$ or Samsung Galaxy Store ${ }^{3}$.

\subsubsection{Android Application Components}

Android applications can be developed in Java, $\mathrm{C}++$, and Kotlin programming languages [1]. An AndroidPacKage (APK) archive is generated after the successful compilation of the source files that can be installed in the targeted Android device. The Java or Kotlin programming language are the recommended languages for developing Android applications over the standard Java Android SDK (Software Development Kit). At the same time, applications may contain native code as libraries with the aid of the Android Native Development Kit (NDK). Java Native Interface (JNI) is the framework that enables Java to call and be called by native appli-

\footnotetext{
$1_{\text {https://play.google.com/store }}$

2 https://www . amazon. com/mobile-apps/b?ie=UTF8\&node=2350149011

${ }^{3}$ https://www. samsung.com/global/galaxy/apps/galaxy-store/
} 


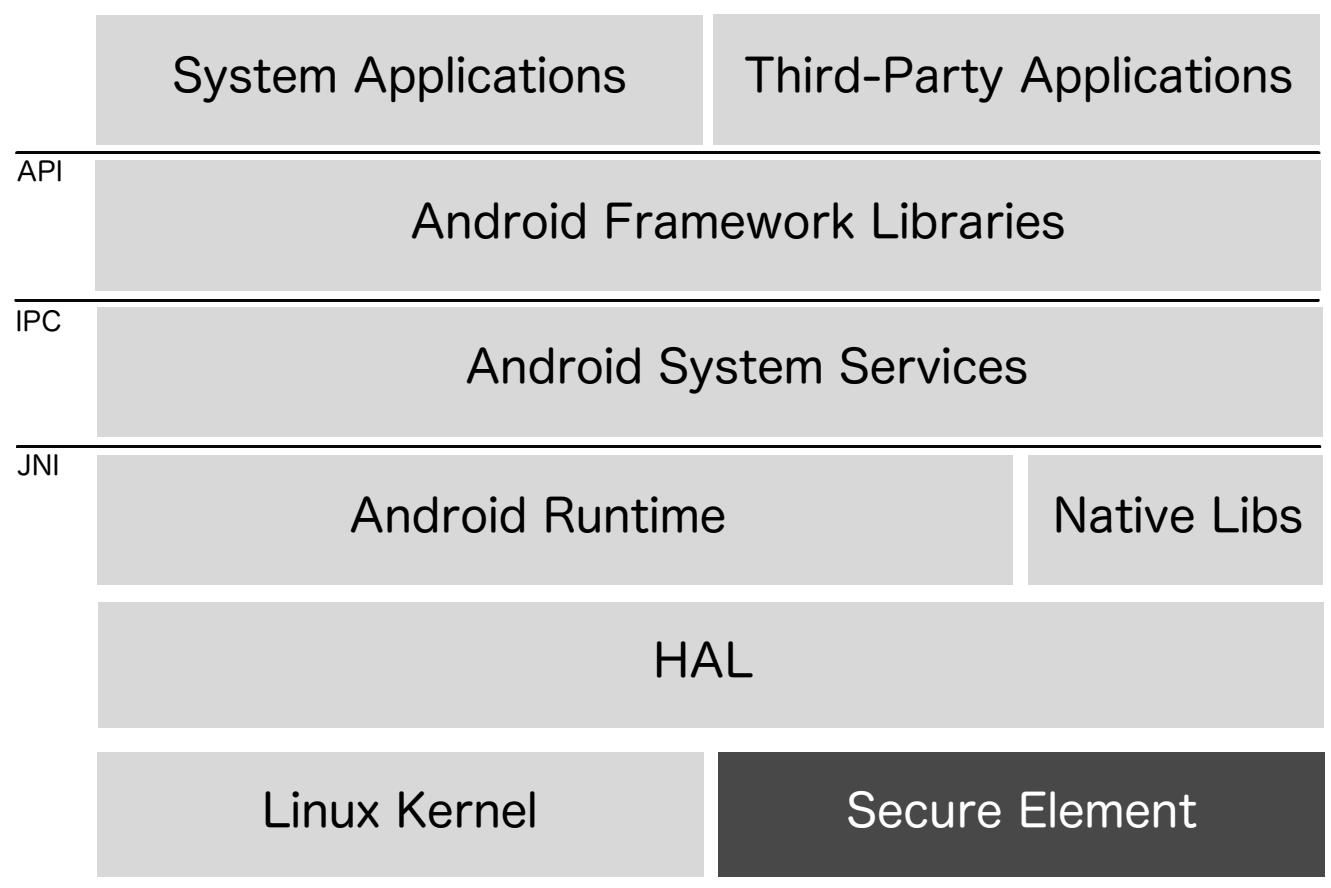

Figure 3.1 Android Operating System Architecture.

cations and libraries written in unmanaged code $(\mathrm{C} / \mathrm{C}++)$. An Android application, without loss of generality, may consist of one or more combinations of the following components:

- Activities comprise the (UI) of Android applications and enables end user interaction with the device.

- Broadcast Receivers allow the system to send events to applications outside the normal execution flow, and execute in the background to handle incoming events.

- Services run in the background, as well, to perform a variety of computational tasks on behalf of the application.

- Content Providers enable applications to store internal data or share them with other Android components.

These components as well as the required permission are declared in application's Manifest; otherwise, the application does not function properly. 


\subsubsection{Android Inter-Process Communication}

Android OS does not allow Android application components to exchange information directly with each other whenever it is needed. In general, Android OS relies on Inter-Process Communication (IPC) [52] or equivalently Inter-Component Communication (ICC) to enable the communication between different processes following a "server-client" model.

In fact, in Android OS, IPC is a mechanism IPC is a mechanism that enables one application to use a component of another application and synchronize their actions by using remote procedure calls (RPCs) $[83,28]$. In a nutshell, Android OS has two fundamental IPC mechanisms:

(a) the Binder [16], considered to be the core of Android's IPC mechanism, that allows app's processes to interact with other components and services at kernel level. So at low level, all communications between applications' components are established through the Binder IPC [52]. It consists of the Server, the Service Manager, the Client, and the Binder components. A client requests a service and the Server is the responsible process for providing that service. Simultaneously, the Service Manager handles the registered service [86], and

(b) the Intent [2], which provides a higher-level of abstraction that is executed over the Binder and is frequently deployed at the application layer for ICC communication [52]. Briefly, an Intent is a messaging object that carries data used to start an activity, communicates with services, or broadcasts messages [75]. In fact, intents can be used either explicitly or implicitly. In the former the application defines the target activity, service or broadcast receiver that will handle the intent, while on the latter the system or the user will determine which component should take care of it. Briefly, as Figure 3.2 depicts, an Intent is a messaging object that may carry data used to start an activity, communicate with services, or send broadcast messages [75].

Intents are divided into implicit and explicit. Explicit Intents (A.3) are used when the name of the class of target activity, service, or broadcast receiver that will handle the intent is defined (A.4). Implicit Intents (A.1) are used by the declaration of a general action to perform (A.2), rather than the class name's definition. At the same time, the system will determine the appropriate activity or broadcast receiver to handle the intent.

\subsubsection{Android Task Handling}

Android starts a new process [3], upon the initiation of an application's component (i.e., activities), with one thread of execution. In case that another application component starts, assuming that the application has been already initiated, it is executed under the same process sharing the same thread; meaning that all components (of the same application) are executed 


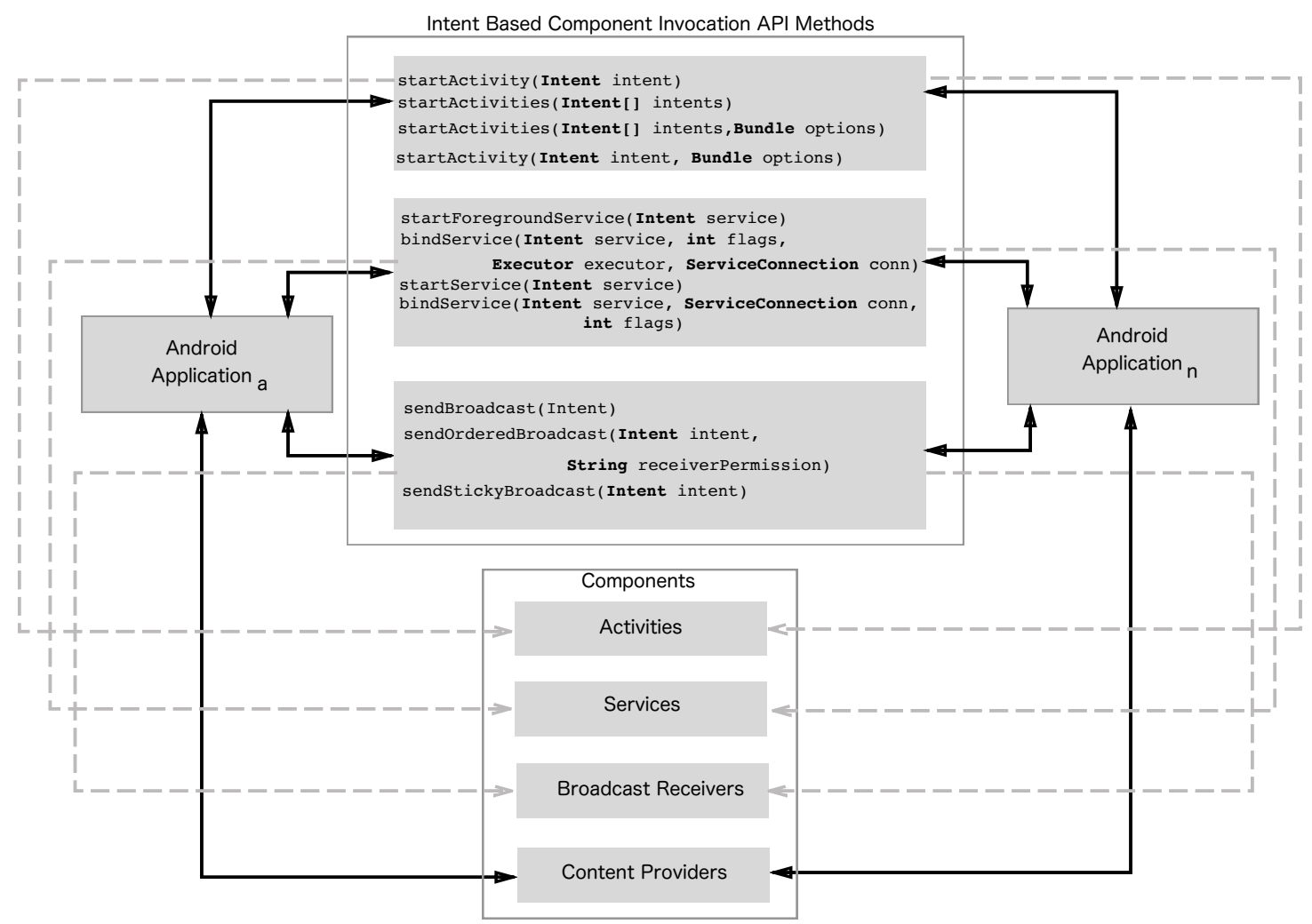

Figure 3.2 Android Application Core Components and Fundamental Communication Channels Among Them.

under the same process and thread, except if the application's developer has defined that specific components need to run under a separate process.

Android handles activities as a collection (of activities) named as task [4]. Usually, a task is limited within the context of an application, however, it may also contain activities from different applications [79]. Inside a task, activities are managed as a Last-in-FirstOut (LIFO) [80] data structure and support only push and pop functions. So whenever an application initiates a new activity ${ }^{4}$, the Android OS pushes the latter on the top of the stack, being in the foreground, while previous activities are pushed down in the stack without being executed. If end user presses the back button, the foreground activity is popped from the top of the stack and its execution is stopped, while the activity that is now on the top of the stack is restored. Figure 3.3 overviews activity initialization mechanism from the OS perspective.

\footnotetext{
${ }^{4}$ The initialization of an activity requires the invocation of the startActivity(...) method of the Activity class.
} 
In this context, the ActivityManagerService (AMS) $)^{5}$ class handles the life-cycle of all activities and gives to applications the capacity to communicate with it through the ActivityManager interface ${ }^{6}$. Thus for activities task management purposes the method startActivityAsUser ( . . ) is called to initialise a task which finally triggers the startActivityMayWait (. . . ) method, belonging to ActivityManagerService, and ActivityStarter ${ }^{7}$ classes correspondingly.

At this point, it should be noted that the startActivityMayWait (...) method is fundamental for the analysis as it contains all the information concerning the assignment of activities into tasks. Specifically, it manages the aInfo object of ActivityInfo class which holds all the information regarding application's activities i.e., permissions required for an activity to be executed, task affinity name, etc.Moreover, Android OS through its API allows (developers) to change activities' default association with a task. For instance, it might be desired the back stack to be cleared. This can be achieved by defining the appropriate activity attributes either in application's manifest or in its source code. Such attributes enable the activities to (a) assign an activity to a new task, (b) clear the current stack, and (c) relaunch an activity from the stack if already exists in it, while all the rest, on top of it in the stack, are destroyed.

\subsection{Android Security Model}

Android platform is a mobile operating system with several security mechanisms enforced in multiple layers, as presented in Table 3.1 shows.

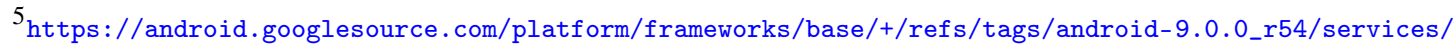
core/java/com/android/server/am/ActivityManagerService.java

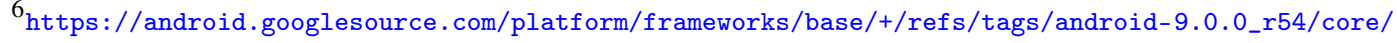
java/android/app/IActivityManager.aidl

7 https://android.googlesource.com/platform/frameworks/base/+/refs/tags/android-9.0.0_r54/services/ core/java/com/android/server/am/ActivityStarter.java
} 


\begin{tabular}{|c|c|c|}
\hline Security Mechanism & Android Version & Layer of Enforcement \\
\hline Application-Level Permissions & 1.0 & Android Framework \\
\hline Application Signing & 1.0 & Android Framework \\
\hline Discretionary Access Control (DAC) & 1.0 & Kernel \\
\hline No eXecute (NX) & 2.3 & Kernel \\
\hline Random Stack Canary & 4.0 & Kernel \\
\hline Address Space Layout Randomization (ASLR) & 4.0 & Kernel \\
\hline Mandatory Access Control (MAC) & 4.3 & Kernel \\
\hline Keystore System & 4.3 & TEE \\
\hline Verified Boot & 4.4 & Kernel \\
\hline \multirow[t]{2}{*}{ Full Disk Encryption (FDE) } & \multirow[t]{2}{*}{5.0} & TEE \\
\hline & & OS \\
\hline \multirow[t]{2}{*}{ Gatekeeper } & \multirow[t]{2}{*}{6.0} & TEE \\
\hline & & OS \\
\hline File Based Encryption (FBE) & 7.0 & OS \\
\hline Strongbox Keymaster System & 9.0 & HSM \\
\hline \multirow[t]{2}{*}{ Weaver } & \multirow[t]{2}{*}{9.0} & HSM \\
\hline & & OS \\
\hline
\end{tabular}

Table 3.1 Primary Android Security Mechanisms and Their Enforcement Point. 


\subsubsection{System Security}

Each application (as a UNIX-based process) is executed in an isolated sandbox environment, separated from other processes. Android OS assigns a different User Identifier (UID) ${ }^{8}$ and a directory to each application (except for applications coming from the same developer where a UID can be shared). At the same time, all its files are protected against unauthorized access from other applications in such a way that only the assigned UID can access them. More precisely, the UNIX-based permission model is enforced by the Kernel through the Uniform Resource Identifier (URI) [71] permissions that allow an application to application interaction, giving the ability to an application to grant selective access to data resources it owns.

In addition to the aforementioned Discretionary Access Control (DAC) mechanism, in the Android platform a Mandatory Access Control (MAC) has been implemented upon SELinux since Android 4.3. The MAC is enforced by system-level security policies that enforce the default deny [5]. Thus, SELinux provides a centralized policy configuration approach stronger than DAC for isolating and sandboxing Android applications and privileged Android system daemons. More precisely, it uses roles based on identities to limit their access to domains (processes) or types (for objects, i.e., , files, folders, etc.). Moreover, SELinux grants permissions based on UIDs or other security-relevant information [71] about each process where only explicitly defined actions can be performed.

Keymaster implements the Android key store into the Trusted Execution Environment (TEE). This mechanism stores cryptographic keys into TEE and protects them from unauthorized access, and even if a process is compromised (including Kernel), an attacker cannot read key material stored in Keymaster. Keystore [19] is the crypto-service API in the Android framework based on Keymaster Hardware Abstraction Layer (HAL). The security of Android Keystore is highly dependent on SELinux enforcement, and dedicated policies ${ }^{9}$ safeguard it. In the Android framework, a process can gain access to other processes Keystore if this is explicitly defined. This is a standard method whenever a privileged process is dependent upon or manages other processes. Such an example is the defined rules that allow system services to handle the keys of VPN (Virtual Private Network), Bluetooth, and WiFi services based on their UIDs. Another implementation of the Android key store is the Strongbox imported into Android version 9.0 that implements the Android Keymaster Hardware Abstraction Layer

\footnotetext{
${ }^{8}$ https://android.googlesource.com/platform/system/core/+/refs/tags/android-11.0.0_r3/libcutils/ include/private/android_filesystem_config.h

${ }^{9} \mathrm{https}$ ://android.googlesource.com/platform/system/security/+/refs/tags/android-11.0.0_r3/keystore/ permissions.cpp
} 
(HAL) into a dedicated tamper-resistant hardware security module (HSM) for supported devices.

The verified boot is the security mechanism that provides a chain of trust since the introduction of Android 4.4 by ensuring the firmware's integrity and authenticity from the bootloader to the boot, system, vendor and OEM partitions with the aid of TEE enforced at the kernel level [71]. More specifically, during the device boot, every stage verifies the upcoming stage's integrity before its execution.

The Gatekeeper [17] or Weaver implements the authentication of the end-user ownership of the device. The first case implements user lock screen verification based on PIN or password or pattern with Keymaster for TEE, while the latter with HSM upon Strongbox [71]. After the successful end-user authentication, Gatekeeper or Weaver communicates this to Keymaster or Strongbox to grant access to authentication-related keys.

Data encryption at rest was implemented initially in Android 4.4 with the Full Disk Encryption (FDE) mechanism that uses a protected credential key to encrypt the entire user data partition. The use of Trusted Execution Environment's (TEE) signing capability was firstly introduced in Android 5.0. This mechanism's main disadvantage was the core device services' inability to operate until the device's end-user provided a valid password entry [71]. The Full Disk Encryption mechanism was replaced by the File-Based Encryption (FBE) mechanism in Android 7.0 that leverages TEE or HSM to protect individual files with credentials by different users. File-Based Encryption provides cryptographical protection of per-user data on Android devices.

\subsubsection{Application Security}

Another class of permissions in the Android platform is the Application-Level Permissions or more specific Android permissions [20]. In this model, applications should "request" the suitable permissions to interact with sensitive system services, other applications, and components. This means that programmers should declare the necessary permissions (both normal and dangerous ones) in the application's manifest file if they target devices relying on Android Lollipop and previous versions in order to gain access to protected resources. From Android 6.0 onwards, programmers should define the permissions at the application's manifest file and in cases where "dangerous" permissions [20] are used to protect a resource these permissions should be requested at run time. The permissions requested by applications are granted by users either during the installation of the application or at runtime, depending on the Android version that they rely on. For instance, if an application reads the device ID (e.g., IMEI), it requires the READ_PHONE_STATE permission to be defined in its manifest 
file and the user to grant it at runtime otherwise, a security exception will be raised during execution.

Android application signing [21] is a mechanism enforced in the Android Framework to ensure that only applications from legitimate sources can be executed into a mobile device. Applications from non-trusted sources can be executed into Android devices only when the developer option is enabled for testing purposes. As part of the System Service, the Package Manager checks the application's signature's validity based on their carrying certificate during installation. Currently, Android does not provide Certificate Authority (CA) verification for application certificates, and all of them are self-signed and generated by OEMs, vendors, or developers. With this Public Key Infrastructure (PKI) oriented mechanism, Android OS can ensure each application's ownership onto a device. After the successful signature validation process, the system assigns UIDs into applications and enforces the isolation sandbox. An application that shares the same certificate with others can share the same UID if this is explicitly defined in its manifest file. In addition, applications that share the same UID can share resources such as preferences, TEE or Strongbox keys, etc.

\subsubsection{Exploit Prevention}

The most effective security mechanisms regarding the prevention of stack and heap-based vulnerabilities exploitation on Android are presented here, while more comprehensive lists can be found in $[27,6,8-15,7]$.

Stack Canary is a prevention mechanism that usually places random bytes (QWORD) based on /dev/urandom into a stack frame before the return address of an invoked function and checks if the contents are changed before the function returns. The android platform uses random canary since the introduction of version 4.0 [36].

Hardware-based No eXecute (NX) prevents code execution on the stack and heap, and it was introduced in Android version 2.3 [6]. With this mechanism, memory sections (pages) of the process which contain code are marked as executable and read-only [45]. In other words, a memory page can be marked as executable or writable.

Another prevention mechanism against code-reusing attacks that complement the NX mechanism was imported into the Android platform in version 4.0 called Address Space Layout Randomization (ASLR). This mechanism randomizes the locations of memory areas within an address space to make it probabilistically difficult for an attacker to gain control of a process [60]. 


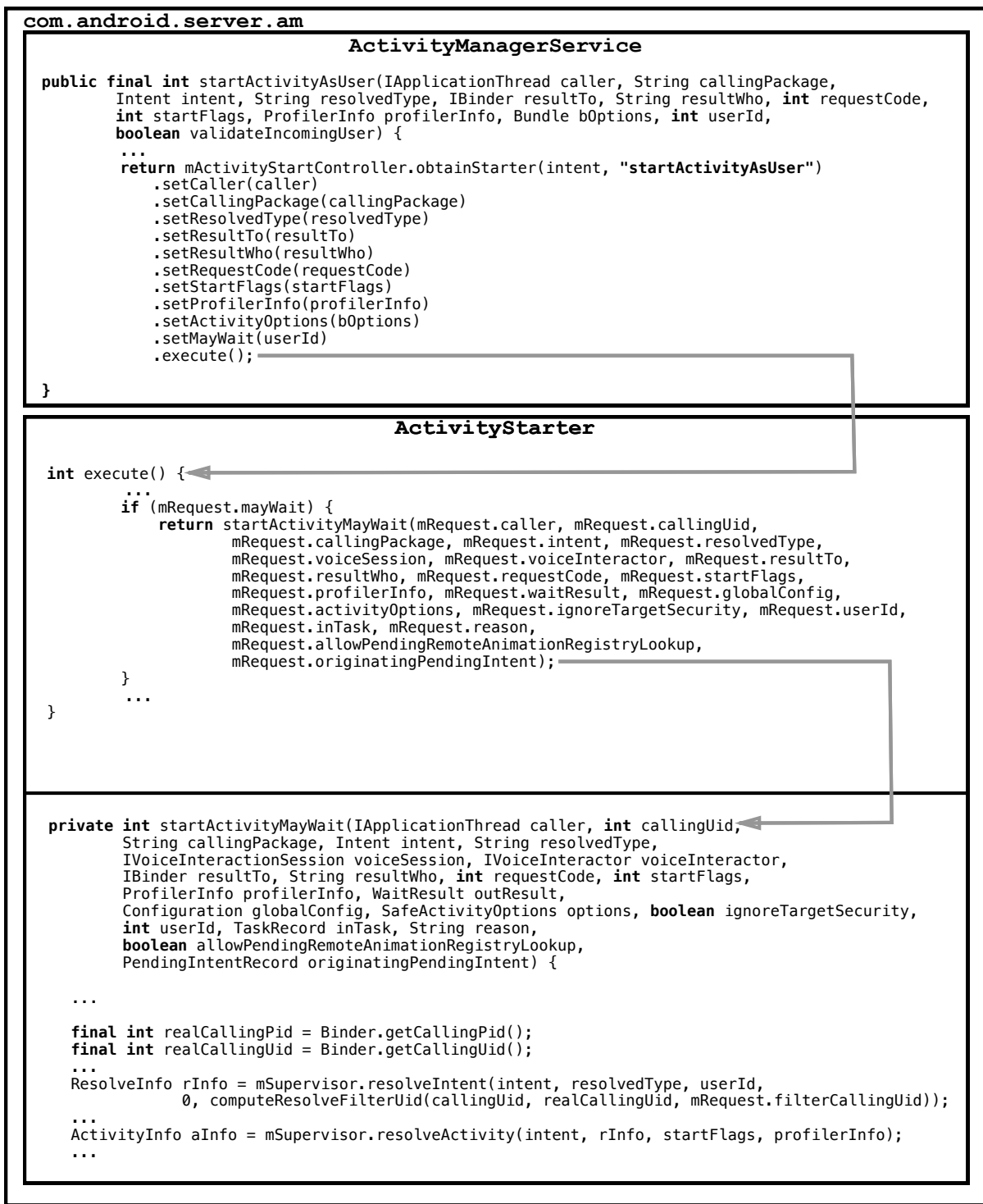

Figure 3.3 Android (Version 9) Activity Initialization and Task Handling. 



\section{Chapter 4}

\section{Android Permission Model}

Android Permission Model is a vital security mechanism for end user's privacy protection. Programmers get information on the correlation between permissions and protected Application Programming Interface (API) methods through Android's documentation. However a full correlation between the Android's Software Development Kit (SDK) API methods and permissions doesn't exist. This is caused by the fact that (a) documentation may accidentally lack information, and (b) Android has hidden and internal API methods that are not directly accessible at the application layer since they are not included in the Development SDK. Though the latter cannot be directly accessed there are several publicly available sources that give guidelines ${ }^{1}$ on how to gain access to such resources [58]. So eventually, programmers can gain access to these hidden API methods.

Moreover, as the Android OS evolves and in order to improve end users' experiences, it proceeds with various modifications to the underlying subsystems. For instance, permissions are enabled dynamically on the latest versions of Android, while from version 6.0 backwards they were granted statically. In addition, some API methods are deprecated, while other are introduced to support additional functionalities. So it is evident that these types of changes not only affect the API methods and permission mapping but also introduce inconsistencies in it among different Android versions according to [100].

\subsection{System Level Permission Enforcement}

Android utilizes the Application-Level Permission model to restrict access to device's sensitive features from untrusted applications. Specifically, Android as a service oriented framework enforces permissions' control at the lower stack layers; at Android framework level [82], without

\footnotetext{
${ }^{1}$ Android Hidden APIs: https://github.com/anggrayudi/android-hidden-api
} 
providing a centralized point for accomplishing this task. For instance, at the Android framework layer there are several API methods (enforceCallingOrSelfPermission(...), enforcePermission(...), enforceCallingPermission(...), etc.,) that impose permission controls declared in the Context ${ }^{2}$ Interface which is responsible to expose methods for accessing the underlying resources.

Figure 4.1 illustrates a high level example of a permission enforcement procedure. According to this example the application invokes the getIsimImpi ()$^{3}$ API method using Java reflection technique (this specific method cannot be found in the public documentation of the Android framework as it is not part of the public API) which is responsible to call the relevant resource implemented in the service side. In this specific case, the latter performs a permission control through the enforceCallingOrSelfPermission() API method. If the application has not defined the corresponding permissions (i.e., READ_PRIVILEGED_PHONE_STATE) the framework will cause a security exception reporting in the exception stack the missing permission. Note that not all the enforcement points raise such a security exception if a permission is missing; for instance this is the case of checkPermission() Framework method.

\subsection{Android Application Permission Acquisition}

Access to any sensitive resource of Android Framework is granted through a protected Application Programming Interface (API) method. An application in order to use a protected API method it must first declare the corresponding permissions in its manifest file, and request it also at runtime if it is executed on Android latest versions, otherwise a security exception is raised. In any case, users should give their consent for the permissions requested by the application either during the first time that a protected API method is invoked or during installation process, depending on the Android version [38, 100].

\subsection{Threats of Android Permission Model}

\subsubsection{Permission Re-Delegation}

The threat of permission re-delegation is a form of confused deputy attack [47, 91] or similarly privilege escalation attack. In the Android platform, the threat of permission re-delegation

\footnotetext{
${ }^{2}$ https://android.googlesource.com/platform/frameworks/base.git/+/refs/tags/android-7.0.0_r34/core/ java/android/content/Context. java

${ }_{3}$ https://android.googlesource.com/platform/frameworks/base.git/+/refs/tags/android-7.0.0_r34/ telephony/java/android/telephony/TelephonyManager. java
} 


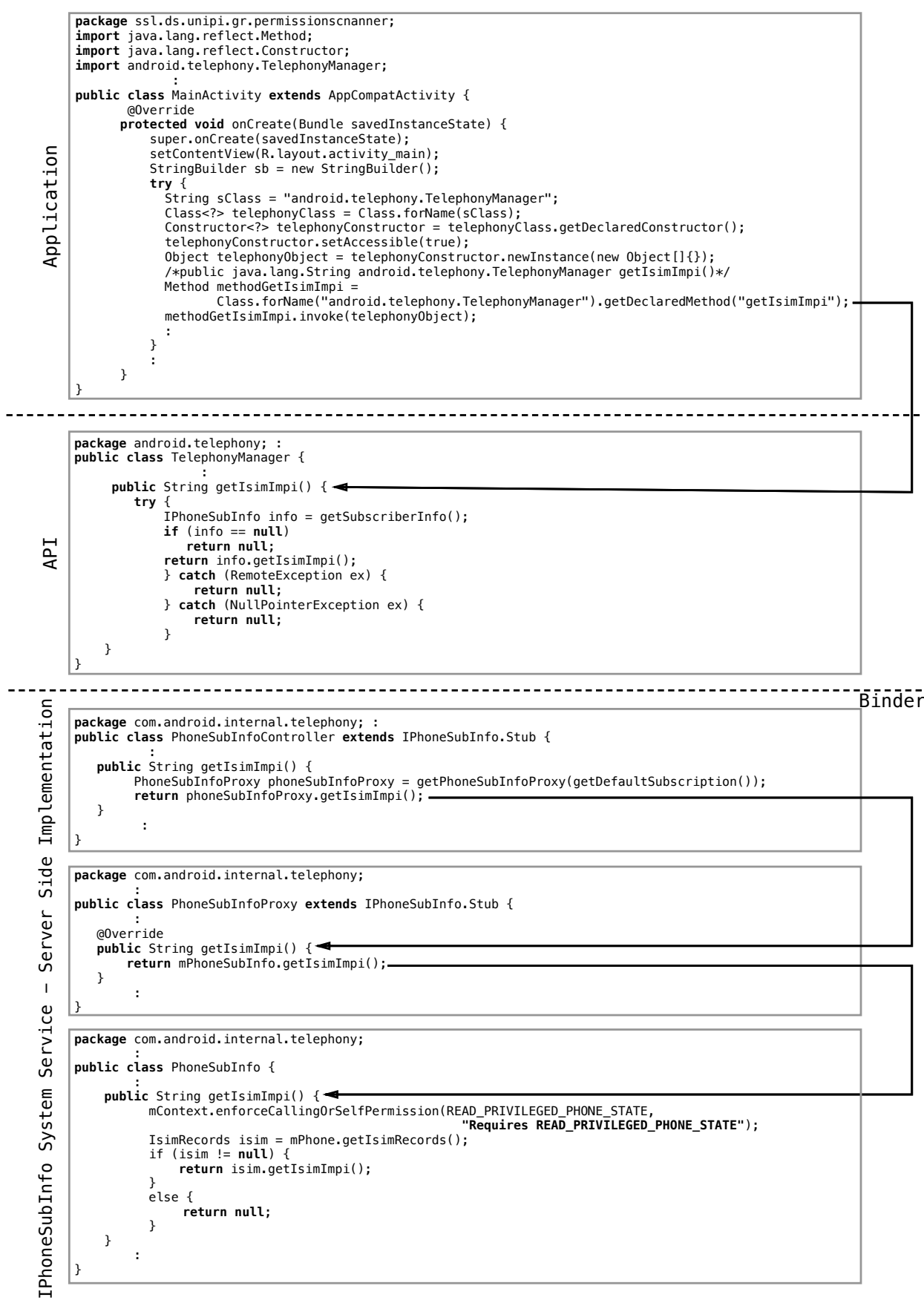

Figure 4.1 Android (Version 7) Permission Enforcement Control Point Example.

occurs whenever an application with granted permission performs an action on behalf of a less privileged application [42]. This kind of threat can be materialised in several ways: (a) An application may intentionally expose sensitive functionality, and a malicious application leverages it in order to perform a non-legitimate action. (b) A developer may accidentally 
expose a sensitive functionality that can be exploited by an intrusive application. In any of the cases mentioned above, permission re-delegation is a threat with a severe impact on end users' privacy because the Android framework does not restrict application with fewer permissions to access more privileged application's components [32]. This type of attacks is primarily linked with design errors [49] by the application developers that ignore security recommendations and best practices suggestions.

\subsubsection{Over-Privileged Android Applications}

An application is considered over-privileged whenever it declares more than the needed permissions for its normal functionality $[41,44]$. This could increase vulnerabilities as well as security and privacy risks in those applications [90]. The main causes of over-privileged applications are development misconfigurations, poor documentation regarding the correlation between Android permissions and API methods, or lack of knowledge on application developers' behalf about application security development best practices. Several research works have tried to detect over-privileged applications with static [24, 26], dynamic [41], or hybrid analysis [44].

\subsection{Android Permission Model Mapping}

The correlation between Android permissions and API methods is defined as Permission Map and is undoubtedly a vital component for Android's application security assessment e.g., overprivilleges [24, 26, 94, 41, 44], malware detection [23, 77, 57], etc., since it can identify inconsistencies, misconfigurations and other flaws. Thus, several researchers embrace either a static or a dynamic approach in order to correlate Android APIs with the corresponding permissions, and construct the so-called permission map.

Solutions adopting the static approach perform a full analysis [24, 26, 25] of the Android OS source code. Though such solutions can achieve high coverage, they don't have a way to validate their outcomes; in other words, they suffer of false positives pairs. On top of that, in many cases $[24,26]$ they require the identification of Android's permission enforcement functions beforehand, otherwise their analysis fails. Or other's scope of analysis is quite limited despite their accuracy [29]. Approaches adopting the dynamic analysis [41, 65] are free of false positives but, depending on their characteristics, may not achieve to cover the full set of protected API methods. The latter it is also limitation of [65] which in fact cannot successfully generate permission mapping for APIs methods requiring specific conditions to be met i.e., in case where an API method requires a previous event to be triggered for its 
execution afterwards. The comparison among the proposed methods regarding the Android permission mapping generation is listed in Table 4.1 .

\subsubsection{Dynamic Analysis Based Approaches}

Stowaway [41] is the very first work that develops a map between protected APIs and permissions in the context of detecting over-privileged applications. Stowaway builds a solution considering the advantages of Java tests and Randoop [76] in order to compile the appropriate tests and dynamically invoke the APIs incorporated in a given application. However, Stowaway needs modifications to the underlying OS in order to monitor the permission enforcement mechanism and thus identify which permission is required for a specific Android's API. This method achieves $85 \%$ coverage of the Android API, but it also requires manual testing for the permission map identification and manual effort to be adaptable to a variety of Android Open Source Project (AOSP) versions. It is important to stress that Stowaway is no longer supported and thus the provided permission map is totally outdated ${ }^{4}$.

Lyvas et al. [65] introduced an open-source orthogonal to the existing approaches framework, named Dypermin, which builds on the advantages of the Android framework features to generate the permission map. Dypermin relies on: (a) the security exceptions raised by the Android OS during the execution of an application that lacks the necessary permissions for invoking protected APIs, (b) the capability to access any protected API i.e., publicly accessible or hidden, through the Android framework, and (c) the Java reflection technique for invoking protected APIs at run time.

Using the aforementioned features, Dypermin generates and invokes the appropriate objects and APIs embedded in an application, and inspects whether a security exception is raised. In this way, no modifications to the underlying Android OS is required and thus the proposed methodology can be applied to any unmodified Android device, emulator or Virtual Machine without needing root access.

As depicted in Figure 4.2 Dypermin's operation can be abstracted in three phases. Specifically, during the first phase (steps 1-2) Dypermin extracts from the Android framework all the packages and their classes with their corresponding APIs, including additional related information such as arguments, modifiers, class members, returning values as well as annotations and comments, and stores them in a collection named SDK dump. Moreover, during this phase Dypermin processes also API's documentation; that is the Javadoc comments of API's methods, and identifies if a given method is publicly accessible or hidden (based on "@hide" document annotations). Additionally, it extracts all the permissions per method that

\footnotetext{
${ }^{4}$ http://www . android-permissions.org
} 
are defined in comments or method annotations. In this way, Dypermin is able to compile the complete list of available methods.

To generate a signature for any available method of the Android framework, Dypermin utilizes a custom made tool based on the javalang python parser package.

In this point it is crucial to recall that the Android framework contains both publicly accessible and hidden protected API methods, and also that the hidden API methods are excluded from the Android development SDK library (android.jar), meaning that they cannot be directly invoked by an application. To bypass this restriction developers have two options:

1. either use the Java reflection mechanism that enables an application to load, at runtime, the corresponding API from the devices framework. jar classes, or

2. replace the original Android development SDK library (android.jar) with a custom one that includes all Android APIs.

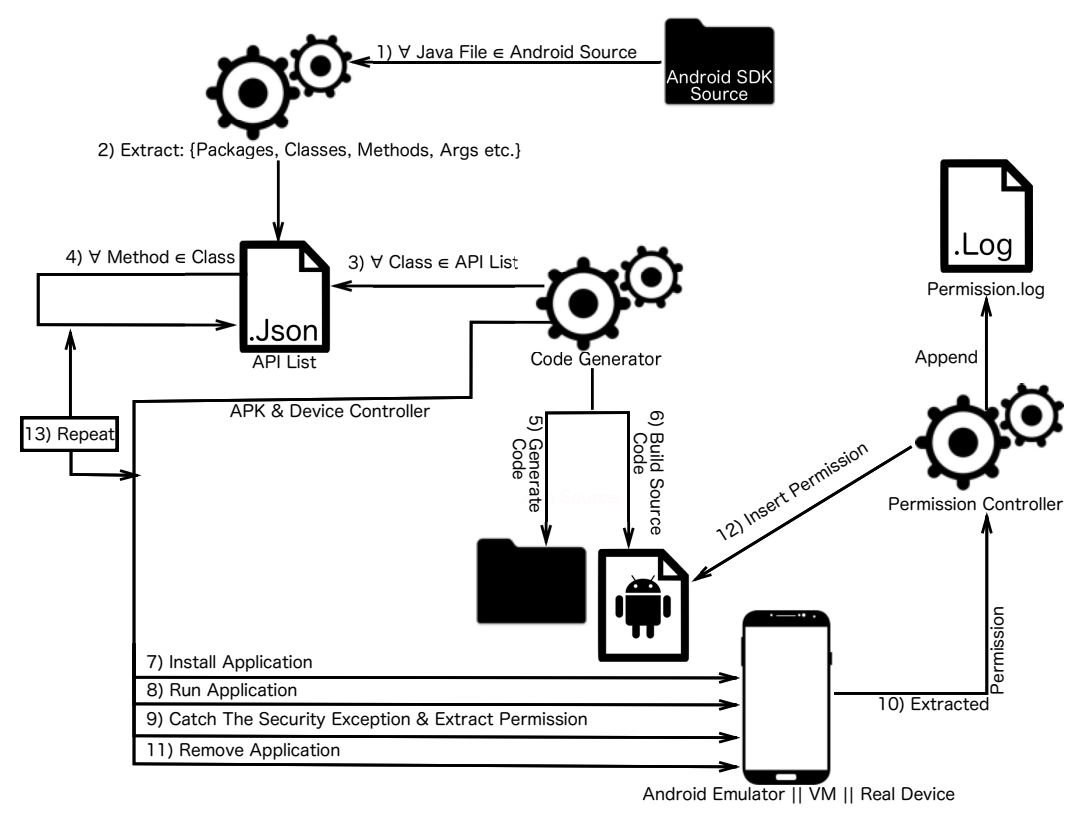

Figure 4.2 Dypermin's Design for Permission Map Compilation

The proposed framework, has adopted the Java reflection approach since it is transparent to the underlying OS and it does not pose a need for modifying the device's OS.

During the second phase (steps 3-5), Dypermin identifies all the available constructors for the corresponding APIs, using the SDK dump collection that was generated during the first phase. Dypermin in order to instantiate an object of a given class first prioritize the 
candidate constructors based on the type of arguments that they contain in the following way: (a) Firstly, constructors with arguments that belong to Java basic datatypes (int, long, char etc.) or belong to android. content. Context and/or java. lang. String class. (b) Secondly, constructors with basic datatypes or arguments from Context and/or from classes that belongs to java.lang package. (c) Thirdly comes constructors that they dont' require arguments. (d) Finally, any constructor that do not contain arguments from abstract classes or belong to interfaces.

Dypermin in order to instantiate the objects of a given constructor, it first generates the object instantiation code of the involved arguments in a recursive way, as Listing 4.1 depicts.

If more than one available constructor belongs in the same category Dypermin prioritize them in those that belongs to the publicly accessible API and then to those from the hidden API. Using such an approach it is possible to generate automatically valid code for almost any object. Furthermore it should be stressed that in cases where constructors or methods of the corresponding classes require access to a valid Context class object, an instance of an application context is passed. Otherwise, object instantiation either fails or it does not trigger a security exception, since the context object is null. For method invocation where objects are required as parameters, Dypermin generates them in the same way as above.

In cases where classes have constructors with abstract class or interface arguments the aforementioned heuristic cannot be applied for their instantiation. This is leveraged by the fact that in most cases the required constant values associated with the manager class are hardcoded in the class code. Dypermin is able to parse the under-analysis class code and extract those tokens and use them to create a valid object of the given class.

During the third phase, Dypermin employs the toolkit Gradle [18] for building applications (step 6), that call (through the source code generated in the second phase) the methods that are necessary i.e., ( onCreate () method - see Figure 4.1) for the invocation of the examined APIs. These applications do not specify any permissions in their manifest files and handle security exceptions in a try ... catch statement. For every security exception all relative data are kept in a log file. The applications are installed and executed (steps 7-8), through the Android Debug Bridge (adb), the APIs under examination are invoked, and the status is monitored through the analysis of the log files. Utilizing the fact that the Android framework raises a security exception if a protected API is invoked without the appropriate permissions appearing in the application's manifest file, Dypermin identifies the permission that caused the security exception and correlates it with the examined API (step 10).

In step 9-10, Dypermin defines the identified missing permissions in the application's manifest file and repeats the execution of the applications until no more security exceptions appear. In this way Dypermin can also identify protected APIs that are correlated with 
multiple permissions. Dypermin revokes the application (step 11) from the device after the first successful invocation of the examined API.

The above-mentioned method is also suitable for mining multiple permission for a given API method. The high-level overview of this functionality is as follows. For every method invocation that triggers a Security Exception the Dypermin Proof-of-concept implementation would be able to import it in the application's manifest file and repeat this process until no new security exceptions occur. This practice is able to discover all needed permissions (if multiple permissions required) for any method (as long as a security exception occurs).

An example of Dypermin's auto generated application code is depicted in Listing 4.1. In particular, in line 7 the method getSystemService (. . ) was used to retrieve of the systemlevel service by its name, with a new instance of the class ConnectivityManager ${ }^{5}$, able to handle the management of network connections. From line 11 to 75 Dypermin generates in a recursive way, the object instantiation code of the involved arguments of the method registerNetworkAgent (..). The method to be invoked is declared by its name and its parameter's signature (lines 80-86). Then all the generated class objects (lines 11-75) are used to call the method registerNetworkAgent ( . . ) (lines 87-92). If any security exceptions are raised they will be handled at the catch block (lines 100 to 135), identifying the permissions that are missing from the application's manifest file.

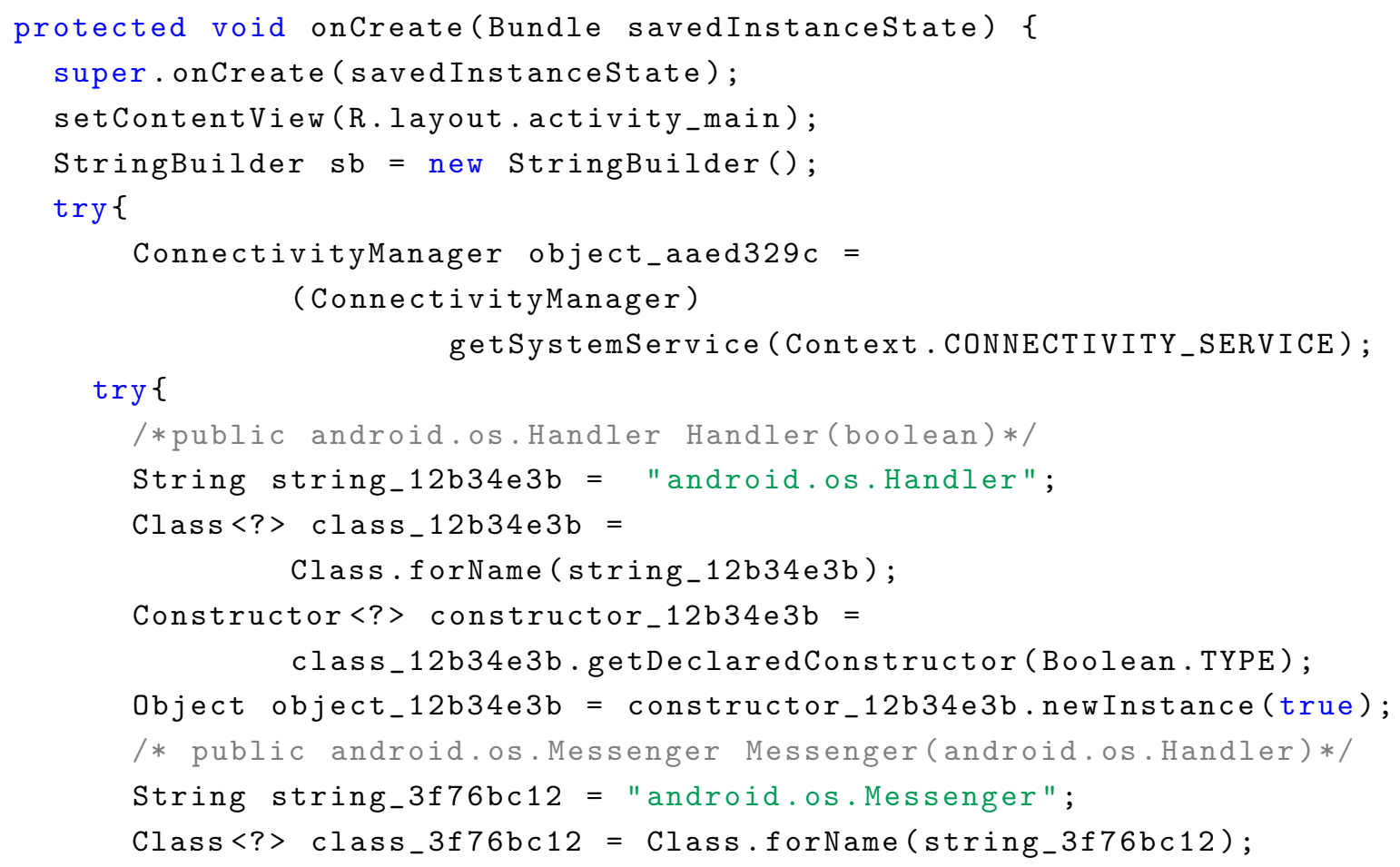

$5_{\text {https://android.googlesource.com/platform/frameworks/base.git/+/refs/tags/android-7.0.0_r34/core/ }}$ java/android/net/ConnectivityManager.java 
Constructor $<$ ? > constructor_3f $76 \mathrm{bc12}=$ class_3f $76 \mathrm{bc} 12$.getDeclaredConstructor (Handler.class); 


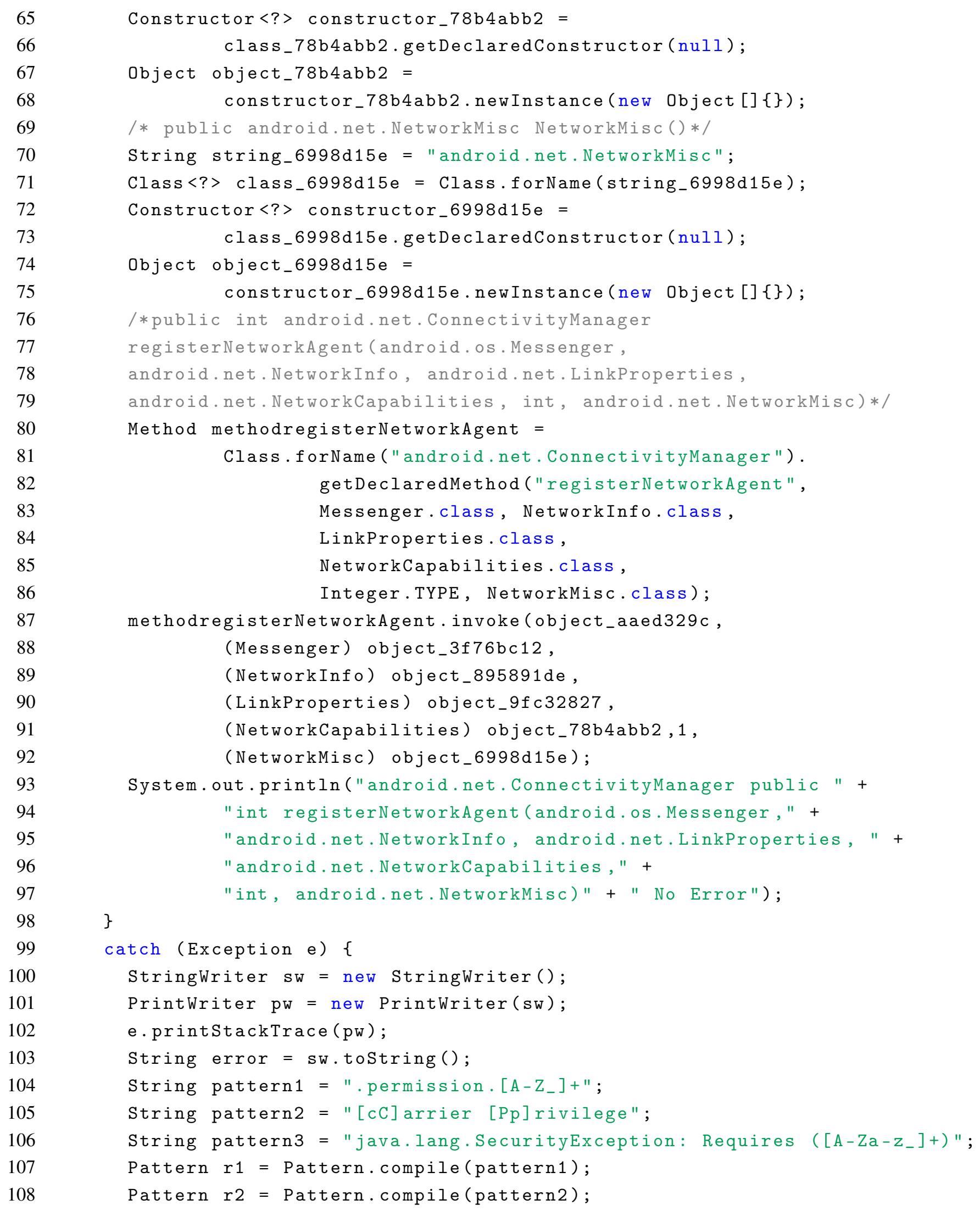




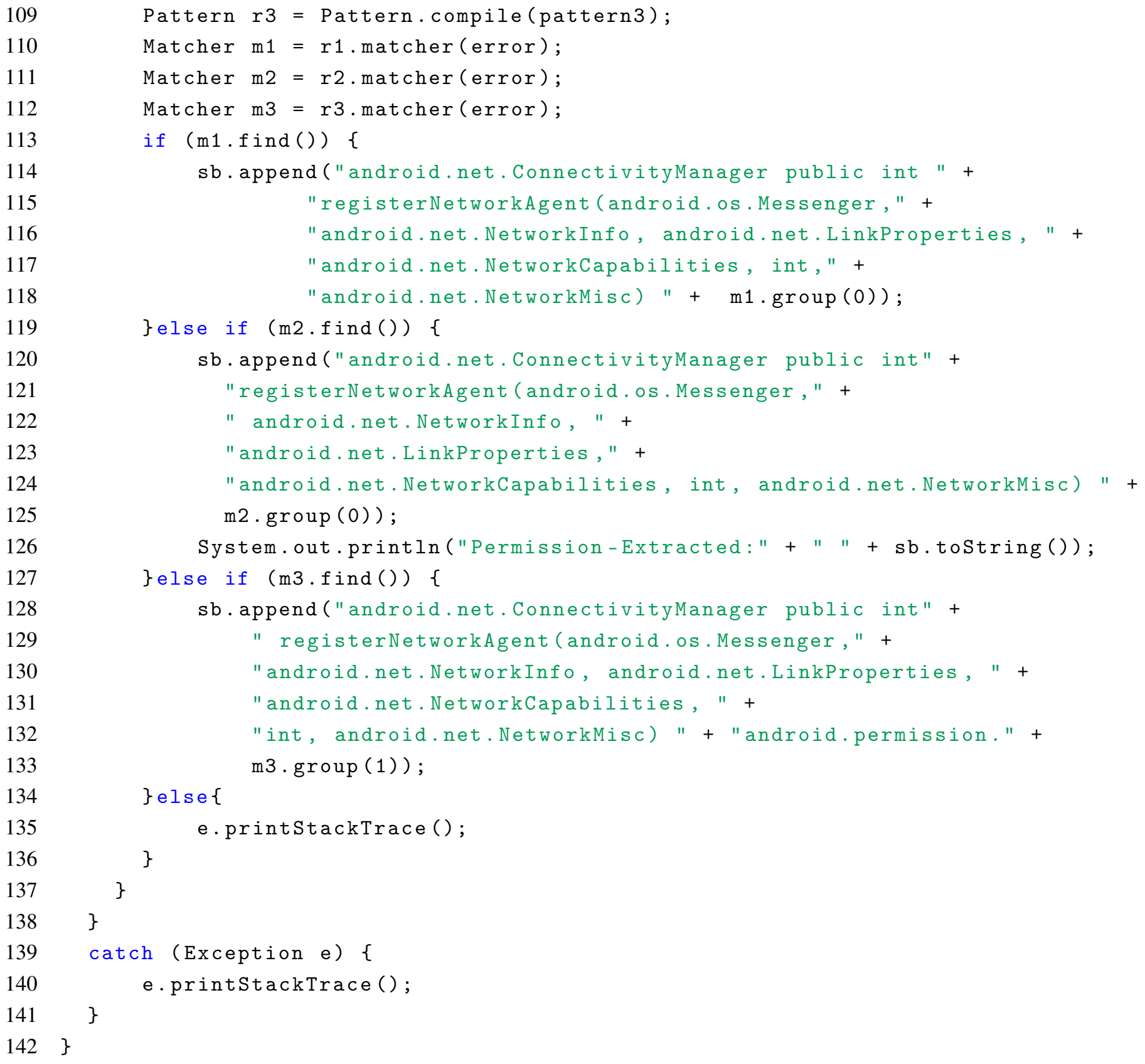

Listing 4.1 Code Generated by Dypermin For registerNetworkAgent ( . . ) Private API Method.

\subsubsection{Static Analysis Based Approaches}

While only Stowaway [41] and Dypermin [65] utilize dynamic analysis, there are few other methods such as PScout [24], Bartel et al.[26], and Axplorer [25] that use static analysis of Android OS source code to derive the permission map. 
PScout [24] is the first tool that could perform Java bytecode analysis with the aid of Soot framework [87] and generate a control flow graph (CFG) based on Class Hierarchy Analysis (CHA) [33] of the Android framework, including Binder Inter Process Communication (IPC) and Remote Procedure Calls (RPCs). For its initialization, PScout requires the identification of all possible permission enforcement points of the Android framework in order to approximate whether or not a particular API invocation triggers a permission check using a backward reachability traversal approach over the CFG. Though someone may consider PScout's permission map partially outdated, since the latest list available online is for Android version 4.1.1 ${ }^{6}$, it is worth noting that in several cases it is an essential part of other research works that focus on privacy leaks identification, and malware detection [59, 99]. PScout is based on several assumptions for mapping between protected API methods and permissions that may produces false positives.

Similarly, Bartel et al. [26] utilize different static analysis methods to extract the permission map. Specifically, they have generated a CFG based on CHA for Android version 4.0.1 at bytecode level, in several variations (CHA-Naive, CHA-Android and Spark-Android) with the aid of Soot [87] and Spark [56]. Firstly, they performed a string analysis to mine the permission names from the CFG. Then, they carried out a service redirection to pair the service caller to services. Finally, with service identity inversion they removed the unnecessary code from their initial CFG. Although in their research they provide all the necessary evidence that their analysis produces reliable results, they have neither published the code of their method, nor they have provided any experimental verification. Finally, the generated permission map is not any longer available ${ }^{7}$. Additionally, the proposed framework is unable to detect dynamic code loading.

Axplorer [25] relies on the static analysis of the Android framework, as the aforementioned methods do, aiming, however, at a much higher precision. In contrast to other solutions, Axplorer conducts an in-depth static analysis of the Android framework by constructing inter procedural CFGs. In order to improve precision, instead of analyzing the CHA, Axplorer exploits the advantages of object sensitive pointer resolution [73]. It is important to stress that only the public API methods are included in the analysis while hidden API methods are excluded. Furthermore, Axplorer results highlight that the PScout's [24] permission map contains inconsistencies; mainly false positive identifications.

Last but by no means least, another work that focus on Android permission mapping is presented in [29]. A solution (named DPSpec) in which the permission mapping is generated based on XML annotations and Javadoc comments of Android SDK is introduced. Although,

\footnotetext{
$6_{\text {http: } / / \text { pscout.csl.toronto.edu }}$

${ }^{7}$ https://www . abartel.net/permissionmap/
} 
authors claim higher accuracy compared to Axplorer they do not provide neither the source code of their tool nor the permission map. The proposed tool is limited to construct permission maps from Android's SDK source code annotations, Javadoc comments and XML annotations only.

\begin{tabular}{|c|c|c|c|c|}
\hline Solution & Characteristics & Android Version & Availability & Adaptability \\
\hline Stowaway [41] & $\begin{array}{l}\text { Dynamic analysis framework based } \\
\text { on reflection (method invocation) } \\
\text { and modifications of the underlying } \\
\text { OS (permission enforcement points } \\
\text { monitoring). It requires manual ef- } \\
\text { fort to be adaptable to a variety of } \\
\text { AOSP versions. }\end{array}$ & 2.2 & $\begin{array}{l}\text { Not available source code nor } \\
\text { permission maps. }\end{array}$ & No \\
\hline PScout [24] & $\begin{array}{l}\text { Static analysis framework based on } \\
\text { CFGs and CHA with several as- } \\
\text { sumptions for mapping between pro- } \\
\text { tected API methods and permis- } \\
\text { sions. }\end{array}$ & $2.2 .3-5.1 .1$ & $\begin{array}{l}\text { Available source code and per- } \\
\text { mission maps. }\end{array}$ & Unknown \\
\hline Bartel et al. [26] & $\begin{array}{l}\text { Static analysis framework based on } \\
\text { CFGs and CHA in several variations } \\
\text { unable to detect dynamic code load- } \\
\text { ing. }\end{array}$ & 4.0 .1 & $\begin{array}{l}\text { Not available source code nor } \\
\text { permission maps. }\end{array}$ & Unknown \\
\hline Axplorer [25] & $\begin{array}{l}\text { Static analysis framework based on } \\
\text { inter procedural CFGs and sensitive } \\
\text { pointer resolution designed to anal- } \\
\text { yse only the public API methods. }\end{array}$ & $4.1 .1-6.0$ & $\begin{array}{l}\text { Not available source code but } \\
\text { available permission maps. }\end{array}$ & Unknown \\
\hline DPSpec [29] & $\begin{array}{l}\text { Static analysis tool able to parse and } \\
\text { construct permission maps from An- } \\
\text { droid's SDK source code annota- } \\
\text { tions, Javadoc comments and XML } \\
\text { annotations. }\end{array}$ & Any & $\begin{array}{l}\text { Not available source code nor } \\
\text { permission maps. }\end{array}$ & Yes \\
\hline Dypermin [65] & $\begin{array}{l}\text { Dynamic analysis framework based } \\
\text { on Android SDK source object cor- } \\
\text { relation map, reflection (method in- } \\
\text { vocation) and permission based Se- } \\
\text { curity Exception extraction. De- } \\
\text { pended on valid object generation. }\end{array}$ & Any & $\begin{array}{l}\text { Available source code but not } \\
\text { available permission maps. }\end{array}$ & Yes \\
\hline
\end{tabular}

Table 4.1 Method Comparison for Android Permission Mapping Generation

\subsubsection{Experimental Evaluation}

To assess Dypermin's accuracy, correctness and performance, it has been deployed on two different Android emulators configured with versions 4.1.1 and 6.0 correspondingly, run on an Intel Core i5 1,3 GHz CPU with 8 GB RAM. The selection of the above Android 
versions was based on the fact that they are considered as the major Android milestones, but also because other related methods, such as Stowaway [41], PScout [24], Axplorer [25] and Bartel et al. [26], were tested on the same versions. Furthermore, for the evaluation four managers i.e., TelephonyManager, WiFiManager, ConnectivityManager, and SmsManager and two other general purpose classes i.e., SystemVibrator and SipAudio, have been selected in order to demonstrate the completeness of the approach.

Through the Dypermin's code generation module, described in Subsection 4.4.1, and without loss of generality, the total number of classes, from the Android source code related packages $^{8}$, that the Dypermin tool could instantiate were counted with static analysis (based on the recursive calculation of class constructors and methods arguments). Specifically, for Android version 6.0, Dypermin would be able to generate code for 28170 classes. The total number of methods from classes that Dypermin was able to successfully calculate their instantiation in the previews step (static analysis and recursive computation based on their arguments also available in the SDK dump) were 247897 out of 276067 methods. The reason that Dypermin could not invoke $10.3 \%$ of the methods, is that they belong in abstract classes or that they contain arguments that belong to abstract classes or interfaces or their constructors cannot be invoked as well. The efficiency of Dypermin has been based on the static analysis since the compilation time of any generated application is relatively high, a fact already depicted in Figure 4.4 and Figure 4.5.

Since today a complete list of permissions related to protected API methods is not available, the evaluation of the accuracy of the proposed solution, as well as its comparison to other methods, has been solely based on the heuristic formula (1) that provides the full coverage of Android's Framework permissions. That is the set of protected API methods that according to Dypermin, other methods, the SDK source and other public documents, are requiring at least one permission.

\section{$\mid$ Dypermin $\cup$ SDKSource $\cup$ PublicDocumentation $\cup$ Other $\mid(1)$}

Note that none of the existing wokrs tries to accomplish such an holistic comparison. Results highlight that Dypermin can infer with high accuracy the map between protected API methods and permissions, as it overwhelms both public and SDK documentation (Figure 4.3). Specifically, according to formula (1) for the Android version 4.1.1 the identification rate for permissions - protected API methods, reaches 91.6\%, for the TelephonyManager class, 94.5\% for the WiFiManager class, $94.2 \%$ for the ConectivityManager class, $72.7 \%$ for the SmsManager class, $100 \%$ for the SystemVibrator Class and $12.5 \%$ for the SipAudioCal class.

\footnotetext{
${ }^{8}$ com. android.* , android.*
} 


\begin{tabular}{|l|c|c|c|}
\hline \multirow{2}{*}{\multicolumn{1}{|c|}{ Class Name }} & \multicolumn{3}{|c|}{ Android 6.0 } \\
\cline { 2 - 4 } & Dypermin & SDK Source & Documentation \\
\hline TelephonyManager & $91.6 \%$ & $66.6 \%$ & $37.5 \%$ \\
\hline WiFiManager & $94.5 \%$ & $0 \%$ & $0.02 \%$ \\
\hline ConectivityManager & $94.2 \%$ & $14.2 \%$ & $22.8 \%$ \\
\hline SmsManager & $72.7 \%$ & $27.2 \%$ & $27.2 \%$ \\
\hline SystemVibrator & $100 \%$ & $0 \%$ & $0 \%$ \\
\hline SipAudioCall & $12.5 \%$ & $12.5 \%$ & $12.5 \%$ \\
\hline
\end{tabular}

Table 4.2 Protected API methods coverage comparison between Dypermin and Android's available sources for Android version 4.1.1.

The corresponding results for the Android version 6.0 are 92.7\%, 100\%, 79.2\%, 57.1\% $100 \%$ and $33.3 \%$ respectively. It can be clearly noticed from Table 4.2 and Table 4.3 that in all cases public and SDK source documentation report a smaller number of protected API methods, as compared to Dypermin. In other words the currently available documentation is missing important information about the permissions that protected API methods are requiring for their execution, something that, as already explained, Dypermin can accurately identify eliminating any false positive results.

Moreover, Dypermin in this point it should be mentioned that is able to mine also system permissions, which are those used only by system applications. For instance, consider the case of a non-public API of the TelephonyManager class such as getCompleteVoiceMailNumber (int) for which Dypermin identifies as a required permission for its execution the "CALL_PRIVILEGED". Note that this permission is only available to system application, according to Android public documentation ${ }^{9}$ and as a result there is not any other way to retrieve this pair of API method - permission beforehand.

Regarding Dypermin's performance evaluation in terms of the time consumed for the generation of the permission map (object generation, application building, installation which is incorporated in the building time and execution), Figures 4.4 and 4.5 illustrate a snapshot

\footnotetext{
${ }^{9}$ https://developer.android.com/reference/android/Manifest.permission.html\#CALL_PRIVILEGE
} 


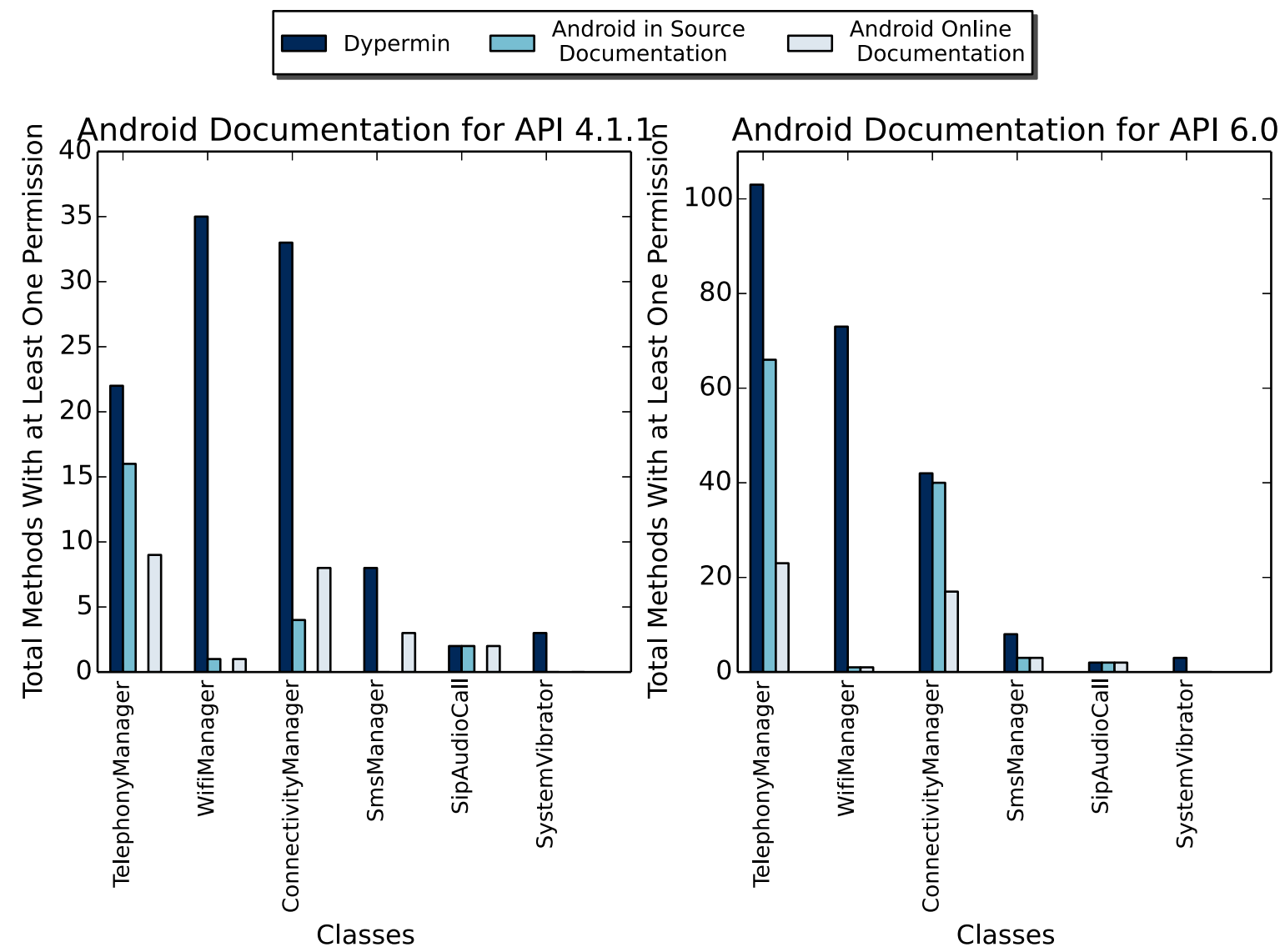

Figure 4.3 Total number of methods with at least one permission as identified by Dypermin in comparison with Android software development kit source code and its online counterpart documentation.

of the required processing time for each phase per different method ${ }^{10}$. Results are provided for the different Android versions and for all the classes that were tested. Briefly, outcomes demonstrate that Dypermin requires on average less than 17 seconds for code generation, building and testing for each method on both Android 4.1.1 and 6.0. For instance, the worst case scenario was for TelephonyManager class in which the code generation, building and testing consume up to $0.06,13.4$, and 3.8 seconds respectively. For the remaining classes that were tested the average processing time is slightly lower. Without loss of generality it can be noticed that the most time consuming phase is that of application's built, while the execution time varies depending on the method. Nevertheless the latter processed time can be considered negligible as it reaches up to $0.06 \mathrm{sec}$.

\footnotetext{
${ }^{10}$ Recall that for every method a new application is generated, installed and executed on an emulator.
} 


\begin{tabular}{|l|c|c|c|}
\hline \multirow{2}{*}{\multicolumn{1}{|c|}{ Class Name }} & \multicolumn{3}{|c|}{ Android 6.0 } \\
\cline { 2 - 4 } & Dypermin & SDK Source & Documentation \\
\hline TelephonyManager & $92.7 \%$ & $61.1 \%$ & $21.2 \%$ \\
\hline WiFiManager & $100 \%$ & $0.01 \%$ & $0.01 \%$ \\
\hline ConectivityManager & $79.2 \%$ & $75.4 \%$ & $32 \%$ \\
\hline SmsManager & $57.1 \%$ & $21.4 \%$ & $21.4 \%$ \\
\hline SystemVibrator & $100 \%$ & $0 \%$ & $0 \%$ \\
\hline SipAudioCall & $33.3 \%$ & $33.3 \%$ & $33.3 \%$ \\
\hline
\end{tabular}

Table 4.3 Protected API methods coverage comparison between Dypermin and Android's available sources for Android version 6.0.

Overall, Dypermin needs less than 17 seconds for generating the permission map for a single method. Eventhough such an overhead per method it can be considered insignificant, the generation of the permission map for the entire Android framework will require a very long processing. To eliminate this significant processing overhead the testing procedure can be parallelized, on different emulators. 

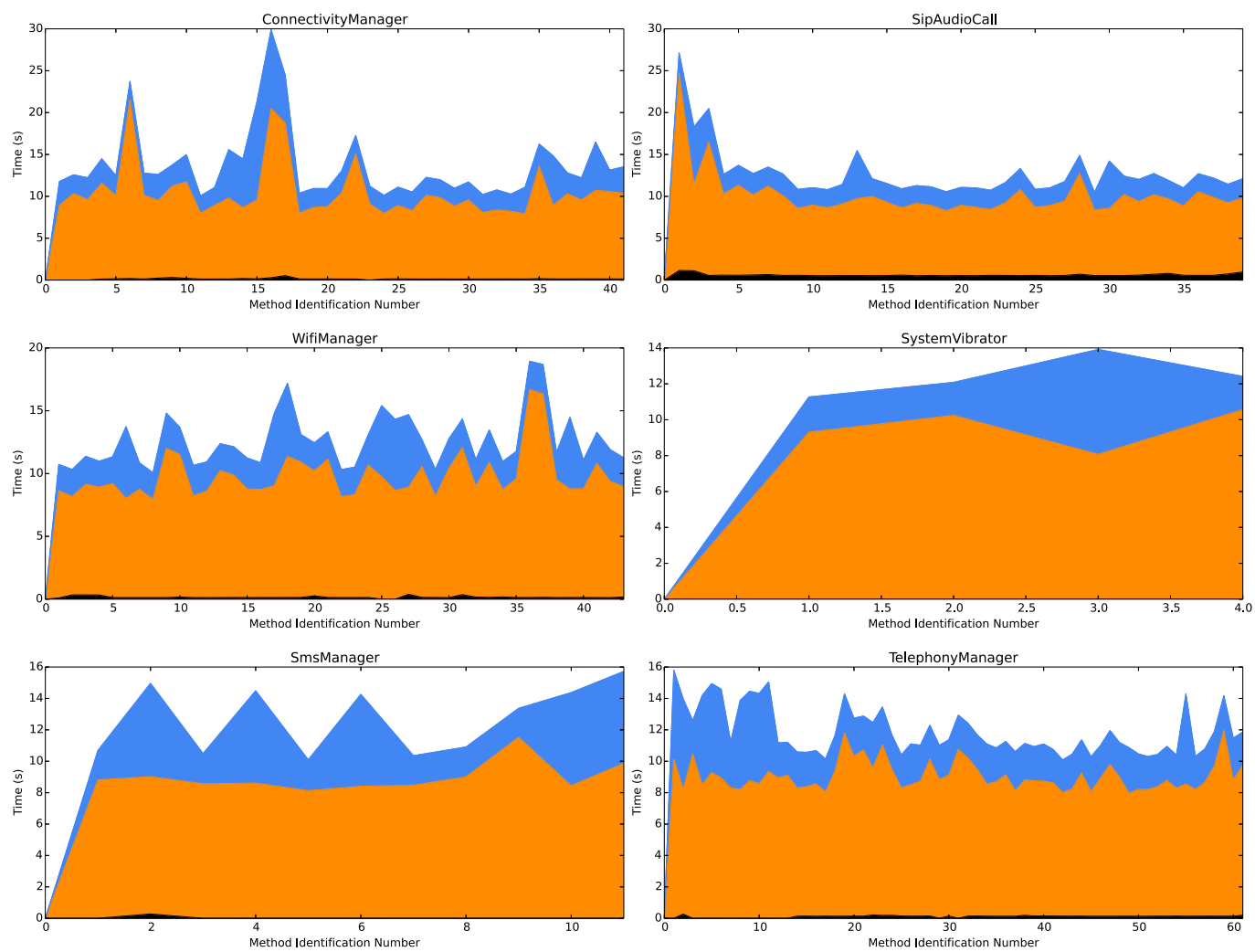

Figure 4.4 A snapshot of Dypermin's time performance per method on Android Version 4.1.1
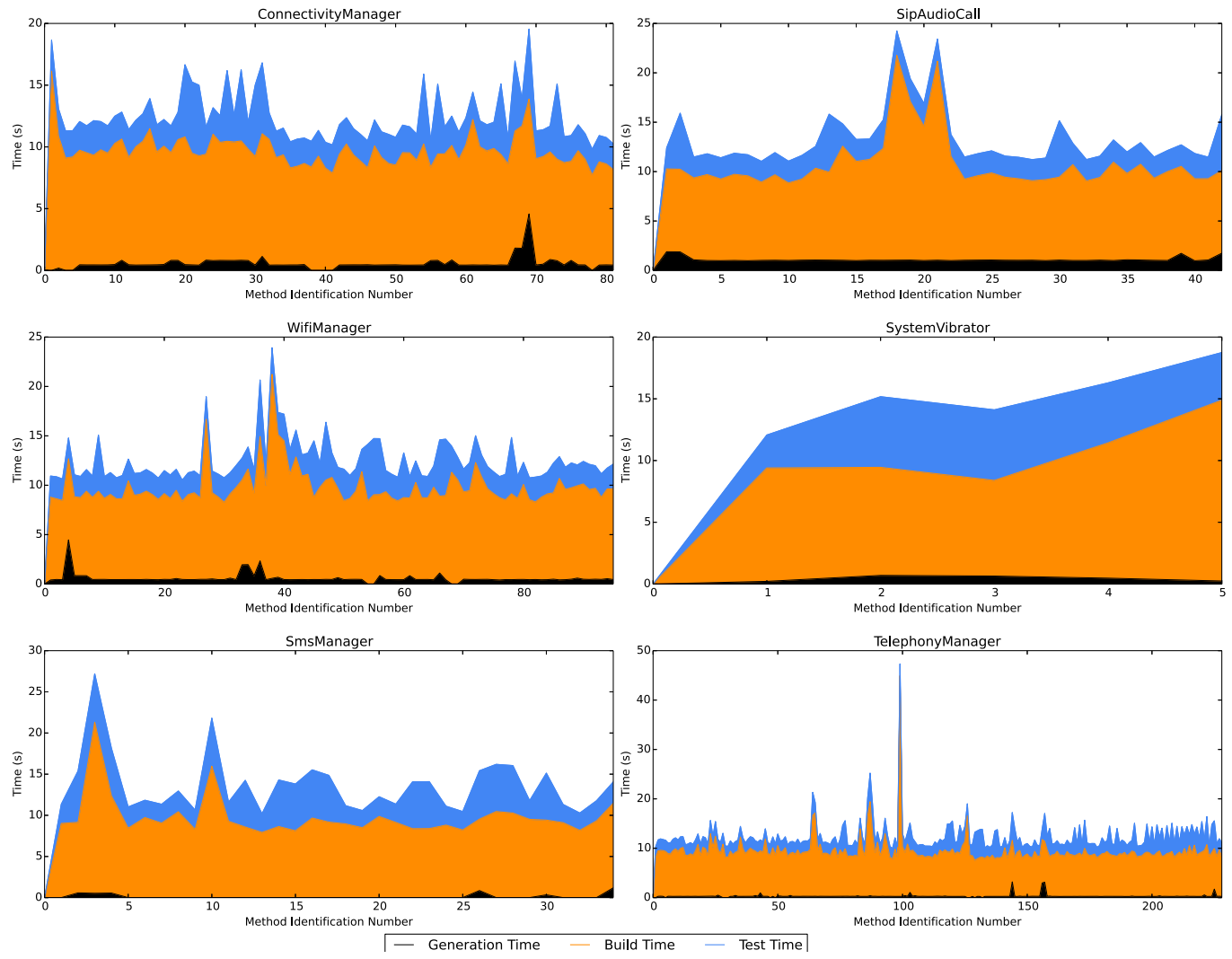

Figure 4.5 A snapshot of Dypermin's time performance per method on Android Version 6.0 


\section{Chapter 5}

\section{Android Task Management}

The Android framework offers the option to activities from different applications to coexist under the same task. This, unlike other OSes, refers to a multitasking mechanism for providing certain functionality i.e., to maintain the state of the activity without launching a new instance or clearing anything on top of it. Though such a mechanism, as already mentioned, offers several advantages i.e., seamless transfer between activities and applications, a malicious entity may try to manipulate it in order to launch hijacking attacks [81, 89, 79, 80, 55, 93, 61, 92]. The comparison of the methods, proposed in the literature, for detecting and preventing the Android task and activity hijacking attacks, can be found in Table 5.1.

\subsection{Threats of Android Task Management}

The research by Ren et al. [79] was the first that systematically study and address the activity hijacking vulnerabilities in Android OS. Attacks against Android's task handling mechanism can be accomplished by manipulating the attributes of $\langle\text { activity }\rangle^{1}$ elements, like taskAffinity, launchMode, etc., defined in the target application's manifest and/or intent flags. Activity's attributes, among others, define in which task the activity belongs. By default all app's activities belong to the same task. Whenever the taskAffinity attribute points to a different package name from the one to which it belongs, the initiated activity is assigned to a different task affinity. Note that in cases where the taskAffinity attribute is combined with the allowTaskReparenting, the activity is pushed to the targeted application's task stack; that is defined in the taskAffinity attribute.

It is evident that a malicious application can interfere with other (benign) applications silently, neither being recognized by end user nor by the underlying OS, by simply ex-

\footnotetext{
$1_{\text {https://developer.android.com/guide/topics/manifest/activity-element }}$
} 


\begin{tabular}{|c|c|c|c|c|c|}
\hline Solution & $\begin{array}{l}\text { Vulnerability } \\
\text { Discovery }\end{array}$ & Protection & $\begin{array}{c}\text { Implementation } \\
\text { Layer }\end{array}$ & Prerequisites & Availability \\
\hline Ren et al. [81] & Yes & Exploitation & Application & - & No \\
\hline Wang et al. [89] & Yes & Exploitation & Application & - & No \\
\hline Ren et al. [79] & Yes & Threat Identification & - & - & No \\
\hline Xiao et al. [93] & Yes & Detection & Application & - & No \\
\hline Ren et al. [80] & No & Prevention & Runtime & Device to be rooted & No \\
\hline Lee et al. [55] & No & Detection & Application & - & Yes \\
\hline Liu et al. [61] & Yes & Detection & Application & Bouncer & Yes \\
\hline Wu et al. [92] & No & Prevention & Operating System & OS modification & No \\
\hline Hwang et al. [48] & No & Prevention & Operating System & OS modification & No \\
\hline TaskAuth [66] & No & Prevention & Operating System & OS modification & Yes \\
\hline Anactijax [66] & Yes & Detection / Exploitation & Application & - & Yes \\
\hline
\end{tabular}

Table 5.1 Task and Activity Hijacking Related Works Comparison.

tracting the required information from the targeted application's manifest. In particular, a malicious application can inject an activity into another application's task stack by pointing its taskAffinity attribute to the target (legitimate) application's package name and the launchMode attribute being equals to singleTask, or equivalently the malicious activity is initiated with the intent flag FLAG_ACTIVITY_NEW_TASK set. The same result, against a targeted app, can also occur with various combinations of flags and attributes i.e., by using allowTaskReparenting attribute that the malicious application 'manipulates' in order to accomplish hijacking attacks. Similarly, benign applications that expose their activities to other applications are vulnerable against the same type of attacks. Figure 5.1 shows how a malicious application can accomplish a hijacking attack against any app on the left side. On the right side, a slightly different case is illustrated where an application explicitly defines a taskAffinity for an activity that can be manipulated by malicious applications. 

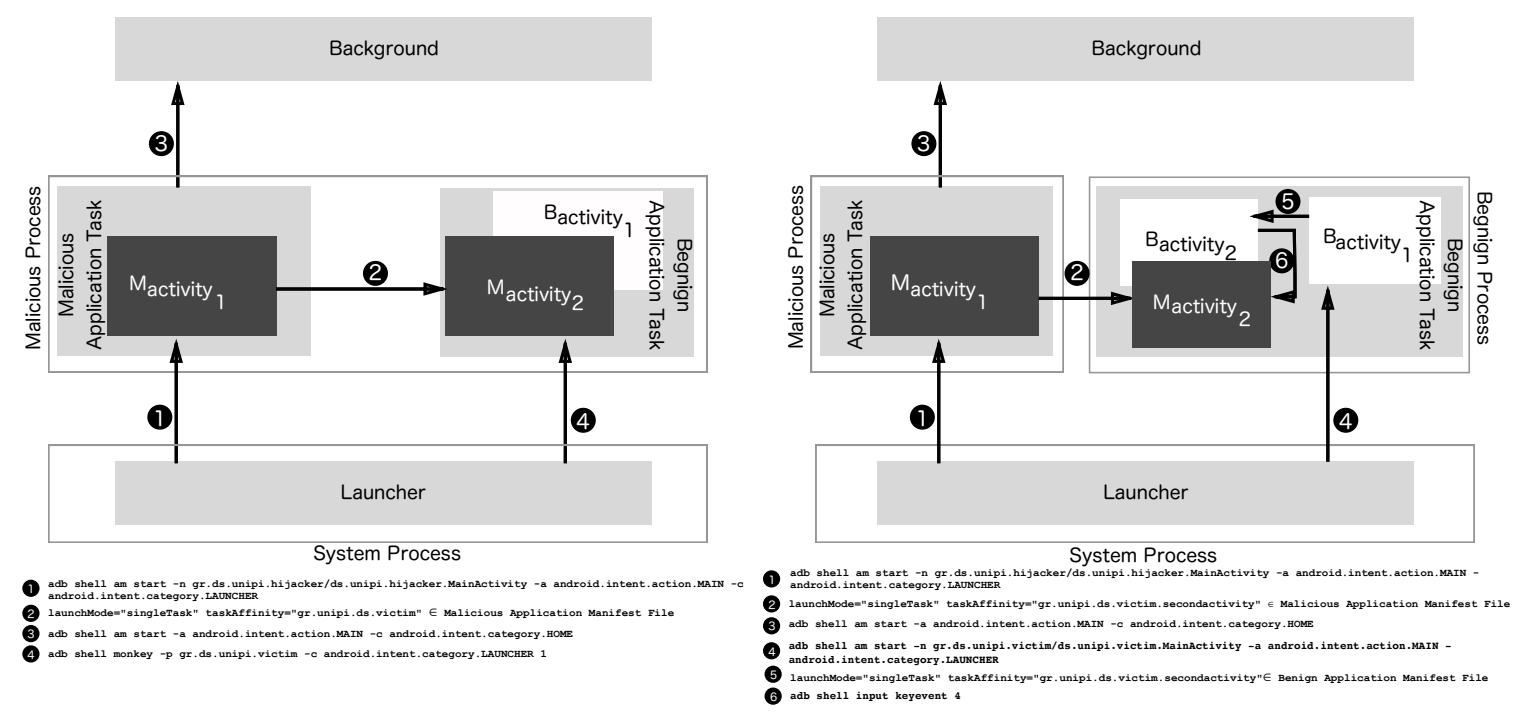

Figure 5.1 Hijacking Execution Flows.

\subsection{Android Task and Activity Hijacking Attacks}

Various researchers have attempted to address task [55, 48, 93, 61, 92] and activity $[81,89,79,80]$ hijacking vulnerabilities, through various different approaches. Currently, some of them have become obsolete [81, 89] due to Android framework's revisions, others require device 'rooting' [80] to enforce controls, others demand end users' intervention [93] assuming that their capable of recognizing malicious activities, others suffers from wellknown limitations of static analysis in Android applications (for instance: disregard of implicit intents invocations) [55, 48]. Orthogonal solutions, such as [61], propose the deployment of additional static and dynamic analysis controls at vetting process, whereas authors in [92] introduce a system level mechanism, by modifying the underlying OS, in order to inform end users for possible activity hijacking attacks. In [48] a reformation of the current Android application development framework is required for introducing a signature based mechanism to protect Android applications against task hijacking attacks.

\subsubsection{Exploitation and Detection Mechanisms}

Ren et al. [81] demonstrate a phishing attack against Android activities that require end user's input such as username and password. To do so, the proposed attack assumes that a malicious service is constantly running in the background in order to remain 'hidden'. Whenever the initialization of the target process is detected a malicious fake activity appears, impersonating the original one, by deceiving the end user and steal her/his credentials. In 
the same direction, Wang et al. [89] develop an application named ActivityHijacker that mimic legitimate application's activities and perform phishing attacks. Authors demonstrate the applicability of their approach by performing activity phishing attacks against twenty two (22) banking applications, and introduce a static analysis tool, named DroidChain, that can identify malicious application's behaviors based on legitimate application's invocations sequences. However, security flaws identified both by Ren et al. [81] and Wang et al. [89] are no longer applicable due to system modifications at Android's latest versions.

In another work, Ren et al. [79] was the first that systematically study and address the activity hijacking vulnerabilities in Android OS. Their research reveals different types of attacks against activities such as (a) phishing, (b) denial of service, (c) end user monitoring and (d) spoofing. Authors analyze 6.8 million applications to highlight the importance of the identified vulnerabilities in the Android framework and demonstrate that various applications attributes might be manipulated for achieving activity hijacking attacks, and introduce a theoretical mechanism able to circumvent activity hijacking in the Android framework, in which it is recommended that the taskAffinity attribute should be scrutinised by Google Bouncer [74].

Lee et al. [55] in their work demonstrate how a malicious activity can be injected in a legitimate (target) application's task stack. To do so, a malicious application should have define the target application's package name to the taskAffinity attribute in its manifest file, while the corresponding malicious activity is "initiated" as a new task with the intent flag FLAG_ACTIVITY_NEW_TASK set. Authors considering the different combinations of launchMode and task related intent flags demonstrate that such an attack can be performed in more than two hundred ways, and deploy a static analysis tool named AIDetector ${ }^{2}$ to detect vulnerable applications by correlating information extracted from application's manifest and its disassembled code. The AIDetector reports possible injection cases whenever an application declares activities that contain different taskAffinity attribute from their (original) package name, combined with attributes such as the lunchMode. However, AIDetector does not consider implicit intents or flows involving built-in method calls or static fields, and might report false positives combinations as it does not trigger the execution of the activity. Authors extend their approach in [48] where they introduce a signature-based defense mechanism that requires the modification of: (a) Android application's structure (by adding new tags into activity attributes), (b) Android framework, and Google's vetting process to enforce their scheme. More precisely, authors introduce a signature-based activity access control mechanism based on policies that define each application's interactions with other services and applications. The policies are defined by application developers during the development

\footnotetext{
${ }^{2}$ https://github.com/SunghoLee/AIDetector
} 
phase. So, whenever a developer needs to modify an application interaction policy, all the relevant applications should be recompiled and republished to the Google Play store.

Xiao et al. [93] study activity injection attacks by combining, for both the malicious and benign activities, (a) the allowTaskReparenting, (b) the taskAffinity attributes (c) the different launch Intent flags and (d) the sequences of launching events. They developed an activity hijacking vulnerability tool that follows a similar approach with Anactijax. It combines activity attributes and intent flags to generate pairs of malicious and benign applications that are executed under various scenarios to identify possible task interference combinations. In this way, the authors demonstrate four task interference proof-of-concept attacks: (a) UI Phishing, (b) Activity-in-the-middle, (c) Gallery Stealing and (d) Screen Shot Capturing. In order to minimise the attack surface and protect end users against such a threat, the authors introduce a task interference detection application named Task Interference Checking (TICK), to detect potential task interferences among applications. Although, TICK has no prevention capabilities. In fact, TICK scans either an application before installation or periodically a set of installed application to report possible activity hijacking vulnerabilities, without allowing any action to be taken against malicious activities.

Liu et al. [61] in their study propose a tool, named TDroid ${ }^{3}$, applicable to apps' distribution market in order to enhance the vetting [74] process. TDroid combines static and dynamic analysis to detect malicious applications that attempt to replace legitimate application's top activity. During the static analysis phase, TDroid transforms the application into runnable slices to check if they contain potentially activity related attacks. In the second phase of dynamic analysis, TDroid executes these slices on an Android phone or emulator and extracts possible malicious activities. However, such an approach does not consider: (a) the intercomponent communication (ICC) and reflection APIs, (b) the data dependencies through persistent storage that might result in partial hijacking attacks identification.

Lyvas et al. [66] developed a tool, named Anactijax (Android Activity Hijacking Examiner), capable of accomplishing activity hijacking attacks against other (target) applications, demonstrating through experiments how a malicious application functions in such a scenarios. Anactijax provides a framework for automatically generating a malicious application to accomplish activity hijacking attacks. This is achieved by retrofiring the malicious application generator with specific flags and attributes i.e., such info can be extracted from legitimate (target) application's manifest..

On one hand, Anactijax, similar to Xiao et al. [93], automatically calculates all the combinations of intent flags and <activity> attributes extracted from the Android Software Development Kit (SDK), aiming at generating malicious activities that can be injected against

\footnotetext{
$3_{\text {https }} / / /$ tdroidtool.github.io
} 
any benign application, employing a brute force approach. On the other hand, Anactijax has the capacity to generate activity injection use cases against real-world Android applications; a functionality that, no other current tool supports. As Figure 5.2 depicts, Anactijax for each combination of intent flag and <activity> attribute, Anactijax deploys a malicious application against a benign one (target), and deduce whether the malicious activity has been successfully pushed in the benign application's task by monitoring system's and application's $\operatorname{logs}$.

To demonstrate activity hijacking attacks an environment where a malicious and a benign application co-exist in an Android device, has been assumed. The malicious application runs in the background and launches attacks against any given (target) application considering the default settings i.e., the target application exports the main activity. In order to assess its accuracy and correctness, Anactijax has been deployed on a standard Android emulator version 9 with 2 GB of RAM and 1 Central Processing Unit (CPU), running on a machine with an Intel Core i5 1,3 GHz CPU and 8 GB RAM.

For testing purposes a customized benign application was developed, that contains a main activity which is by default exported and, alternatively, that it declares an explicit taskAffinity for an activity that Anactijax targets. In this context, Anactijax generates all the possible combinations considering different activity attributes with related intent flags. For instance, considering the use of 2 or 3 activity attributes with related intent flags, Anactijax creates 3744 uses cases to be executed against the target app, and reports 80 successful activity injection attacks. Table 5.2 provides and overview of the analysis of the tested use cases. Results suggest that successful hijacking attacks combine either activity's taskAffinity and launchMode attributes, or rely on FLAG_ACTIVITY_NEW_TASK intent flag.

The ability of Anactijax to produce malicious applications which can launch activity injection attacks against real-world applications, relies on its capacity to extract the appropriate features from the targeted APK as Figure 5.3 depicts. For any given real-world application, Anactijax extracts its manifest file, exports its package name, the main activity's name, and any other activity name with the taskAffinity value set. Note that the information in the manifest file is in clear text as it is used by Android OS to execute properly the application. The overall approach has been already described above.

In particular to demonstrate that a malicious application can take the control of a benign (target) one: case (a) (left side of Figure 5.1) during the initialization of the target application, Anactijax generates a hijacking application that contains a manifest with a taskAffinity attribute value equal to the target application's package name and adds the necessary Java code for the initialization of the malicious activity with the intent flag FLAG_ACTIVITY_NEW_TASK set in the malicious activity's source code, and afterwards it generates the malicious 
APK and case (b) (right side of Figure 5.1) while the user navigates to benign application activities Anactijax injects a malicious activity into the benign application in such a way that the malicious task is placed in the stack of the benign application and will be triggered when the user navigates back. To do so Anactijax searches if the given benign application code activities define explicitly the taskAffinity attribute. If this is the case it extracts the taskAffinity value and generates a hijacking application that contains a malicious activity with the taskAffinity attribute value equal to the victim activity's one (in the malicious applications Manifest file). Then it adds the necessary Java code for the initialization of the malicious activity with the intent flag FLAG_ACTIVITY_NEW_TASK set in the malicious activity's source code in order to be capable to be injected into target application's stack.

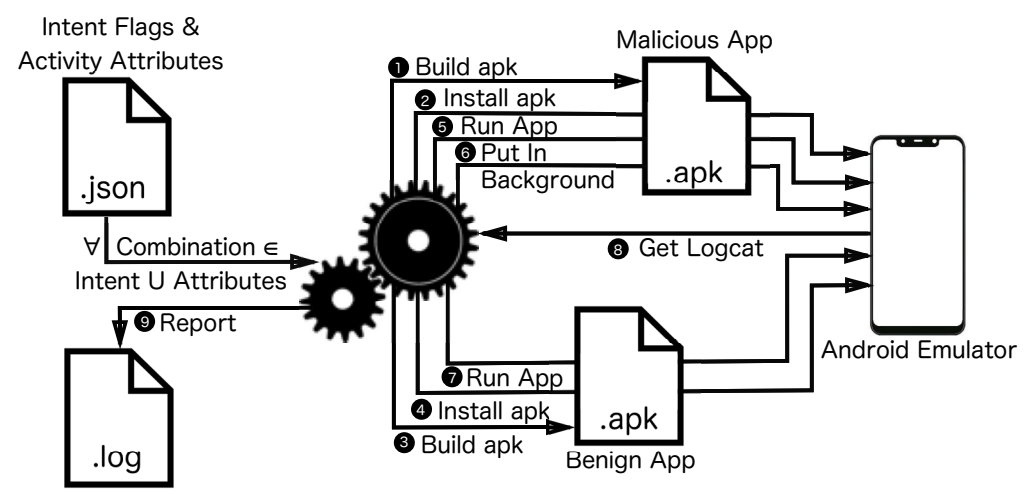

Figure 5.2 High-Level Design of Anactijax for Task and Activity Injection Related Activity Attributes and Intent Flag Exfiltration.

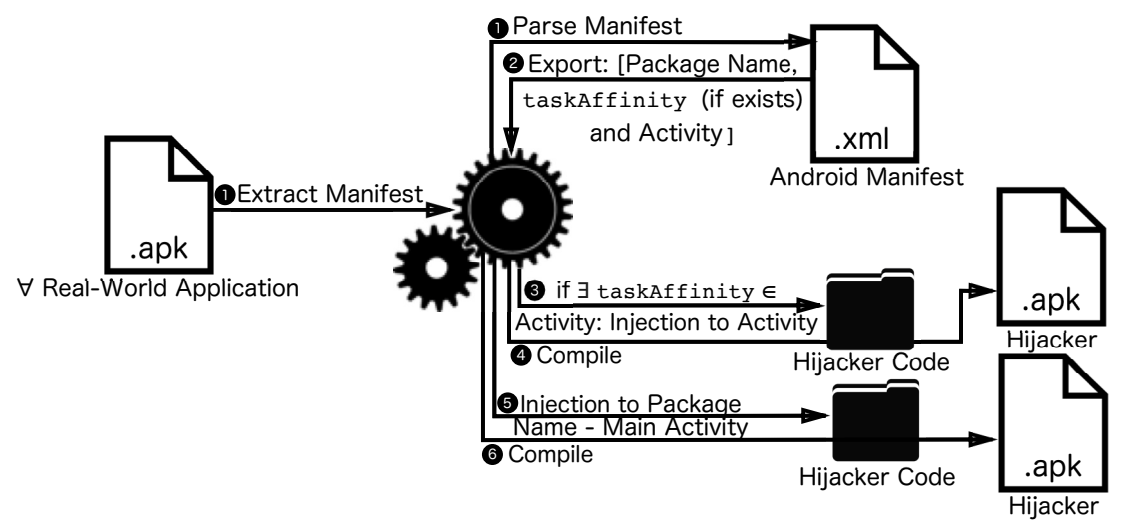

Figure 5.3 High-Level Design of Anactijax for Automated Hijacking Application Generation Against Real-World Applications. 


\subsubsection{Prevention Mechanisms}

Wu et al. [92] provided a detection service that alerts end users for possible password phishing attempts via activity hijacking attacks. The proposed system-level mechanism is limited only to inform users via pop-up windows for potential activity hijacking attacks whenever password fields are involved.

In an extended approach of Xiao et al. [55] a signature-based defense mechanism named SAAC was proposed in [48] that requires the modification of: (a) Android application's structure (by adding new tags into activity attributes), (b) Android framework, and Google's vetting process to enforce their scheme. More precisely, authors introduce a signature-based activity access control mechanism based on policies that define each application's interactions with other services and applications. The policies are defined by application developers during the development phase. So, whenever a developer needs to modify an application interaction policy, all the relevant applications should be recompiled and republished to the Google Play store.

Ren et al. [80] designed an application able to prevent Graphic User Interface (GUI) attacks against Android named WindowGuard, a solution that requires the device to be 'rooted' as it is deployed upon Xposed framework [34]. WindowGuard enforces an android window integrity mechanism (AWI) that provides the following enhancements into Android GUI system: (a) an activity session integrity, (b) access control for the free windows, and (c) safeguarding of focused activity transition. The effectiveness of WindowGuard has been evaluated over 12,060 applications, which $1 \%$ trigger warning for possible activity manipulation.

In order to prevent task and activities hijacking attacks and, at the same time, minimize Android's attack surface, it is proposed [66] to enhance the Android's task management mechanism by introducing a system level service, namely the TaskAuth, that authenticates the activity's source whenever it invokes other applications. In order for the proposed approach to be transparent, it leverages on application's signature verification scheme, used during application's initialization, by extending the ActivityManager system service. In this way, TaskAuth, does not require any intervention or application modification by end users or developers respectively.

More specifically, TaskAuth, verifies whether or not the identities of source and target activities match, as depicted in Figure 5.4. To do so, TaskAuth whenever an activity (source) invokes another one (target), TaskAuth scrutinizes source activity's taskAffinity and processName fields of aInfo object ${ }^{4}$ and checks if the source activity declares another application's package name in the taskAffinity field. If this is not the case, TaskAuth does not intervene and the execution is processed normally, otherwise it retrieves a PackageInfo

\footnotetext{
${ }^{4}$ belongs to the ActivityInfo class.
} 


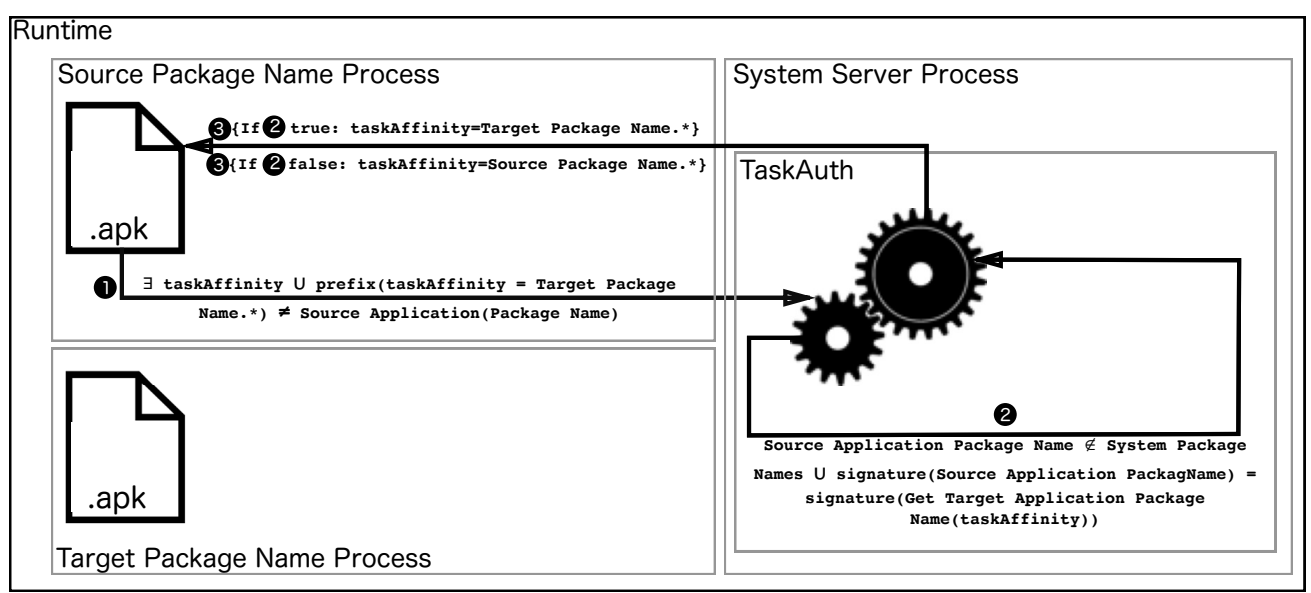

Figure 5.4 TaskAuth High Level Design.

object for both activities, by executing the getPackageInfo ( . . ), that among others contains the application's singing certificates and compares them to determine whether source and target activities packages belong to the same developer.

At this point it should be noted that TaskAuth considers legitimate the declaration of the taskAffinity pointing in another application's package name or in another application's package name, if both belong to the same developer, or the source activity belongs to a trusted organisation i.e., Google. Otherwise, TaskAuth classifies it as a task hijacking attempt and 'blocks' it by setting dynamically the taskAffinity of the malicious activity to the default value that is its package name. In this way, taskAffinity does not affect the application's execution at all. Of course, other policies could be retrofitted to the TaskAuth for covering additional needs.

\subsubsection{Empirical Analysis}

For evaluating [66] the capacity of Anactijax to generate malicious applications that can attack real-world applications as well as the ability of TaskAuth to prevent these attacks, 300 applications, were randomly downloaded after random sampling from a pull of 5.350 unique application titles crawled from Google Play ${ }^{5}$ covering the majority of all the provided application categories. From this initial application set, 52 were supporting only ARM architectures and another 34 applications could not be executed on the emulated environment and thus were excluded. Finally for 14 applications Anactijax was unable to extract features.

$5_{\text {https: //play.google.com/store }}$ 
For the remaining 200 applications (three applications on average from 43 different application categories such as games, social, finance, business etc.), we used an automated procedure in order to evaluate the functionality of Anactijax and the effectiveness of TaskAuth. More precisely, Anactijax was used to generate hijacking applications that target a specific real-world application. Then, with the aid of a standard Android emulator, we automatically executed the benign and the malicious applications, and by monitoring the results of dumpsys ${ }^{6}$ we were able to determine if the malicious application managed to inject the malicious activity into a benign application task or if the benign application task was injected into the malicious process.

For the 200 applications, Anactijax was able to generate 202 applications (requiring on average, $7.2 \mathrm{~s}$ for each one) from which 200 were targeting the given application's main activity with taskAffinity pointing to the targeted application's package name (benign task injection into the malicious process - case - a) and 2 malicious applications targeting benign activities with declared taskAffinity (malicious activity injection into the benign process - case - b).

After executing each malicious application against each targeted benign application, we identified two cases where the activity injection failed because the developers of the benign applications had defined their main activities with the flag launchMode set to a singleTask and their back button is used to exit directly the applications rather than navigating among activities. For the rest 198 cases (99\%), the malicious applications were able to accomplish successfully activity injection attacks.

At this point it is worth mentioning that 28 targeted applications had declared their main activity with the flag launchMode set to singleTop something that made them vulnerable to malicious activity injection into the benign process (case - b) rather than being vulnerable to benign task injection into the malicious process (case - a) which was the case for the remaining 170 applications.Finally, for 10 applications out of 28 , the malicious activity was injected on top of a splash screen main activity that instantly initiated other activities, a fact that caused the injected activity to remain invisible to the user.

TaskAuth was deployed on a modified Android framework version 9 on the standard Android emulator with $2 \mathrm{~GB}$ of RAM and $1 \mathrm{CPU}$ on a machine featuring Intel Core i5 1,3 GHz CPU and 8 GB RAM, and we have evaluated its effectiveness in terms of: (a) efficacy to detect hijacking attacks and (b) overhead introduced i.e., . how TaskAuth influences application performance.

\footnotetext{
${ }^{6}$ The adb shell dumpsys activity command shows the current state of running activities into a system along with their task records.
} 


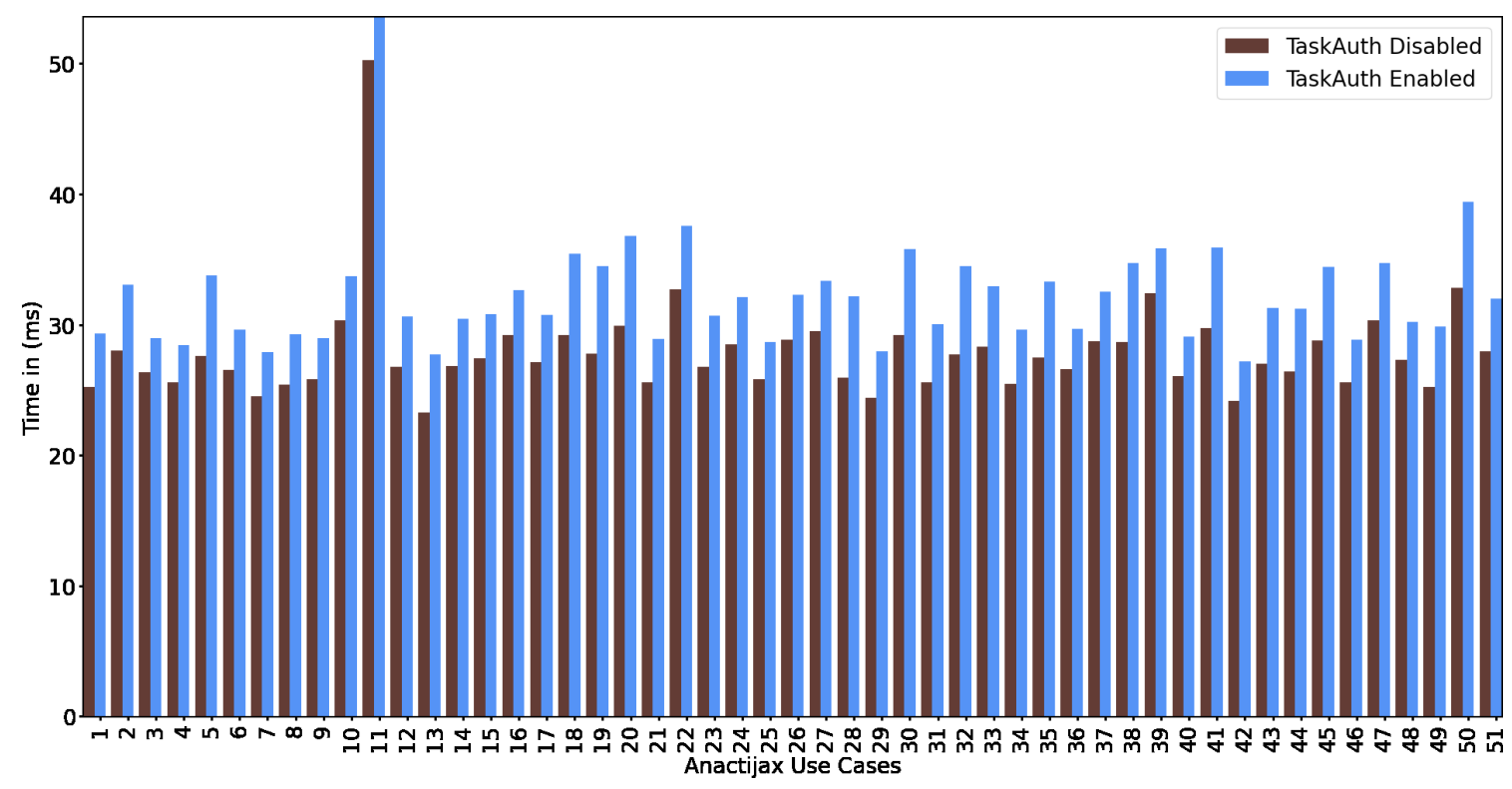

Figure 5.5 Overhead Introduced by TaskAuth to the (Default Exported) Main Activity With Default (Application Package Name) taskAffinity.

From evaluation standpoint, TaskAuth was tested against Anactijax were Anactijax has not reported any successful injection attack (from the initial 80 injection attacks) when TaskAuth is enforced. Table 5.3 overviews the results of the tests, where the malicious application $\left(M_{\text {process }}\right)$ was unable to inject a malicious activity $\left(M_{\text {activity }}\right)$ into the benign task $\left(B_{\text {task }}\right.$ or $B_{\text {process }}$ ), thus allowing the benign process ( $B_{\text {process }}$ ) to proceed with its normal execution in all identified activity injection cases.

As far as the TaskAuth processing overhead is concerned, results have indicated that it has caused a negligible increase of the execution time i.e., the maximum observed execution time was as little as $7 \mathrm{~ms}$, with a minimum of $2.6 \mathrm{~ms}$, while it requires on average $4.3 \mathrm{~ms}$ to detect whether an activity is malicious or not as Figure 5.5 and 5.6 show.

In the same direction, to assess TaskAuth efficacy to protect also real-world applications, the Anactijax malicious generated applications were reused and repeated the hijacking attacks while the TaskAuth was in place. The results demonstrate that TaskAuth managed to prevent all the hijacking attack attempts and also that its performance, in terms of the overhead introduced, is similar to the demo use case (see previous paragraphs). In particular, the overhead introduced is on average $4.8 \mathrm{~ms}$, with a minimum of $2.6 \mathrm{~ms}$ and a maximum of 6.9 ms as Figure 5.7 illustrates. 


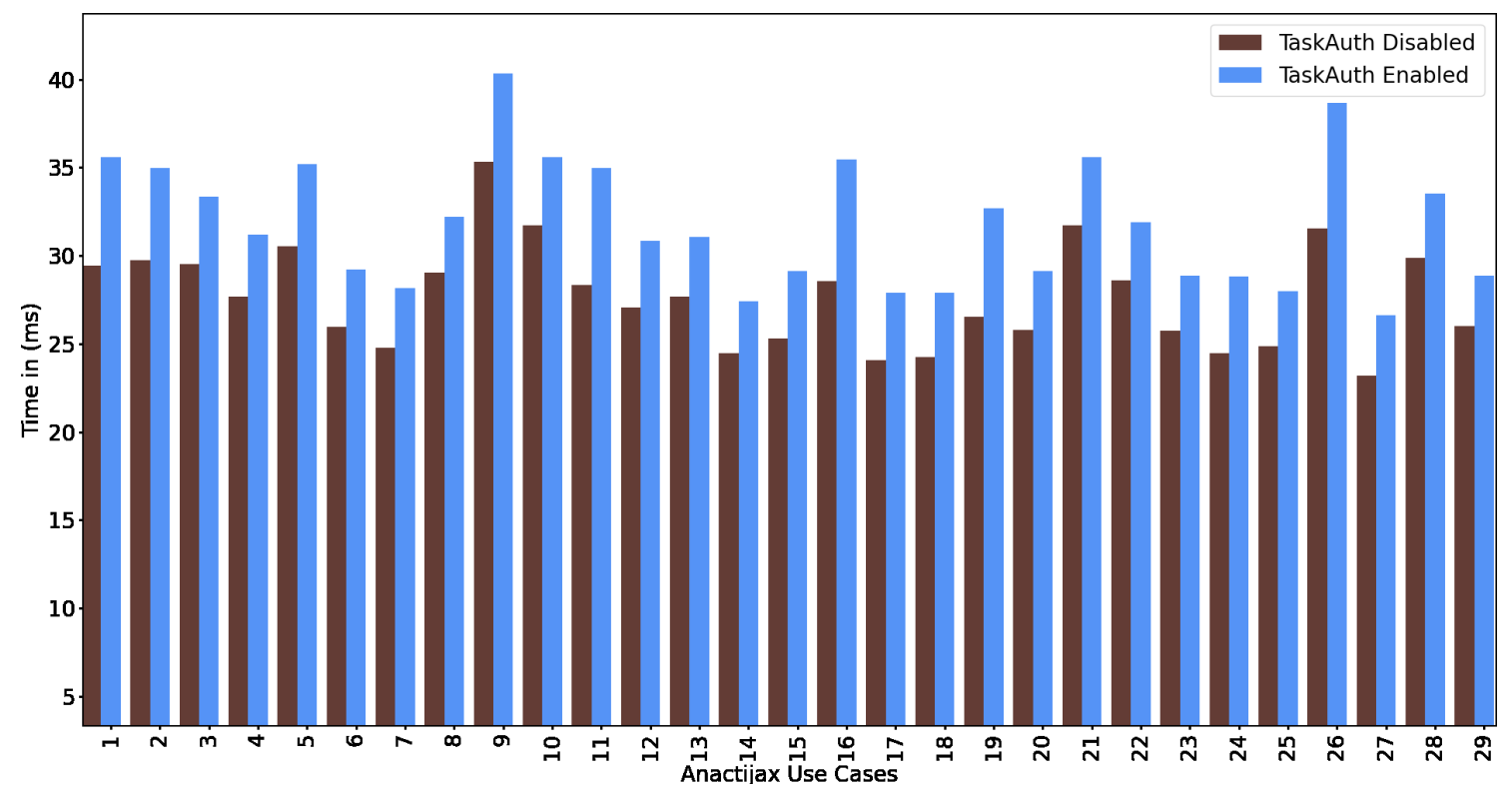

Figure 5.6 Overhead Introduced by TaskAuth for Vulnerable Applications

With Activities That Declare Explicitly taskAffinity Other Than the Default (Application Package Name).

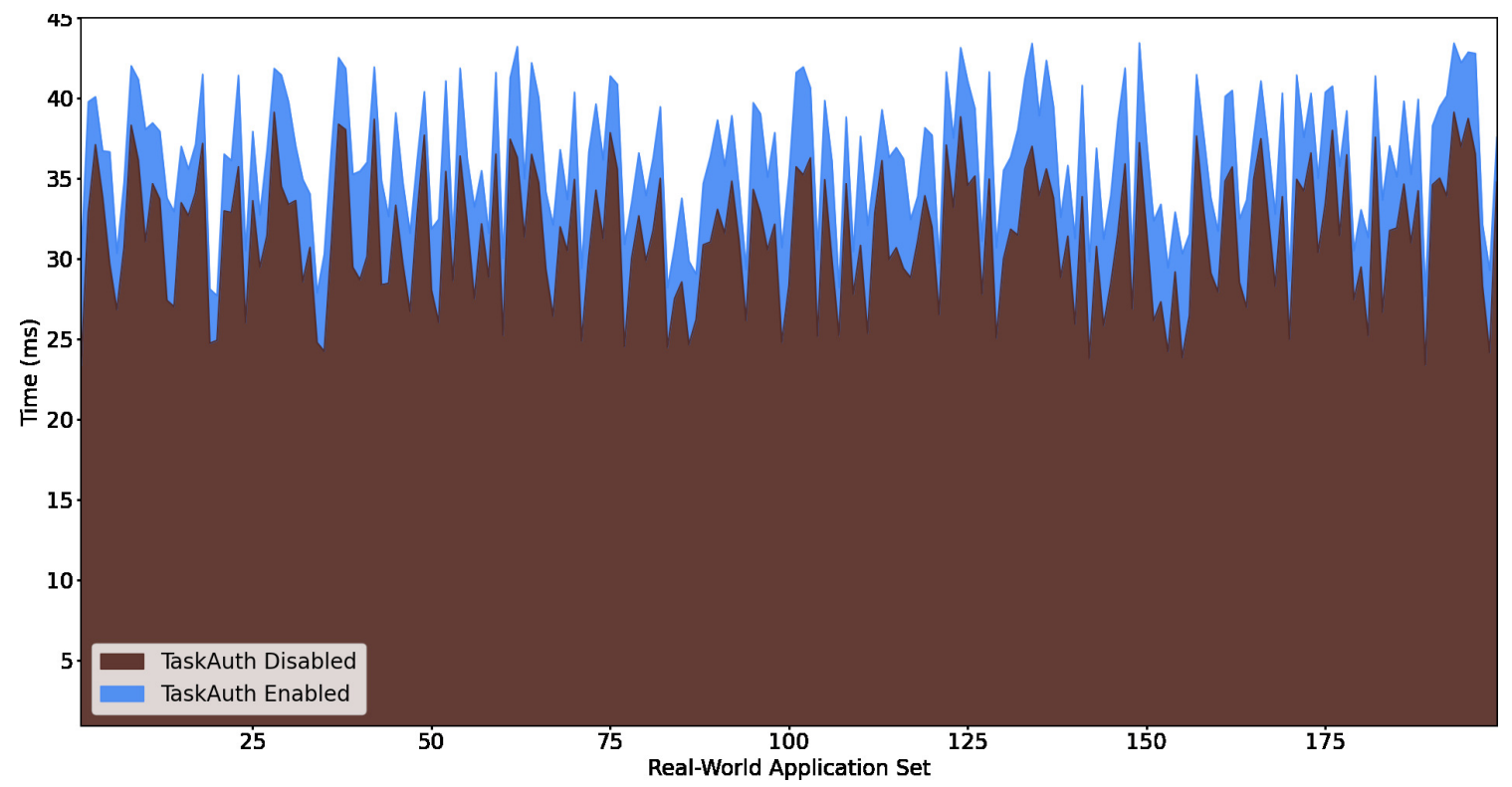

Figure 5.7 Overhead Introduced by TaskAuth For Each Real-World Application Used in the Evaluation (Total Two Hundred Applications). 


\begin{tabular}{|c|c|c|c|c|c|}
\hline \multirow[t]{2}{*}{$\#$} & \multicolumn{2}{|l|}{ Malicious Application } & \multicolumn{2}{|c|}{ Benign Application } & \multirow[t]{2}{*}{ Impact } \\
\hline & Activity Attributes & Intent Flags & Activity Attributes & Intent Flags & \\
\hline 1 & $\begin{array}{l}\text { launchMode="singleTask" } \\
\text { taskAffinity= } \\
\text { "gr.unipi.ds.victim" }\end{array}$ & - & - & - & $\begin{array}{c}M_{\text {activity }} \in B_{\text {task }} \mid \\
B_{\text {task }} \in M_{\text {process }}\end{array}$ \\
\hline 2 & $\begin{array}{l}\text { launchMode="singleTask" } \\
\text { taskAffinity= } \\
\text { "gr.unipi.ds.victim } \\
\text { allowTaskReparenting="true" }\end{array}$ & - & - & - & $\begin{array}{c}M_{\text {activity }} \in B_{\text {task }} \mid \\
B_{\text {task }} \in M_{\text {process }}\end{array}$ \\
\hline 3 & $\begin{array}{l}\text { taskAffinity= } \\
\text { "gr.unipi.ds.victim }\end{array}$ & FLAG_ACTIVITY_NEW_TASK & - & - & $\begin{array}{c}M_{\text {activity }} \in B_{\text {task }} \mid \\
B_{\text {task }} \in M_{\text {process }}\end{array}$ \\
\hline 4 & $\begin{array}{l}\text { allowTaskReparenting="true" } \\
\text { taskAffinity= } \\
\text { "gr.unipi.ds.victim }\end{array}$ & FLAG_ACTIVITY_NEW_TASK & - & - & $\begin{array}{c}M_{\text {activity }} \in B_{\text {task }} \mid \\
B_{\text {task }} \in M_{\text {process }}\end{array}$ \\
\hline 5 & $\begin{array}{l}\text { allowTaskReparenting="true" } \\
\text { taskAffinity= } \\
\text { "gr.unipi.ds.victim } \\
\text {.secondactivity" }\end{array}$ & FLAG_ACTIVITY_NEW_TASK & $\begin{array}{l}\text { taskAffinity }= \\
\text { "gr.unipi.ds.victim } \\
\text {.secondactivity" }\end{array}$ & FLAG_ACTIVITY_NEW_TASK & $\begin{array}{c}M_{\text {activity }} \in B_{\text {task }} \mid \\
B_{\text {task }} \in B_{\text {process }}\end{array}$ \\
\hline 6 & $\begin{array}{l}\text { taskAffinity= } \\
\text { "gr.unipi.ds.victim } \\
\text {.secondactivity" }\end{array}$ & FLAG_ACTIVITY_NEW_TASK & $\begin{array}{l}\text { taskAffinity= } \\
\text { "gr.unipi.ds.victim } \\
\text {.secondactivity" }\end{array}$ & FLAG_ACTIVITY_NEW_TASK & $\begin{array}{c}M_{\text {activity }} \in B_{\text {task }} \mid \\
B_{\text {task }} \in B_{\text {process }}\end{array}$ \\
\hline 7 & $\begin{array}{l}\text { launchMode="singleTask" } \\
\text { taskAffinity= } \\
\text { "gr.unipi.ds.victim } \\
\text {.secondactivity" }\end{array}$ & - & $\begin{array}{l}\text { taskAffinity }= \\
\text { "gr.unipi.ds.victim } \\
\text {.secondactivity" }\end{array}$ & FLAG_ACTIVITY_NEW_TASK & $\begin{array}{c}M_{\text {activity }} \in B_{\text {task }} \mid \\
B_{\text {task }} \in B_{\text {process }}\end{array}$ \\
\hline 8 & $\begin{array}{l}\text { launchMode="singleTask" } \\
\text { taskAffinity= } \\
\text { "gr.unipi.ds.victim } \\
\text {.secondactivity" } \\
\text { allowTaskReparenting="true" }\end{array}$ & - & $\begin{array}{l}\text { taskAffinity= } \\
\text { "gr.unipi.ds.victim } \\
\text {.secondactivity" }\end{array}$ & FLAG_ACTIVITY_NEW_TASK & $\begin{array}{c}M_{\text {activity }} \in B_{\text {task }} \mid \\
B_{\text {task }} \in B_{\text {process }}\end{array}$ \\
\hline 9 & $\begin{array}{l}\text { allowTaskReparenting="true" } \\
\text { taskAffinity= } \\
\text { "gr.unipi.ds.victim } \\
\text {.secondactivity" }\end{array}$ & FLAG_ACTIVITY_NEW_TASK & $\begin{array}{l}\text { launchMode="singleTask" } \\
\text { taskAffinity= } \\
\text { "gr.unipi.ds.victim } \\
\text {.secondactivity" }\end{array}$ & - & $\begin{array}{c}M_{\text {activity }} \in B_{\text {task }} \mid \\
B_{\text {task }} \in B_{\text {process }}\end{array}$ \\
\hline 10 & $\begin{array}{l}\text { taskAffinity= } \\
\text { "gr.unipi.ds.victim } \\
\text {.secondactivity" }\end{array}$ & FLAG_ACTIVITY_NEW_TASK & $\begin{array}{l}\text { launchMode="singleTask" } \\
\text { taskAffinity= } \\
\text { "gr.unipi.ds.victim } \\
\text { secondactivity" }\end{array}$ & - & $\begin{array}{c}M_{\text {activity }} \in B_{\text {task }} \mid \\
B_{\text {task }} \in B_{\text {process }}\end{array}$ \\
\hline 11 & $\begin{array}{l}\text { launchMode="singleTask" } \\
\text { taskAffinity= } \\
\text { "gr.unipi.ds.victim } \\
\text {.secondactivity" }\end{array}$ & - & $\begin{array}{l}\text { launchMode="singleTask" } \\
\text { taskAffinity= } \\
\text { "gr.unipi.ds.victim } \\
\text {.secondactivity" }\end{array}$ & - & $\begin{array}{c}M_{\text {activity }} \in B_{\text {task }} \mid \\
B_{\text {task }} \in B_{\text {process }}\end{array}$ \\
\hline 12 & $\begin{array}{l}\text { launchMode="singleTask" } \\
\text { taskAffinity= } \\
\text { "gr.unipi.ds.victim } \\
\text {.secondactivity" } \\
\text { allowTaskReparenting="true" }\end{array}$ & - & $\begin{array}{l}\text { launchMode="singleTask" } \\
\text { taskAffinity= } \\
\text { "gr.unipi.ds.victim } \\
\text { secondactivity" }\end{array}$ & - & $\begin{array}{c}M_{\text {activity }} \in B_{\text {task }} \mid \\
B_{\text {task }} \in B_{\text {process }}\end{array}$ \\
\hline
\end{tabular}

Table 5.2 Anactijax Has Successfully Identified Hijacking Combinations of Activity Attributes and Intent Flags. 


\begin{tabular}{|c|c|c|c|c|c|}
\hline \multirow[t]{2}{*}{$\#$} & \multicolumn{2}{|l|}{ Malicious Application } & \multicolumn{2}{|c|}{ Benign Application } & \multirow[t]{2}{*}{ Impact } \\
\hline & Activity Attributes & Intent Flags & Activity Attributes & Intent Flags & \\
\hline 1 & $\begin{array}{l}\text { launchMode="singleTask" } \\
\text { taskAffinity= } \\
\text { "gr.unipi.ds.victim" }\end{array}$ & - & - & - & $\begin{array}{l}M_{\text {activity }} \in M_{\text {task }} \in M_{\text {process }}, \\
B_{\text {activity }} \in B_{\text {task }} \in B_{\text {process }}\end{array}$ \\
\hline 2 & $\begin{array}{l}\text { launchMode="singleTask" } \\
\text { taskAffinity= } \\
\text { "gr.unipi.ds.victim } \\
\text { allowTaskReparenting="true" }\end{array}$ & - & - & - & $\begin{array}{l}M_{\text {activity }} \in M_{\text {task }} \in M_{\text {process }}, \\
B_{\text {activity }} \in B_{\text {task }} \in B_{\text {process }}\end{array}$ \\
\hline 3 & $\begin{array}{l}\text { taskAffinity= } \\
\text { "gr.unipi.ds.victim }\end{array}$ & FLAG_ACTIVITY_NEW_TASK & - & - & $\begin{array}{l}M_{\text {activity }} \in M_{\text {task }} \in M_{\text {process }} \\
B_{\text {activity }} \in B_{\text {task }} \in B_{\text {process }}\end{array}$ \\
\hline 4 & $\begin{array}{l}\text { allowTaskReparenting="true" } \\
\text { taskAffinity= } \\
\text { "gr.unipi.ds.victim }\end{array}$ & FLAG_ACTIVITY_NEW_TASK & - & - & $\begin{array}{l}M_{\text {activity }} \in M_{\text {task }} \in M_{\text {process }}, \\
B_{\text {activity }} \in B_{\text {task }} \in B_{\text {process }}\end{array}$ \\
\hline 5 & $\begin{array}{l}\text { allowTaskReparenting="true" } \\
\text { taskAffinity= } \\
\text { "gr.unipi.ds.victim } \\
\text {.secondactivity" }\end{array}$ & FLAG_ACTIVITY_NEW_TASK & $\begin{array}{l}\text { taskAffinity= } \\
\text { "gr.unipi.ds.victim } \\
\text {.secondactivity" }\end{array}$ & FLAG_ACTIVITY_NEW_TASK & $\begin{array}{l}M_{\text {activity }} \in M_{\text {task }} \in M_{\text {process }}, \\
B_{\text {activity }} \in B_{\text {task }} \in B_{\text {process }}\end{array}$ \\
\hline 6 & $\begin{array}{l}\text { taskAffinity= } \\
\text { "gr.unipi.ds.victim } \\
\text { secondactivity" }\end{array}$ & FLAG_ACTIVITY_NEW_TASK & $\begin{array}{l}\text { taskAffinity= } \\
\text { "gr.unipi.ds.victim } \\
\text {.secondactivity" }\end{array}$ & FLAG_ACTIVITY_NEW_TASK & $\begin{array}{l}M_{\text {activity }} \in M_{\text {task }} \in M_{\text {process }}, \\
B_{\text {activity }} \in B_{\text {task }} \in B_{\text {process }}\end{array}$ \\
\hline 7 & $\begin{array}{l}\text { launchMode="singleTask" } \\
\text { taskAffinity= } \\
\text { "gr.unipi.ds.victim } \\
\text {.secondactivity" }\end{array}$ & - & $\begin{array}{l}\text { taskAffinity= } \\
\text { "gr.unipi.ds.victim } \\
\text {.secondactivity" }\end{array}$ & FLAG_ACTIVITY_NEW_TASK & $\begin{array}{l}M_{\text {activity }} \in M_{\text {task }} \in M_{\text {process }} \\
B_{\text {activity }} \in B_{\text {task }} \in B_{\text {process }}\end{array}$ \\
\hline 8 & $\begin{array}{l}\text { launchMode="singleTask" } \\
\text { taskAffinity= } \\
\text { "gr.unipi.ds.victim } \\
\text {.secondactivity" } \\
\text { allowTaskReparenting="true" }\end{array}$ & - & $\begin{array}{l}\text { taskAffinity= } \\
\text { "gr.unipi.ds.victim } \\
\text {.secondactivity" }\end{array}$ & FLAG_ACTIVITY_NEW_TASK & $\begin{array}{l}M_{\text {activity }} \in M_{\text {task }} \in M_{\text {process }}, \\
B_{\text {activity }} \in B_{\text {task }} \in B_{\text {process }}\end{array}$ \\
\hline 9 & $\begin{array}{l}\text { allowTaskReparenting="true" } \\
\text { taskAffinity= } \\
\text { "gr.unipi.ds.victim } \\
\text {.secondactivity" }\end{array}$ & FLAG_ACTIVITY_NEW_TASK & $\begin{array}{l}\text { launchMode="singleTask" } \\
\text { taskAffinity= } \\
\text { "gr.unipi.ds.victim } \\
\text {.secondactivity" }\end{array}$ & - & $\begin{array}{l}M_{\text {activity }} \in M_{\text {task }} \in M_{\text {process }}, \\
B_{\text {activity }} \in B_{\text {task }} \in B_{\text {process }}\end{array}$ \\
\hline 10 & $\begin{array}{l}\text { taskAffinity= } \\
\text { "gr.unipi.ds.victim } \\
\text {.secondactivity" }\end{array}$ & FLAG_ACTIVITY_NEW_TASK & $\begin{array}{l}\text { launchMode="singleTask" } \\
\text { taskAffinity= } \\
\text { "gr.unipi.ds.victim } \\
\text {.secondactivity" }\end{array}$ & - & $\begin{array}{l}M_{\text {activity }} \in M_{\text {task }} \in M_{\text {process }}, \\
B_{\text {activity }} \in B_{\text {task }} \in B_{\text {process }}\end{array}$ \\
\hline 11 & $\begin{array}{l}\text { launchMode="singleTask" } \\
\text { taskAffinity= } \\
\text { "gr.unipi.ds.victim } \\
\text {.secondactivity" }\end{array}$ & - & $\begin{array}{l}\text { launchMode="singleTask" } \\
\text { taskAffinity= } \\
\text { "gr.unipi.ds.victim } \\
\text {.secondactivity" }\end{array}$ & - & $\begin{array}{l}M_{\text {activity }} \in M_{\text {task }} \in M_{\text {process }}, \\
B_{\text {activity }} \in B_{\text {task }} \in B_{\text {process }}\end{array}$ \\
\hline 12 & $\begin{array}{l}\text { launchMode="singleTask" } \\
\text { taskAffinity= } \\
\text { "gr.unipi.ds.victim } \\
\text {.secondactivity" } \\
\text { allowTaskReparenting="true" }\end{array}$ & - & $\begin{array}{l}\text { launchMode="singleTask" } \\
\text { taskAffinity= } \\
\text { "gr.unipi.ds.victim } \\
\text {.secondactivity" }\end{array}$ & - & $\begin{array}{l}M_{\text {activity }} \in M_{\text {task }} \in M_{\text {process }}, \\
B_{\text {activity }} \in B_{\text {task }} \in B_{\text {process }}\end{array}$ \\
\hline
\end{tabular}

Table 5.3 Execution Prevention of the Identified Activity Hijacking Use Cases of Anactijax after the Enforcement of TaskAuth. 


\section{Chapter 6}

\section{Android Intent Handling}

The Android framework offers to application components (from the same or different process) the option of interaction (send/receive or exchange data) via intents which are at a higher-level of abstraction executed over the Binder (as already mentioned in Subsection 3.1.3). Figure 6.1 depicts the control flow of an activity to activity communication between different applications through implicit intents. In detail, in the case where an application requests from some other component to take an action (i.e., open a document, through some available user application) it employs an implicit intent call, as it is not aware which application can handle such a request. Afterwards, the execution flow is transferred to the activity task manager service (ATMS) which is responsible for delivering it to the application that is able to handle the implicit intent request; such applications are those that contain the corresponding intent-filter, and normally the user is the one selecting it. Finally, ATMS delivers the implicit intent to the application (selected by the user) through the Android framework.

\subsection{Threats of Android Inter-Process Communication}

Inter-Process Communication (IPC) or Inter-Component Communication (ICC) in Android platform is performed over Intent message objects. Activity's implicit intents are used whenever a new activity is initialized with a 'description' of an action (A.2) rather than defining explicitly (A.4) a component to handle it, while simultaneously might be used to exchange data between different processes. This exchange mechanism might arises security and privacy implications since not only the source activity is unaware of the component that will manage it, but also the destination activity does not know the origin of the received intent driving either to intent redirection or malicious activity launch and intent hijacking attacks. On one side, the former category concerns cases where legitimate applications' publicly available exported components (i.e., activities) are exploited by malicious one to send malevolent data 


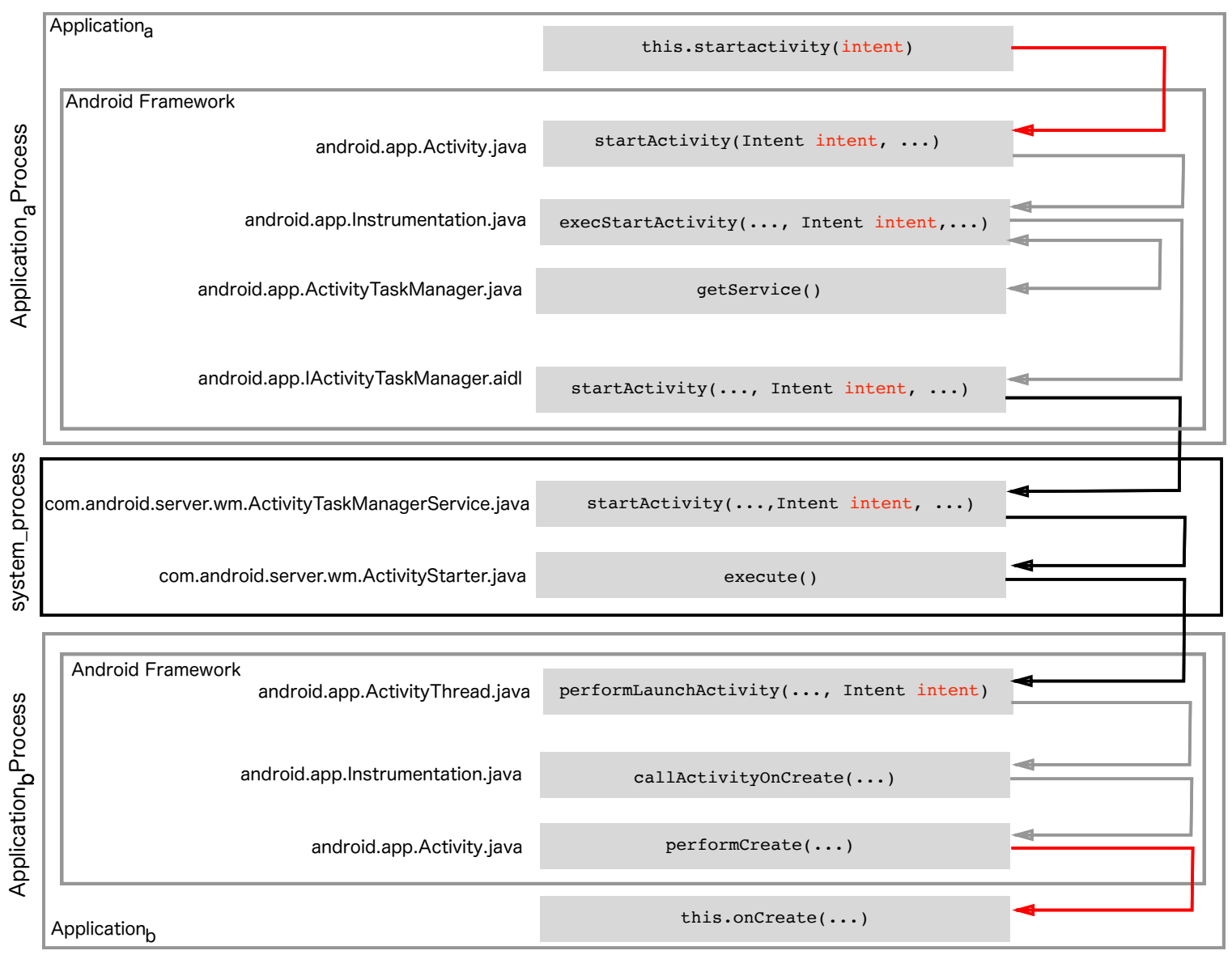

Figure 6.1 Android (Version 11) Activity Handling.

to other legitimate components and services, which might perform sensitive operations upon them, considering the data as trusted due to the fact that a well known source sent them. On the other side, the latest threat is related to malicious applications that define intent filters to intercept a legitimate application's transmitted data and gain unauthorized access to otherwise private data.

Currently, various research works attempt to address these security flaws using different approaches ranging from (a) static analysis detection tools [85, 37] to (b) system-level enhancements to prevent such attacks $[88,95]$ and minimize the OS's attack surface. Though their effectiveness is unquestionable, some of them suffer [85, 37] from the common limitations of static analysis approaches, while others that require the modification of the underlying OS, cannot be re-configured [88] dynamically, or expose intents beyond the interaction processes [95]. 
The comparison among the proposed methods regarding the Android intent redirection or malicious activity launch and intent hijacking, detection, and prevention mechanisms is listed in Table 6.1

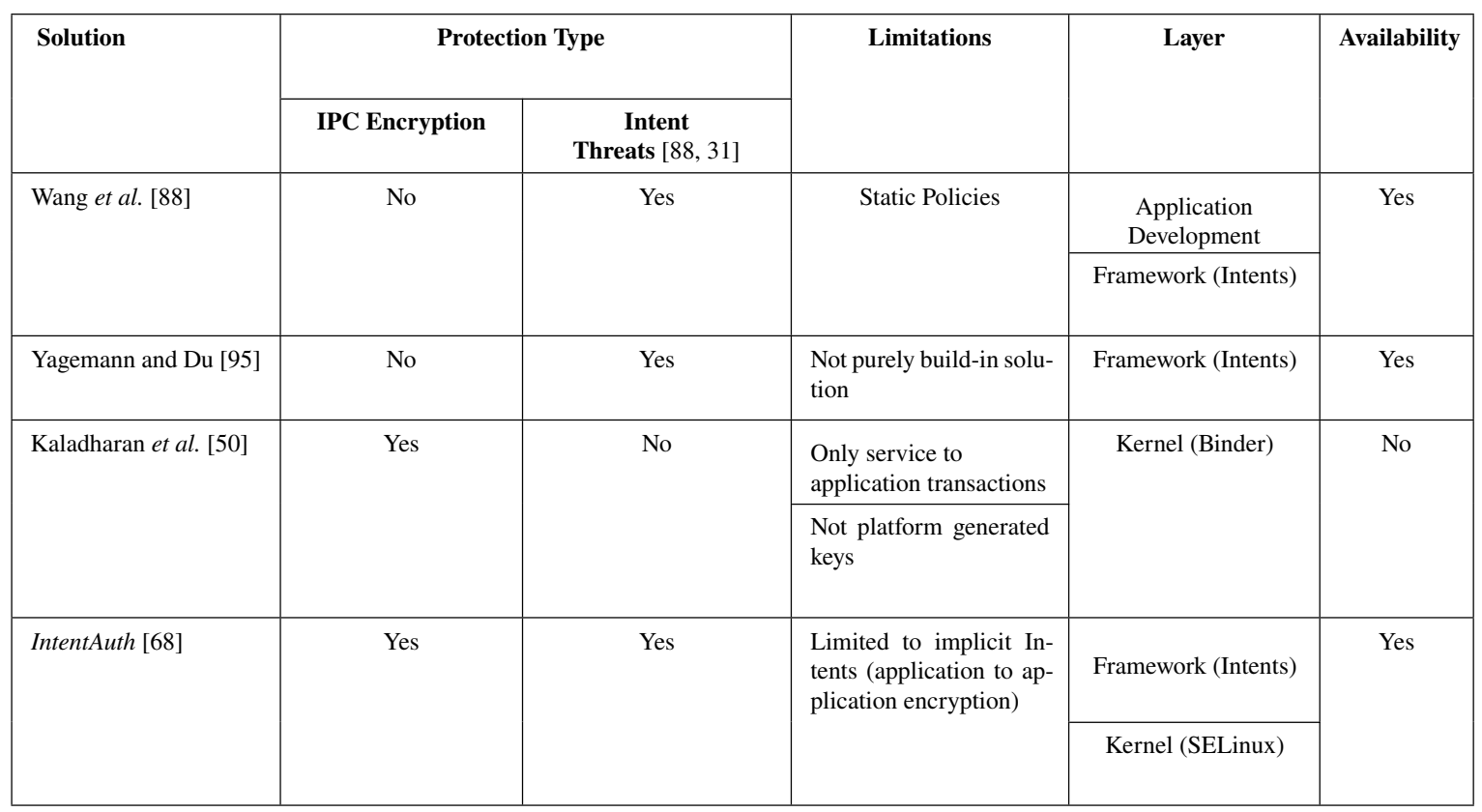

Table 6.1 Android Inter-Process Related Threat Prevention Mechanisms Comparison.

\subsubsection{Malicious Activity Launch and Intent Hijacking}

According to Chin et al. [31] a benign application that exports components (i.e., activities) to other applications may be manipulated by a malicious application that performs malicious activity launch attacks. In this case, a malicious application may corrupt the targeted application's storage by sending rogue data or by forcing the execution of specific components which return private or other sensitive data; an action based on the fact that the application receiving intents may not be able to verify the applications using its exported components. This type of attacks is primarily linked with design errors [49] by the application developers, who define activities with public access (public component) that perform sensitive operations over received data or return sensitive information upon their invocation without any restriction, ignoring the recommendations ${ }^{1}$ and the best practices that suggest that any activity that performs sensitive operations should be limited to invocations by activities inside the scope of the application they belong. Additionally, the platform allows activities from different applications to securely interact if they are protected through signature-level permissions

\footnotetext{
$1_{\text {https }} / / /$ cwe.mitre.org/data/definitions/926.html
} 


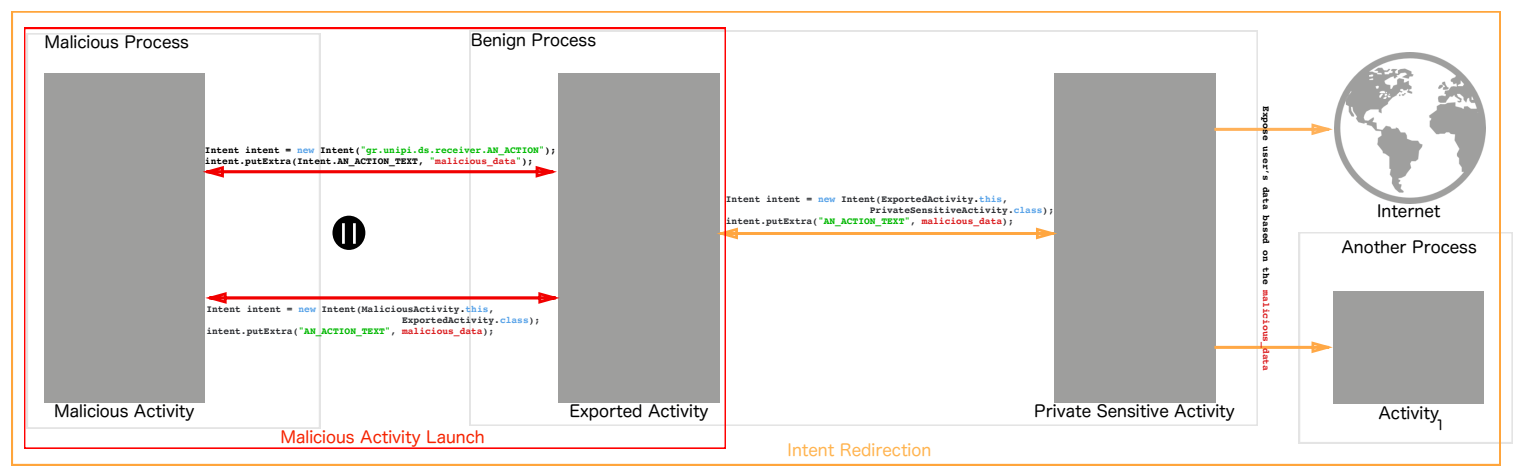

Figure 6.2 Intent Related Threats in Android Platform.

(activities that belong to different applications signed by the same certificate). Figure 6.2 depicts an example of such a case (refer to the inner part) in which a malicious application invokes directly a benign's exported activity.

At this point it should be stressed that a malicious application may launch another type of attack, called intent hijacking attack, by exporting an activity with the same intent-filter as the benign targeted application, thus trying to trick the user to select it for handling a specific intent request. This is because, whenever an application exports activities with the same intent-filter as other applications, the user is prompted to choose the application he prefers for managing the request. Moreover, whenever a malicious application exports an activity with the same intent-filter as a legitimate one which, however, is not installed on the device, the malicious application will handle the intent message by default, without the user being notified.

\subsubsection{Intent Redirection}

A variation of the malicious activity launch attack, described above, arises whenever a benign application that exports components, receives intents from untrusted or unknown sources and forwards them to other sensitive components. The exploitation of this vulnerability is known as intent redirection attack $[85,37,88]$ or equivalently next-intent vulnerability that generally falls under the confused deputy problem [91]. In such cases, a legitimate application may support a malicious application by forwarding malicious requests to other private components (depicted in the outer part of Figure 6.2).

In other words, a malicious application may launch a legitimate application's private component (via a publicly exported activity that interacts with the private one) with poisoned arguments or arbitrarily launch a legitimate application's component leading to private data 
leakages. So similarly to the malicious launch attack (see Section 6.1.1), a malevolent application instead of directly invoking another's application sensitive protected activity it can implicitly trigger the execution of a publicly exported application component that communicates and exchange data with the sensitive one, and force the intermediate component to perform a malicious act in it's behalf.

\subsection{Enhancement Mechanisms for Android Inter-Process Communication}

Chin et al.[31] are the first work focusing on flaws against Android Inter-Application communication with emphasis on intents manipulation, demonstrating activity and service hijacking attacks against legitimate applications. In the same direction, Wang et al. [88] and Tang et al. [85] disclose another class of vulnerability called intent redirection that expose legitimate application's components to data originated from untrusted sources that might cause data leakages. To protect applications and services against these threats various solutions have been proposed in literature varying from (a) static and dynamic analysis detection to (b) run time protection mechanisms.

\subsubsection{Static \& Dynamic Analysis Detection}

CHEX [63] introduces a static analysis tool, the first of its kind, that automatically tests Android applications for component hijacking vulnerabilities. Indeed, CHEX analyzes Android applications and detects possible flaws by conducting low-overhead reachability tests based on customized system dependency graphs. In the same direction, DroidChecker [30] relies on static analysis, however, it builds upon inter-procedural control flow graph and taint analysis.

Complementing such approaches, [70] proposes an Inter-Application Data Flow (IADF) analyzer leveraging on reverse engineering to extract and correlate the under examination application's intents, activities, and manifest features to detect potential leakages that might be caused due to Inter-application communication flaws. Similarly, El-Zawawy et al. [37] introduce another static analysis tool for detecting intent redirection vulnerabilities by investigating application's execution sequences.

Instead of relying upon static analysis, IntentFuzzer [96] deploys a dynamic intent fuzzing mechanism, requiring system-level modifications, in order to discover vulnerable applications that can be forced to act on behalf of a malicious application. In a slightly different approach LetterBomb [43] combines static analysis and symbolic execution to automatically generate exploits against Android applications and thus disclose vulnerable Inter-Component 
Communication (ICC) implementations, whereas Tang et al. [85] uses static and dynamic execution approaches to achieve the same goal.

\subsubsection{Runtime Protection}

IPC Inspection [42] protects applications against permission re-delegation attacks by introducing a system level runtime mechanism that reduces the privileges of the recipient application to the intersection of the recipient's and requester's permissions. The permission re-delegation attacks concern cases where an application with granted permissions perform privileged tasks on behalf of another application without permission.

Wang et al. [88] propose an open-source system-level mechanism, embedded in Android's Intent message mechanism, namely Morbs ${ }^{2}$, that can restrict applications' communication according to white-list policies introduced during applications' development. On the contrary, IEM [95] introduced an adaptable policy enforcement mechanism through an intent firewall service that was implemented in the Android framework.

In another scheme, Kaladharan et al. [50] propose a system-level encryption mechanism that protects Android's IPC against malicious interceptions. The proposed mechanism builds upon Android's binder inter-process communication mechanism and operates at kernel level, providing confidentiality services to Android applications. However, such an approach does not protect applications against hijacking attacks nor Intent redirection and malicious activity launch attacks.

Lyvas et al. [68] introduced a system-level mechanism that provides (a) intents source and/or destination authentication, and (b) confidentiality services for secure IPC between components. Moreover, the proposed solution named IntentAuth allows users' to define their policies, regarding which components are allowed to communicated with, in order to gain the control of their data.

Android OS does not support the use of policies for controlling the interactions of an application with others i.e., who is allowed to communicate with whom through implicit intents. In this context, the proposed approach supports this functionality, allowing users to define policies according to their needs.

To do so, a user interface was implemented (as Figure 6.3 depicts) through which a user can specify which application can receive intents from others, by extending Android's framework settings application. The apps that are allowed to communicate can be changed at any time by the user, and thus they are considered dynamic policies. At this point it should be stressed that IntentAuth and Android built-in settings application share the same UID as

\footnotetext{
2 https://github.com/mobile-security/Morbs
} 
parts of the Android system service $(U I D=1000)$. Therefore, IntentAuth can obtain user's preferences via settings application at runtime.

By default, IntentAuth policies allow any user-installed application to receive implicit intents containing data only from system/vendor applications or themselves while denying any interaction with implicit intents from other user-installed applications, following the least privilege principle. Via settings application, the end users are responsible for enabling or disabling an application's ability to receive intents from another application installed by the user.

The users' policies enable users to control the access to implicit intents requests, however, if this mechanism is not supported by secure communication between the components it can be easily circumvent. In order to ensure secure communication between the components, during the very first execution of a user-space application (invocation of startActivity (...) method belonging to Activity class), the IntentAuth service generates a public/private key pair $\left(\right.$ Keychain $\left._{n}\left(P U_{n}, P R_{n}\right)\right)$ and a symmetric key (SecretKey $)$ that will be used for the authentication of components and for data encryption/decryption purposes respectively.

Note that the service can identify whether the appropriate keys have been deployed by checking the existence of them in Android's Keystore, where they are securely stored. Moreover, due to SELinux policy enforcements in the Android Keystore, each application can generate and retrieve keys based on its UID only, and consequently an application cannot retrieve keys from another application if it does not share the same UID or a dedicated policy that allows such an action.

In this context, whenever a user-space application attempts to start an activity that requires the communication with other component(s), through an implicit intent, the IntentAuth service, which acts on behalf of the application, retrieves the keys of the corresponding (source) application from the keystore and uses them to sign (with the private key $\left(P R_{\text {source }}\right)$ ) and encrypt intent's data (with the symmetric key $\left(\right.$ Secret $_{\text {Key }}$ source $\left._{\text {) }}\right)$, thus enabling the verification (authentication) of the source activity and the protection of the data confidentiality. In fact,

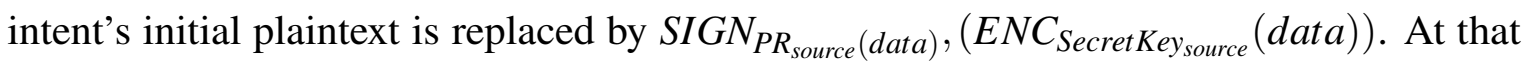
point, the source application is still unaware where the intent is going to be delivered because of its implicit nature. This is the responsibility of the activity task manager which specifies the appropriate activity (destination) for delivering the intent.

As soon as the activity task manager selects the destination activity, the IntentAuth consults the user defined policies to check if the communication between the source and the destination applications is allowed or not. If the communication is not allowed the execution is terminated. Otherwise IntentAuth validates the origin of the source application, by verifying the signature 
embedded to the intent's data, and proceeds with the decryption of the intent data, using the source application's symmetric key.

Afterwards, the IntentAuth mechanism prior the delivery of the intent to the destination component, it signs and encrypts it (i.e., $S I G N_{P R_{\text {dest }}}($ data $),\left(E N C_{\text {Secret Key }_{\text {dest }}}(\right.$ data $\left.\left.)\right)\right)$, in a way similar to the one followed by the source application. Then, the intent is forwarded to the target activity which, upon receipt, verifies the authenticity of the intent. If the verification is successful, it decrypts the intent data $D E C_{\text {Secret Key }_{\text {dest }}}\left(E N C_{\text {SecretKey dest }}(\right.$ data $\left.\left.)\right)\right)$. Figure 6.4 overviews this procedure. In this way, IntentAuth can achieve a secure delivery from one application to another considering the operating system as a trusted entity.

Moreover, IntentAuth was evaluated in terms of the execution overhead imposed to users' applications. IntentAuth was deployed on an emulator configured with Android framework version 11 using a proof of concept testbed with applications that communicate over IPC through implicit intents. The results indicate that the applications' execution time increases by $190 \mathrm{~ms}$, at the most, when IntentAuth is enabled. The execution overhead mentioned above from the enforcement of IntentAuth regards an application to application transmitted intent that contained a 26-byte string data. Of course, the introduced overhead is also affected by the size of data that should be encrypted for secure communication between the processes.

Overall, IntentAuth effectively safeguards the integrity, confidentiality, and authenticity of the Inter-Process communication in Android and is implemented as a system service that third party applications can employ for achieving secure communication with other Android components.

IntentAuth key management operations are deployed on top of Android's keystore system that by design is considered as a TEE. Moreover, according to the provisions of the SELinux policy of the Android keystore, applications are not allowed to access keys belonging to other applications; meaning that applications can manage keys belonging only to the same UID.

However, due to its intrinsic characteristics, it requires access to application keys. Thus, an additional SELinux policy was deployed for Android Keychain that allows system service $(U I D=1000)$ to gain access to user-space applications $(10000 \leq U I D \leq 19999)^{3}$ keys. Therefore, IntentAuth was developed as an operating system service, and hence it can be considered a trusted component similar to other OS components.

Indeed, with the support of such a mechanism, IntentAuth gains access only to the key chains and symmetric keys of the involved user-space applications without exposing them to other user-space applications. As such, IntentAuth safeguards the integrity, confidentiality, and authenticity of the keys required for the secure communication of the intents.

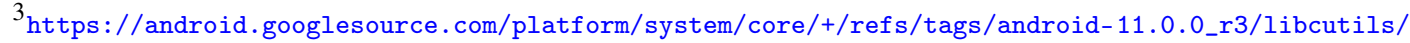
include/private/android_filesystem_config.h
} 


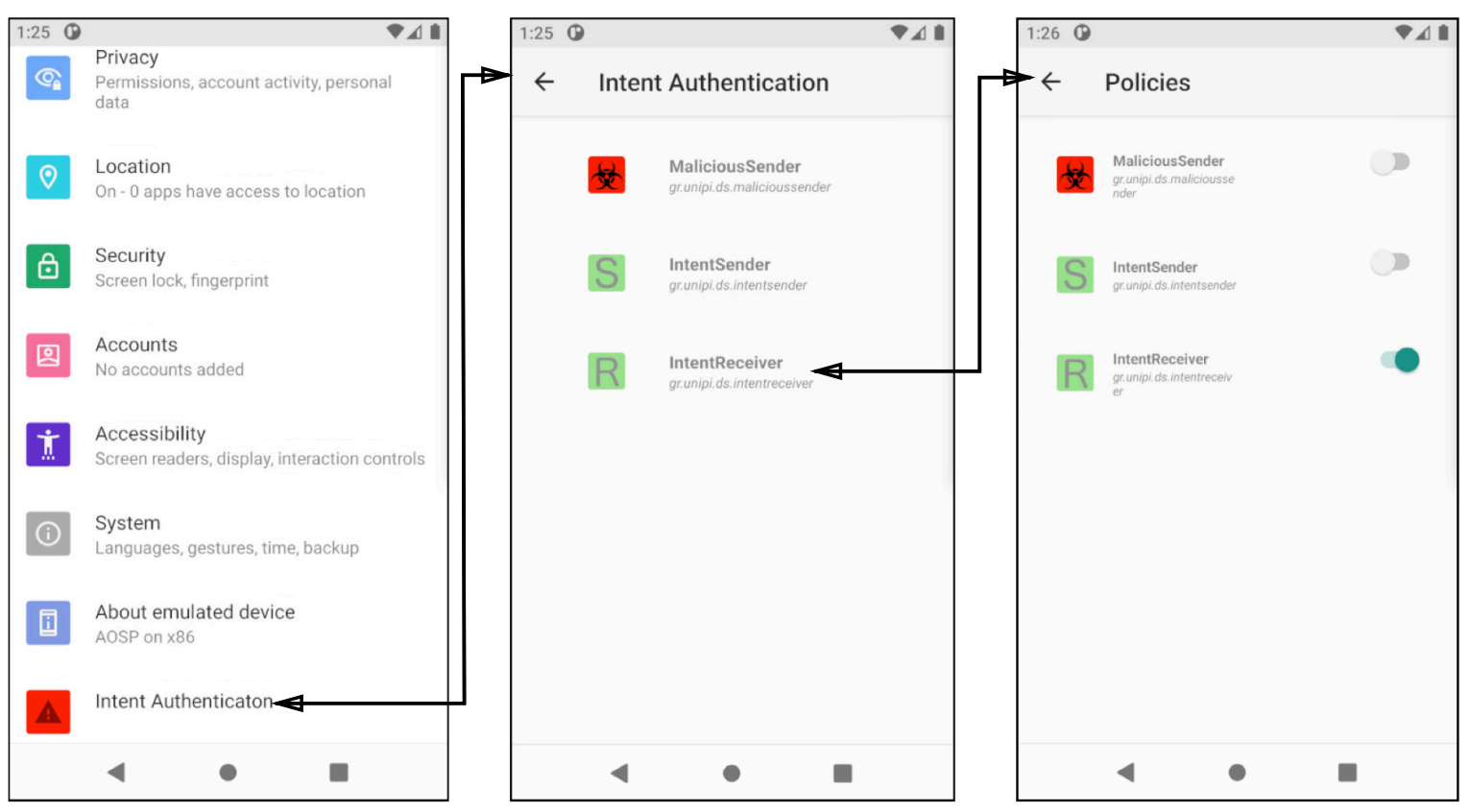

Figure 6.3 IntentAuth User-Defined Policies via Android (Version 11) Settings Application.

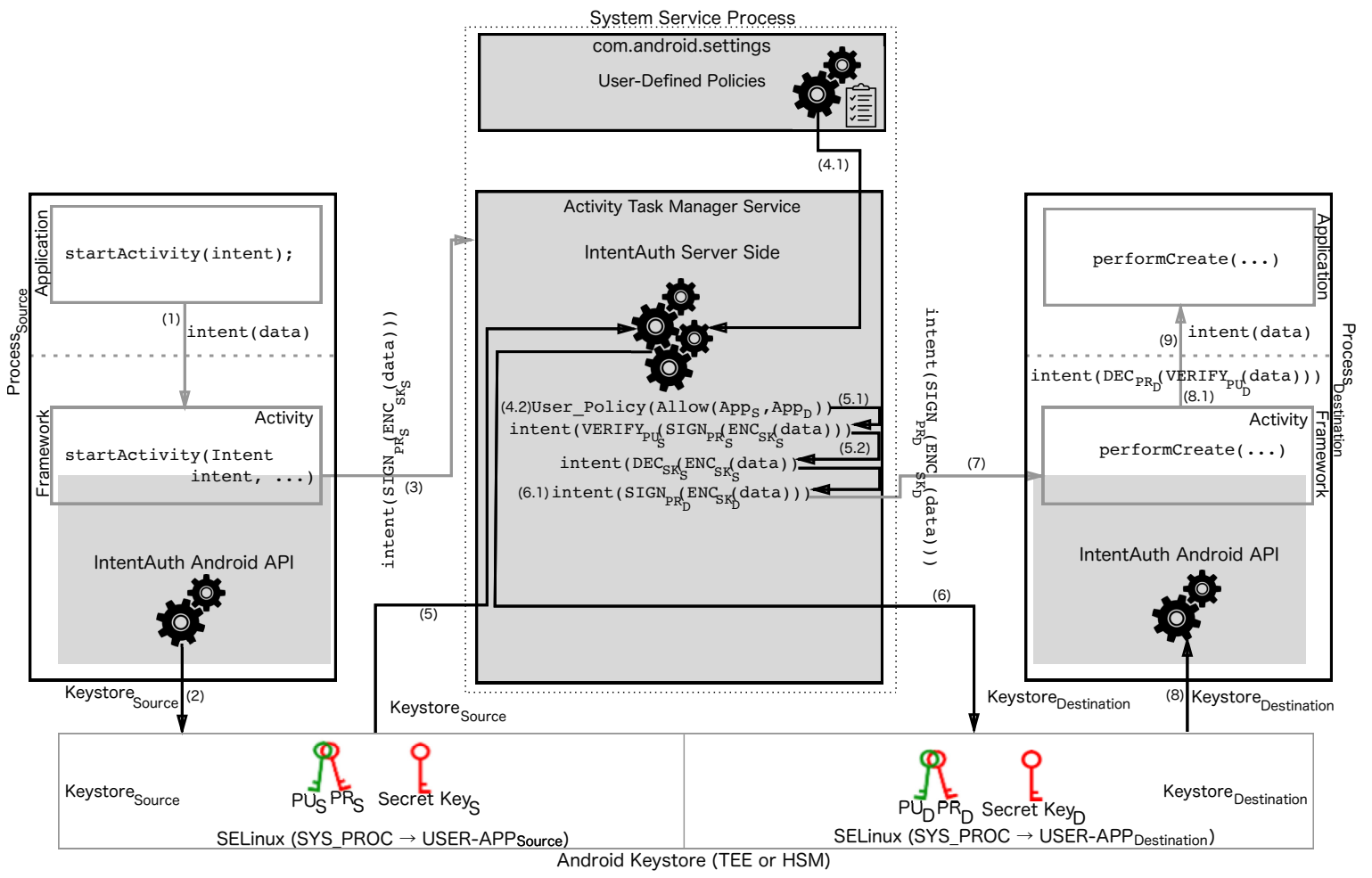

Figure 6.4 IntentAuth High-Level Overview. 



\section{Chapter 7}

\section{Conclusions}

Android is one of the dominant mobile operating systems with millions of applications available for its plethora of users. Several system and application-level mechanisms protect the security of the devices and the privacy of the users. However, there are several threats against user's privacy and security due to the platform's peculiarities. This dissertation copes with the permission map compilation and the threats of task, activity, intent hijacking, malicious activity launch, and the intent redirection. As such threats are eminent security flaws requiring high attention, several mechanisms have been introduced to identify deficiencies, discover vulnerabilities, and prevent exploitation in this thesis.

For the Android permission map generation, the Dypermin tool was introduced based on a method capable of compiling the permission map for any given Android version without generating any false positives. In addition, Dypermin is transparent to the underlying operating system, meaning that there is no need for any operating system modifications.

Additionally, Anactijax was deployed to identify vulnerable applications and configurations regarding task and activity hijacking threats. Moreover, the system level prevention mechanism TaskAuth was introduced that controls activities interactions based on predefined dynamic policies. TaskAuth demands system-level modifications, but it is transparent both to end-users and developers.

Finally, regarding the threats of intent hijacking, malicious activity launch, and intent redirection, a system-based security service named IntentAuth was introduced to enable encrypted IPC between applications and provide users the capacity to control applications' IPC through adaptable policies. Although IntentAuth requires system-level modifications, it is completely transparent to applications and users and does not impose any significant overhead on users' experience navigation.

We are currently focusing on prioritizing the Android framework threats, aiming to extend our work to protect all forms of the Inter-Process Communication scheme of the Android 
platform with the redesign of the presented in this thesis prevention mechanism of IntentAuth without the need for underlying operating system modification able to provide a combined trust model with dynamic policies given by both developers and end-users. 


\section{Bibliography}

[1] Android Open Source Project. Application Fundamentals. Technical report, 2019. URL https://developer .android.com/guide/components/fundamentals. Cited March 22nd 2020.

[2] Android Open Source Project. Intents and Intent Filters. Technical report, 2019. URL https://developer.android.com/guide/components/intents-filters. Cited March 22nd 2020.

[3] Android Open Source Project. Processes and Threads Overview. Technical report, 2019. URL https://developer.android.com/guide/components/ processes-and-threads. Cited March 25th 2020.

[4] Android Open Source Project. Understand Tasks and Back Stack. Technical report, 2019. URL https://developer.android.com/guide/components/activities/ tasks-and-back-stack. Cited March 22nd 2020.

[5] Android Open Source Project. Implementing SELinux. Technical report, 2020. URL https://source.android.com/security/selinux/implement. Cited September 23rd 2020.

[6] Android Open Source Project. Security Enhancements in Android 1.5 through 4.1. Technical report, 2020. URL https://source.android.com/security/enhancements/ enhancements41. Cited September 23rd 2020.

[7] Android Open Source Project. Security and Privacy Enhancements in Android 10. Technical report, 2020. URL https://source.android.com/security/enhancements/ enhancements10. Cited September 23rd 2020.

[8] Android Open Source Project. Security Enhancements in Android 4.2. Technical report, 2020. URL https://source.android.com/security/enhancements/enhancements 42. Cited September 23rd 2020.

[9] Android Open Source Project. Security Enhancements in Android 4.3. Technical report, 2020. URL https://source.android.com/security/enhancements/enhancements 43. Cited September 23rd 2020.

[10] Android Open Source Project. Security Enhancements in Android 4.4. Technical report, 2020. URL https://source.android.com/security/enhancements/enhancements 44. Cited September 23rd 2020. 
[11] Android Open Source Project. Security Enhancements in Android 5.0. Technical report, 2020. URL https://source.android.com/security/enhancements/enhancements50. Cited September 23rd 2020.

[12] Android Open Source Project. Security Enhancements in Android 6.0. Technical report, 2020. URL https://source.android.com/security/enhancements/enhancements60. Cited September 23rd 2020.

[13] Android Open Source Project. Security Enhancements in Android 7.0. Technical report, 2020. URL https://source.android.com/security/enhancements/enhancements70. Cited September 23rd 2020.

[14] Android Open Source Project. Security Enhancements in Android 8.0. Technical report, 2020. URL https://source.android.com/security/enhancements/enhancements 80 . Cited September 23rd 2020.

[15] Android Open Source Project. Security Enhancements in Android 9.0. Technical report, 2020. URL https://source . android.com/security/enhancements/enhancements9. Cited September 23rd 2020.

[16] Android Open Source Project. Android Architecture. Technical report, 2020. URL https://source.android.com/devices/architecture. Cited March 24th 2020.

[17] Android Open Source Project. Gatekeeper. Technical report, 2020. URL https:// source.android.com/security/authentication/gatekeeper. Cited October 10th 2020.

[18] Android Open Source Project. Android Gradle plugin release notes. Technical report, 2020. URL https://developer.android.com/studio/releases/gradle-plugin. Cited October 29th 2020.

[19] Android Open Source Project. Android Keystore System. Technical report, 2020. URL https://developer.android.com/training/articles/keystore. Cited October 30th 2020.

[20] Android Open Source Project. Permissions on Android. Technical report, 2020. URL https://developer. android.com/guide/topics/permissions/overview. Cited September 23 rd 2020.

[21] Android Open Source Project. Application Signing. Technical report, 2020. URL https://source. android.com/security/apksigning. Cited Octomber 20th 2020.

[22] Android Open Source Project. Trusty TEE. Technical report, 2020. URL https: //source.android.com/security/trusty. Cited September 23rd 2020.

[23] Daniel Arp, Michael Spreitzenbarth, Malte Hubner, Hugo Gascon, and Konrad Rieck. Drebin: Effective and explainable detection of android malware in your pocket. In 21st Annual Network and Distributed System Security Symposium, NDSS 2014, San Diego, California, USA, February 23-26, 2013, 2014.

[24] Kathy Wain Yee Au, Yi Fan Zhou, Zhen Huang, and David Lie. Pscout: analyzing the android permission specification. In Proceedings of the 2012 ACM conference on Computer and communications security, CCS '12, pages 217-228, New York, NY, USA, 2012. ACM. ISBN 978-1-4503-1651-4. 
[25] Michael Backes, Sven Bugiel, Erik Derr, Patrick McDaniel, Damien Octeau, and Sebastian Weisgerber. On demystifying the android application framework: Re-visiting android permission specification analysis. In 25th USENIX Security Symposium (USENIX Security 16), pages 1101-1118, Austin, TX, 2016. USENIX Association. ISBN 978-1-931971-32-4.

[26] Alexandre Bartel, Jacques Klein, Martin Monperrus, and Yves Le Traon. Static analysis for extracting permission checks of a large scale framework: The challenges and solutions for analyzing android. IEEE Transactions on Software Engineering (TSE), 2014.

[27] Parnika Bhat and Kamlesh Dutta. A survey on various threats and current state of security in android platform. ACM Computing Surveys (CSUR), 52(1):1-35, 2019.

[28] Andrew D. Birrell and Bruce Jay Nelson. Implementing Remote Procedure Calls. ACM Trans. Comput. Syst., 2(1):39-59, February 1984. ISSN 0734-2071. doi: 10. 1145/2080.357392. URL https://doi.org/10.1145/2080.357392.

[29] Denis Bogdanas. Dperm: Assisting the migration of android apps to runtime permissions. CoRR, abs/1706.05042, 2017. URL http://arxiv.org/abs/1706.05042.

[30] Patrick PF Chan, Lucas CK Hui, and Siu-Ming Yiu. Droidchecker: analyzing android applications for capability leak. In Proceedings of the fifth ACM conference on Security and Privacy in Wireless and Mobile Networks, pages 125-136, 2012.

[31] Erika Chin, Adrienne Porter Felt, Kate Greenwood, and David Wagner. Analyzing inter-application communication in android. In Proceedings of the 9th international conference on Mobile systems, applications, and services, pages 239-252, 2011.

[32] Lucas Davi, Alexandra Dmitrienko, Ahmad-Reza Sadeghi, and Marcel Winandy. Privilege escalation attacks on android. In international conference on Information security, pages 346-360. Springer, 2010.

[33] Jeffrey Dean, David Grove, and Craig Chambers. Optimization of object-oriented programs using static class hierarchy analysis. In ECOOP '95 Proceedings of the 9th European Conference on Object-Oriented Programming, ECOOP '95, pages 77-101, London, UK, 1995. Springer-Verlag. ISBN 3-540-60160-0.

[34] XDA Developers. Xposed framework hub, 2020. URL https://www.xda-developers. com/xposed-framework-hub/.

[35] Vasiliki Diamantopoulou, Christos Kalloniatis, Christos Lyvas, Konstantinos Maliatsos, Matthieu Gay, Athanasios Kanatas, and Costas Lambrinoudakis. Aligning the concepts of risk, security and privacy towards the design of secure intelligent transport systems. In Computer Security, pages 170-184. Springer, 2020. doi: https://doi.org/10.1007/978-3-030-64330-0_11. URL https://link.springer.com/ chapter/10.1007/978-3-030-64330-0_11.

[36] Yu Ding, Zhuo Peng, Yuanyuan Zhou, and Chao Zhang. Android low entropy demystified. In 2014 IEEE International Conference on Communications (ICC), pages 659-664. IEEE, 2014. 
[37] Mohamed A El-Zawawy, Eleonora Losiouk, and Mauro Conti. Do not let next-intent vulnerability be your next nightmare: type system-based approach to detect it in android apps. International Journal of Information Security, pages 1-20, 2020.

[38] William Enck, Machigar Ongtang, and Patrick McDaniel. Understanding android security. IEEE Security and Privacy, 7(1):50-57, January 2009. ISSN 1540-7993. doi: 10.1109/MSP.2009.26. URL http://dx.doi.org/10.1109/MSP.2009.26.

[39] William Enck, Peter Gilbert, Byung-Gon Chun, Landon P. Cox, Jaeyeon Jung, Patrick McDaniel, and Anmol N. Sheth. Taintdroid: An information flow tracking system for real-time privacy monitoring on smartphones. Commun. ACM, 57(3):99-106, March 2014. ISSN 0001-0782. doi: 10.1145/2494522. URL https://doi.org/10. $1145 / 2494522$.

[40] P. Faruki, A. Bharmal, V. Laxmi, V. Ganmoor, M. S. Gaur, M. Conti, and M. Rajarajan. Android security: A survey of issues, malware penetration, and defenses. IEEE Communications Surveys Tutorials, 17(2):998-1022, 2015.

[41] Adrienne Porter Felt, Erika Chin, Steve Hanna, Dawn Song, and David Wagner. Android permissions demystified. In Proceedings of the 18th ACM Conference on Computer and Communications Security, CCS '11, pages 627-638, New York, NY, USA, 2011. ACM. ISBN 978-1-4503-0948-6.

[42] Adrienne Porter Felt, Helen J Wang, Alexander Moshchuk, Steve Hanna, and Erika Chin. Permission re-delegation: Attacks and defenses. In USENIX security symposium, volume 30, page 88, 2011.

[43] Joshua Garcia, Mahmoud Hammad, Negar Ghorbani, and Sam Malek. Automatic generation of inter-component communication exploits for android applications. In Proceedings of the 2017 11th Joint Meeting on Foundations of Software Engineering, pages 661-671, 2017.

[44] Dimitris Geneiatakis, Igor Nai Fovino, Ioannis Kounelis, and Paquale Stirparo. A permission verification approach for android mobile applications. Computers \& Security, 49:192-205, 2015.

[45] Hector Marco Gisbert and Ismael Ripoll. On the effectiveness of nx, ssp, renewssp, and aslr against stack buffer overflows. In 2014 IEEE 13th International Symposium on Network Computing and Applications, pages 145-152. IEEE, 2014.

[46] Google LLC. Android Enterprise Security White Paper. Technical report, 2020. URL https://static.googleusercontent.com/media/www.android.com/en//static/2016/ pdfs/enterprise/Android_Enterprise_Security_White_Paper_2019.pdf. Cited March 22nd 2020.

[47] Norm Hardy. The confused deputy: (or why capabilities might have been invented). ACM SIGOPS Operating Systems Review, 22(4):36-38, 1988.

[48] Sungjae Hwang, Sungho Lee, and Sukyoung Ryu. All about activity injection: Threats, semantics, detection, and defense. Software: Practice and Experience, 50(7):10611086, 2020. doi: 10.1002/spe.2792. URL https://onlinelibrary.wiley.com/doi/abs/ $10.1002 /$ spe. 2792 . 
[49] Ajay Kumar Jha, Sunghee Lee, and Woo Jin Lee. Developer mistakes in writing android manifests: An empirical study of configuration errors. In 2017 IEEE/ACM 14th International Conference on Mining Software Repositories (MSR), pages 25-36, 2017. doi: 10.1109/MSR.2017.41.

[50] Yadu Kaladharan, Prabhaker Mateti, and KP Jevitha. An encryption technique to thwart android binder exploits. In Intelligent Systems Technologies and Applications, pages 13-21. Springer, 2016.

[51] Christos Kalloniatis, Vasiliki Diamantopoulou, Konstantinos Kotis, Christos Lyvas, Konstantinos Maliatsos, Matthieu Gay, Athanasios G Kanatas, and Costas Lambrinoudakis. Towards the design of an assurance framework for increasing security and privacy in connected vehicles. International Journal of Internet of Things and Cyber-Assurance, 1(3-4):244-266, 2020. ISSN 2059-7967. doi: https://doi.org/10. 1504/IJITCA.2020.112574. URL https://www.inderscienceonline.com/doi/abs/10. 1504/IJITCA. 2020.112574.

[52] Anatoli Kalysch, Mark Deutel, and Tilo Müller. Template-based android inter process communication fuzzing. In Proceedings of the 15th International Conference on Availability, Reliability and Security, pages 1-6, 2020.

[53] Foutse Khomh, Hao Yuan, and Ying Zou. Adapting Linux for mobile platforms: An empirical study of Android. In 2012 28th IEEE international conference on software maintenance (ICSM), pages 629-632. IEEE, 2012.

[54] Vasileios Kouliaridis, Georgios Kambourakis, Dimitris Geneiatakis, and Nektaria Potha. Two anatomists are better than one-dual-level android malware detection. Symmetry, 12(7), 2020. ISSN 2073-8994. doi: 10.3390/sym12071128. URL https: //www.mdpi.com/2073-8994/12/7/1128.

[55] Sungho Lee, Sungjae Hwang, and Sukyoung Ryu. All about activity injection: threats, semantics, and detection. In 2017 32nd IEEE/ACM International Conference on Automated Software Engineering (ASE), pages 252-262. IEEE, 2017.

[56] Ondřej Lhoták and Laurie Hendren. Scaling java points-to analysis using spark. In Proceedings of the 12th international conference on Compiler construction, CC'03, pages 153-169, New York, NY, USA, 2003. ACM. ISBN 3-540-00904-3.

[57] Jin Li, Lichao Sun, Qiben Yan, Zhiqiang Li, Witawas Srisa-An, and Heng Ye. Significant permission identification for machine-learning-based android malware detection. IEEE Transactions on Industrial Informatics, 14(7):3216-3225, 2018.

[58] Li Li, Tegawendé F Bissyandé, Yves Le Traon, and Jacques Klein. Accessing inaccessible android apis: An empirical study. In The 32nd International Conference on Software Maintenance and Evolution (ICSME 2016), 2016.

[59] Li Li, Tegawendé F Bissyandé, Damien Octeau, and Jacques Klein. Droidra: taming reflection to support whole-program analysis of android apps. In Proceedings of the 25th International Symposium on Software Testing and Analysis, ISSTA 2016, pages 318-329, New York, NY, USA, 2016. ACM. ISBN 978-1-4503-4390-9. 
[60] Steffen Liebergeld and Matthias Lange. Android security, pitfalls and lessons learned. In Information Sciences and Systems 2013, pages 409-417. Springer, 2013.

[61] Jie Liu, Diyu Wu, and Jingling Xue. Tdroid: exposing app switching attacks in android with control flow specialization. In Proceedings of the 33rd ACM/IEEE International Conference on Automated Software Engineering, pages 236-247, 2018.

[62] Kangjie Lu, Chengyu Song, Byoungyoung Lee, Simon P. Chung, Taesoo Kim, and Wenke Lee. Aslr-guard: Stopping address space leakage for code reuse attacks. In Proceedings of the 22nd ACM SIGSAC Conference on Computer and Communications Security, CCS '15, page 280-291, New York, NY, USA, 2015. Association for Computing Machinery. ISBN 9781450338325. doi: 10.1145/2810103.2813694. URL https://doi.org/10.1145/2810103.2813694.

[63] Long Lu, Zhichun Li, Zhenyu Wu, Wenke Lee, and Guofei Jiang. Chex: statically vetting android apps for component hijacking vulnerabilities. In Proceedings of the 2012 ACM conference on Computer and communications security, pages 229-240, 2012.

[64] Christos Lyvas, Nikolaos Pitropakis, and Costas Lambrinoudakis. The far side of mobile application integrated development environments. In International Conference on Trust and Privacy in Digital Business, pages 111-122. Springer, 2016. doi: https: //doi.org/10.1007/978-3-319-44341-6_8. URL https://link.springer.com/chapter/ 10.1007/978-3-319-44341-6_8.

[65] Christos Lyvas, Costas Lambrinoudakis, and Dimitris Geneiatakis. Dypermin: Dynamic permission mining framework for android platform. Computers \& Security, 77: 472-487, 2018. ISSN 0167-4048. doi: https://doi.org/10.1016/j.cose.2018.05.007. URL https://www.sciencedirect.com/science/article/pii/S0167404818304954.

[66] Christos Lyvas, Costas Lambrinoudakis, and Dimitris Geneiatakis. On android's activity hijacking prevention. Computers \& Security, 111:102468, 2021. ISSN 0167-4048. doi: https://doi.org/10.1016/j.cose.2021.102468. URL https://www. sciencedirect. com/science/article/pii/S0167404821002923.

[67] Christos Lyvas, Christoforos Ntantogian, and Christos Xenakis. [m]allotropism: a metamorphic engine for malicious software variation development. International Journal of Information Security, pages 1-18, 2021. doi: https://doi.org/ 10.1007/s10207-021-00541-y. URL https://link.springer.com/article/10.1007/ s10207-021-00541-y.

[68] Christos Lyvas, Costas Lambrinoudakis, and Dimitris Geneiatakis. IntentAuth: Securing Android's Intent Based Inter-Process Communication. International Journal of Information Security, 2021 (Under Minor Revision).

[69] Konstantinos Maliatsos, Christos Lyvas, Panagiotis Pantazopoulos, Costas Lambrinoudakis, Athanasios Kanatas, Matthieu Gay, and Angelos Amditis. Standardizing security evaluation criteria for connected vehicles: A modular protection profile. In 2019 IEEE Conference on Standards for Communications and Networking (CSCN), pages 1-7. IEEE, 2019. doi: https://doi.org/10.1109/CSCN.2019.8931344. URL https://ieeexplore.ieee.org/document/8931344. 
[70] Hafiz Muhammad Arslan Maqsood, Kashif Naseer Qureshi, Faisal Bashir, and Najam Ul Islam. Privacy leakage through exploitation of vulnerable inter-app communication on android. In 2019 13th International Conference on Open Source Systems and Technologies (ICOSST), pages 1-6. IEEE, 2019.

[71] René Mayrhofer, Jeffrey Vander Stoep, Chad Brubaker, and Nick Kralevich. The Android Platform Security Model. ACM Trans. Priv. Secur., 24(3), April 2021. ISSN 2471-2566. doi: 10.1145/3448609. URL https://doi.org/10.1145/3448609.

[72] Niall McLaughlin, Jesus Martinez del Rincon, BooJoong Kang, Suleiman Yerima, Paul Miller, Sakir Sezer, Yeganeh Safaei, Erik Trickel, Ziming Zhao, Adam Doupé, and Gail Joon Ahn. Deep android malware detection. In Proceedings of the Seventh ACM on Conference on Data and Application Security and Privacy, CODASPY '17, page 301-308, New York, NY, USA, 2017. Association for Computing Machinery. ISBN 9781450345231. doi: 10.1145/3029806.3029823. URL https://doi.org/10. $1145 / 3029806.3029823$.

[73] Ana Milanova, Atanas Rountev, and Barbara G. Ryder. Parameterized object sensitivity for points-to and side-effect analyses for java. In Proceedings of the 2002 ACM SIGSOFT international symposium on Software testing and analysis, ISSTA '02, pages 1-11, New York, NY, USA, 2002. ACM. ISBN 1-58113-562-9.

[74] Jon Oberheide and Charlie Miller. Dissecting the android bouncer. SummerCon2012, New York, 95:110, 2012.

[75] Damien Octeau, Patrick McDaniel, Somesh Jha, Alexandre Bartel, Eric Bodden, Jacques Klein, and Yves Le Traon. Effective inter-component communication mapping in android: An essential step towards holistic security analysis. In Presented as part of the 22nd USENIX Security Symposium (USENIX Security 13), pages 543-558, 2013.

[76] Carlos Pacheco, Shuvendu K. Lahiri, Michael D. Ernst, and Thomas Ball. Feedbackdirected random test generation. In ICSE'07, Proceedings of the 29th International Conference on Software Engineering, pages 75-84, Minneapolis, MN, USA, May 2325, 2007.

[77] Naser Peiravian and Xingquan Zhu. Machine learning for android malware detection using permission and api calls. In 2013 IEEE 25th international conference on tools with artificial intelligence, pages 300-305. IEEE, 2013.

[78] Nikolaos Pitropakis, Christos Lyvas, and Costas Lambrinoudakis. The greater the power, the more dangerous the abuse: facing malicious insiders in the cloud. In International Conference on Cloud Computing, GRIDs, and Virtualization, Track Security and Privacy in Cloud Computing (SEPRICC 2017), pages 156-161. IARIA Xpert Publishing Services, 2017.

[79] Chuangang Ren, Yulong Zhang, Hui Xue, Tao Wei, and Peng Liu. Towards discovering and understanding task hijacking in android. In 24th USENIX Security Symposium (USENIX Security 15), pages 945-959, 2015.

[80] Chuangang Ren, Peng Liu, and Sencun Zhu. Windowguard: Systematic protection of gui security in android. In 2017 Network and Distributed System Security (NDSS) Symposium, 2017. 
[81] Yunlong Ren, Yue Li, Fangfang Yuan, and Fangjiao Zhang. Hijacking activity technology analysis and research in android system. In International Conference on Trustworthy Computing and Services, pages 46-53. Springer, 2013.

[82] Stephen Smalley and Robert Craig. Security Enhanced (SE) Android: Bringing Flexible MAC to Android. In NDSS, volume 310, pages 20-38, 2013.

[83] Patrícia Gomes Soares. On remote procedure call. In Proceedings of the 1992 Conference of the Centre for Advanced Studies on Collaborative Research - Volume 2, CASCON '92, page 215-267. IBM Press, 1992.

[84] Geran Tam and Aaron Hunter. Machine learning to identify android malware. In 2018 9th IEEE Annual Ubiquitous Computing, Electronics \& Mobile Communication Conference (UEMCON), pages 1-5. IEEE, 2018.

[85] Junjie Tang, Xingmin Cui, Ziming Zhao, Shanqing Guo, Xinshun Xu, Chengyu Hu, Tao Ban, and Bing Mao. Nivanalyzer: a tool for automatically detecting and verifying next-intent vulnerabilities in android apps. In 2017 IEEE International Conference on Software Testing, Verification and Validation (ICST), pages 492-499. IEEE, 2017.

[86] Ximing Tang, Tao Song, Kun Wang, and Alei Liang. Fine-Grained Access Control on Android Through Behavior Monitoring. In Advances in Computer Communication and Computational Sciences, pages 525-532. Springer, 2019.

[87] Raja Vallée-Rai, Phong Co, Etienne Gagnon, Laurie Hendren, Patrick Lam, and Vijay Sundaresan. Soot - a java bytecode optimization framework. In CASCON 99, Proceedings of the 1999 conference of the Centre for Advanced Studies on Collaborative research. IBM Press, 1999.

[88] Rui Wang, Luyi Xing, XiaoFeng Wang, and Shuo Chen. Unauthorized origin crossing on mobile platforms: Threats and mitigation. In Proceedings of the 2013 ACM SIGSAC conference on Computer \& communications security, pages 635-646, 2013.

[89] Zhaoguo Wang, Chenglong Li, Yi Guan, Yibo Xue, and Yingfei Dong. Activityhijacker: Hijacking the android activity component for sensitive data. In 201625 th International Conference on Computer Communication and Networks (ICCCN), pages 1-9. IEEE, 2016.

[90] Xuetao Wei, Lorenzo Gomez, Iulian Neamtiu, and Michalis Faloutsos. Permission evolution in the android ecosystem. In Proceedings of the 28th Annual Computer Security Applications Conference, pages 31-40, 2012.

[91] Jianliang Wu, Tingting Cui, Tao Ban, Shanqing Guo, and Lizhen Cui. Paddyfrog: systematically detecting confused deputy vulnerability in android applications. Security and Communication Networks, 8(13):2338-2349, 2015.

[92] Min-Hao Wu, Fu-Hau Hsu, Ting-Cheng Chang, and Liang Hsuan Lin. Preventing activity hijacking attacks in android app. In 2019 IEEE Eurasia Conference on Biomedical Engineering, Healthcare and Sustainability (ECBIOS), pages 170-173. IEEE, 2019. 
[93] Yinhao Xiao, Guangdong Bai, Jian Mao, Zhenkai Liang, and Wei Cheng. Privilege leakage and information stealing through the android task mechanism. In 2017 IEEE Symposium on Privacy-Aware Computing (PAC), pages 152-163. IEEE, 2017.

[94] Luyi Xing, Xiaorui Pan, Rui Wang, Kan Yuan, and XiaoFeng Wang. Upgrading your android, elevating my malware: Privilege escalation through mobile os updating. In Proceedings of the 2014 IEEE Symposium on Security and Privacy, SP '14, pages 393408, Washington, DC, USA, 2014. IEEE Computer Society. ISBN 978-1-4799-4686-0. doi: 10.1109/SP.2014.32. URL http://dx.doi.org/10.1109/SP.2014.32.

[95] Carter Yagemann and Wenliang Du. Intentio ex machina: Android intent access control via an extensible application hook. In European Symposium on Research in Computer Security, pages 383-400. Springer, 2016.

[96] Kun Yang, Jianwei Zhuge, Yongke Wang, Lujue Zhou, and Haixin Duan. Intentfuzzer: detecting capability leaks of android applications. In Proceedings of the 9th ACM symposium on Information, computer and communications security, pages 531-536, 2014.

[97] Ayman Youssef and Ahmed F. Shosha. Quantitave dynamic taint analysis of privacy leakage in android arabic apps. In Proceedings of the 12th International Conference on Availability, Reliability and Security, ARES '17, New York, NY, USA, 2017. Association for Computing Machinery. ISBN 9781450352574. doi: 10.1145/3098954. 3105827. URL https://doi.org/10.1145/3098954.3105827.

[98] Jianing Zhang, Xingtao Zhuang, and Yunfang Chen. Android malware detection combined with static and dynamic analysis. In Proceedings of the 2019 the 9th International Conference on Communication and Network Security, ICCNS 2019, page 6-10, New York, NY, USA, 2019. Association for Computing Machinery. ISBN 9781450376624. doi: 10.1145/3371676.3371685. URL https://doi.org/10.1145/ 3371676.3371685 .

[99] Yuan Zhang, Min Yang, Bingquan Xu, Zhemin Yang, Guofei Gu, Peng Ning, X. Sean Wang, and Binyu Zang. Vetting undesirable behaviors in android apps with permission use analysis. In Proceedings of the 2013 ACM SIGSAC Conference on Computer \&\#38; Communications Security, CCS '13, pages 611-622, New York, NY, USA, 2013. ACM. ISBN 978-1-4503-2477-9. doi: 10.1145/2508859.2516689.

[100] Yury Zhauniarovich and Olga Gadyatskaya. Small Changes, Big Changes: An Updated View on the Android Permission System, pages 346-367. Springer International Publishing, Cham, 2016. ISBN 978-3-319-45719-2. doi: 10.1007/978-3-319-45719-2_16. URL https://doi.org/10.1007/978-3-319-45719-2_16. 



\section{Appendix A}

\section{Intents Usage Examples}

\section{A.1 Implicit Intents}

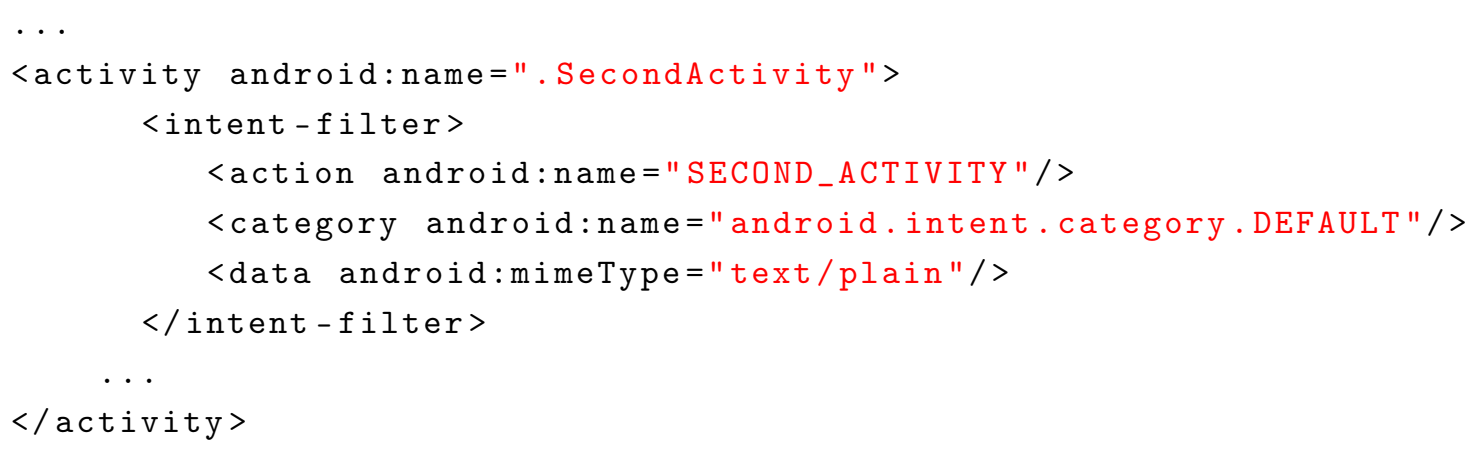

Listing A.1 Declaration of Activity Initiated by Implicit Intent.

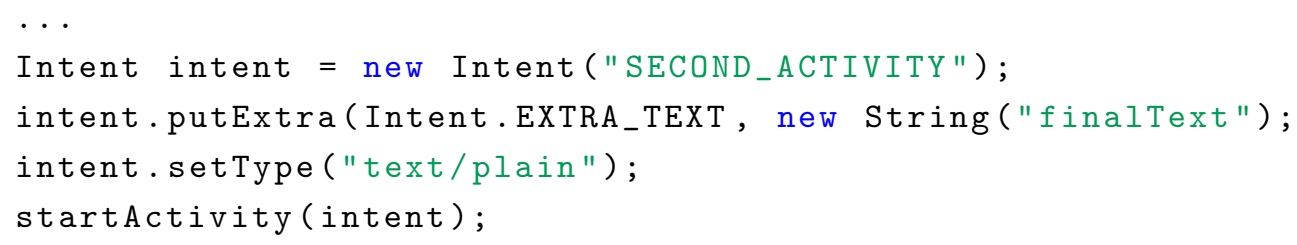

Listing A.2 Invocation of an Activity with an Implicit Intent.

\section{A.2 Explicit Intents}

. .

2 <activity android:name=". SecondActivity"> 
$4</$ activity $>$

5

Listing A.3 Declaration of Activity Initiated by Explicit Intent.

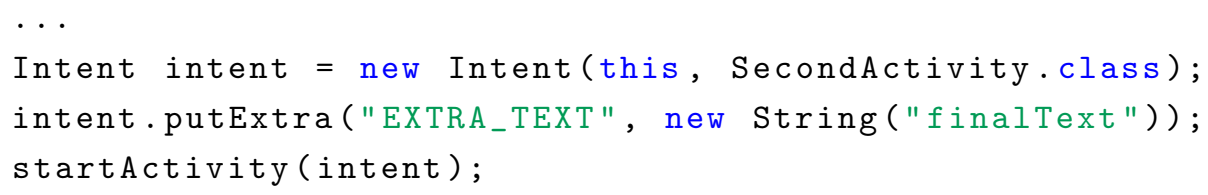

Listing A.4 Invocation of an Activity with an Explicit Intent. 


\section{Appendix B}

\section{Anactijax Generated Use Cases Examples}

\section{B.1 Activity Injection Against any Benign Application}

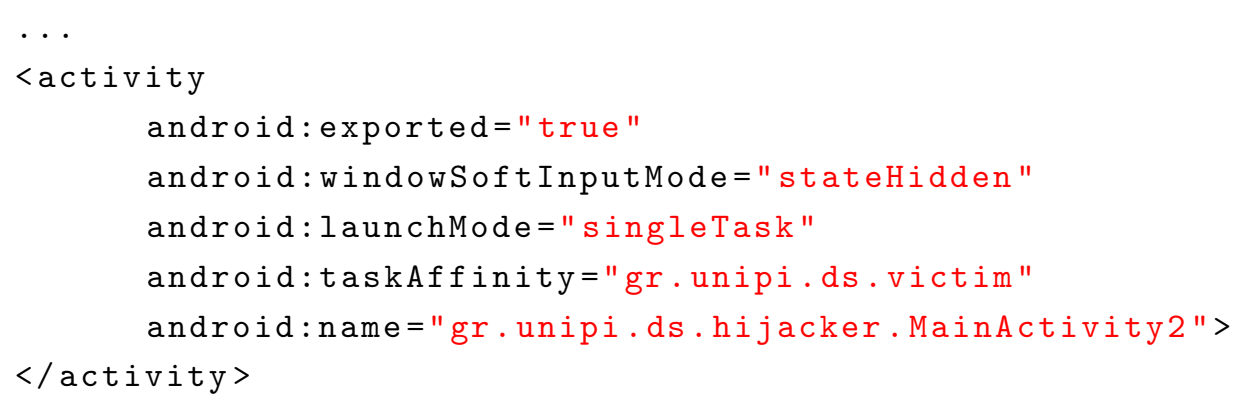

Listing B.1 Example of Manifest File Generated by Anactijax for Malicious Activity Injection Against a Default Benign Application.

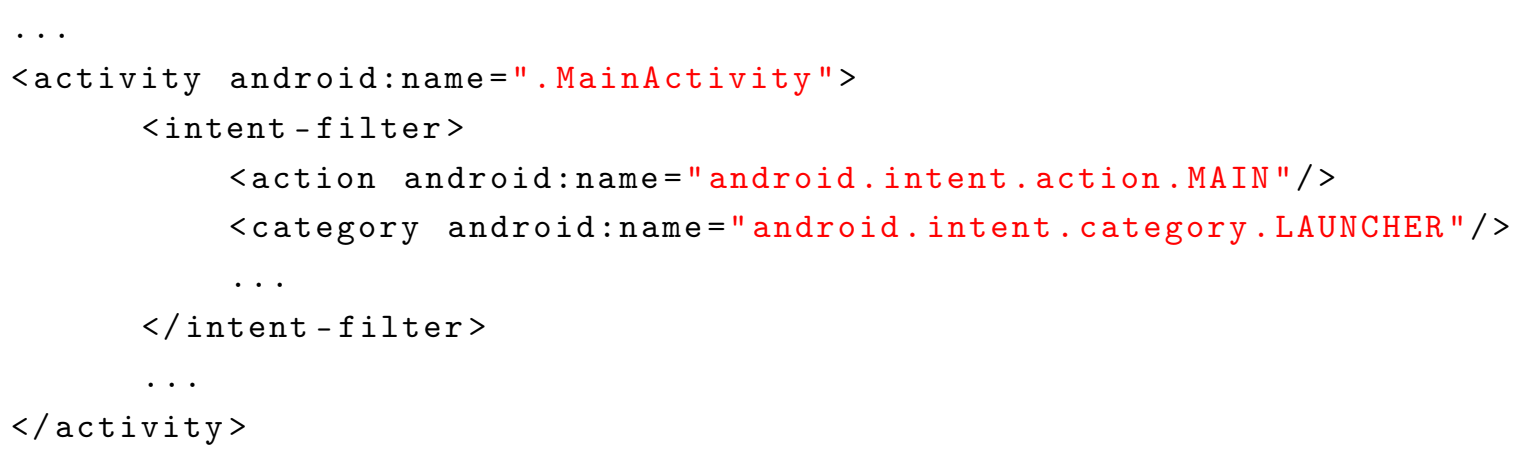

Listing B.2 Example of Manifest File Generated by Anactijax for Default Vulnerable Benign Application. 


\section{B.2 Activity Injection Against Vulnerable Benign Applica- tion's Tasks}

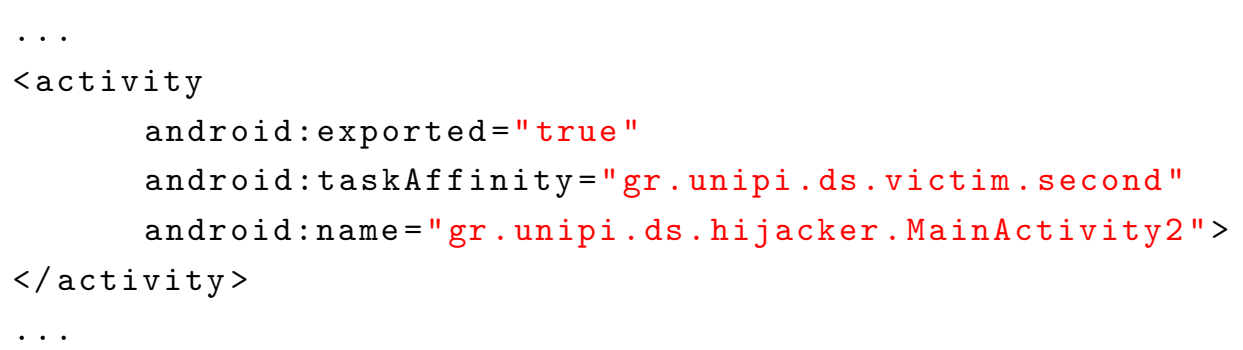

Listing B.3 Example of Manifest File Generated by Anactijax for Malicious Activity Injection Against a Vulnerable Benign Application with Explicitly Defined Tasks.

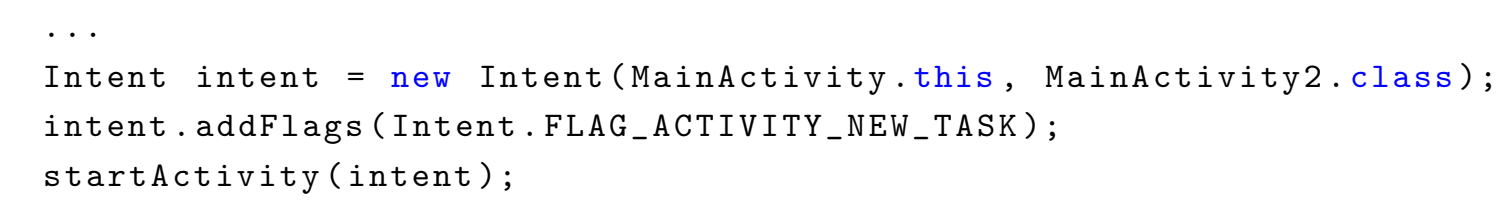

Listing B.4 Example of Code Generated by Anactijax for Malicious Activity Injection Against a Vulnerable Benign Application with Explicitly Defined Tasks.

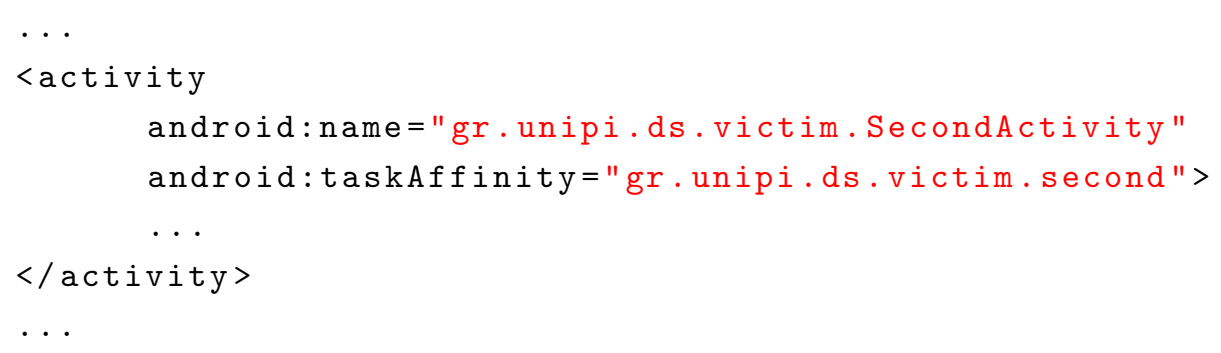

Listing B.5 Example of Manifest File Generated by Anactijax for Vulnerable Benign Application with Explicitly Defined Tasks. 


\section{Index}

Activity Hijacking, 8, 43, 47, 50, 53, 67

Activity Injection, 3, 8, 53

Activity Task Manager Service, 57

Address Space Layout Randomization, 22

Ahead-Of-Time, 14

Android Activity, 15

Android Broadcast Receiver, 15

Android Content Provider, 15

Android Debug Bridge, 31

Android Framework, 14

Android Hidden API, 30

Android Native Development Kit, 14

Android Open Source Project, 29

Android Permissions, 21

Android Runtime, 7

Android Runtime Environment, 13, 14

Android Service, 15

Android System Services, 14

AndroidPacKage, 14

Application Programming Interface, 7, 14, 25

Application Signing, 22

Binder, 13, 16, 57

Central Processing Unit, 13

Certificate Authority, 22

Confused Deputy Attack, 26

Dalvik Executable, 13

Dalvik Virtual Machine, 7, 14
Discretionary Access Control, 20

Explicit Intents, 16, 80

File Based Encryption, 21

Full Disk Encryption, 21

Gatekeeper, 21

Hardware Abstraction Layer, 13

Hardware Security Module, 9, 13, 21

Implicit Intents, 16, 79

Intent, 16, 57

Intent Hijacking, 4, 8, 57, 67

Intent Redirection, 4, 8, 57, 67

Intent-Filter, 58

Inter-Application Communication, 61

Inter-Component Communication, 4, 7, 16, 57,62

Inter-Process Communication, 4, 7, 16, 57, 67

Java Native Interface, 14

Java Reflection, 26, 29

Java Runtime Security Exception, 32

Java Virtual Machine, 7

Just-in-Time, 13

Keymaster, 20

Keystore, 20, 63

Malicious Activity Launch, 57, 67 
Mandatory Access Control, 20

Manifest File, 1, 2, 15, 21, 22, 26, 31, 32, 47,

81,82

No eXecute, 22

Over-privileged Android Applications, 28

Permission Re-Delegation, 26

Public Key Infrastructure, 22

Remote Procedure Calls, 16

SELinux, 20, 63, 64

Software Development Kit, 14, 25

Strongbox, 20

System on Chip, 13

Task Hijacking, 3, 8, 43, 50, 53, 67

Trusted Execution Environment, 9, 13, 20

Trusty OS, 13

TrustZone, 13

Uniform Resource Identifier, 20

User Identifier, 20, 63

Verified Boot, 21

Weaver, 21

Zygote, 14 


\section{Curriculum Vitae}

Christos Lyvas received his B.Sc. from the Department of Informatics, Athens University of Economics and Business (AUEB), in 2013, and his M.Sc. in Digital Systems Security from the Department of Digital Systems, University of Piraeus, in 2015. In 2015 he served the Hellenic army as a security analyst at the Hellenic Army Information Technology Support Center, Hellenic Army General Staff. Since March 2016, he has been a member of the Systems Security Laboratory (SSL) of the University of Piraeus. He has participated as security analyst in several European research programs, such as "SAFERtec", "BIONIC", "CitySCAPE", and "RE-SAMPLE" within the implementation and innovation program "Horizon 2020" of European Commission. His research focuses on mobile applications and operating system's security, malware analysis, and Internet-of-Things security. He is a member of the IEEE and certified ISO 27001:2013 Lead Auditor by TÜV Hellas (TÜV Nord). 



\section{Publications}

\section{Journals}

Christos Kalloniatis, Vasiliki Diamantopoulou, Konstantinos Kotis, Christos Lyvas, Konstantinos Maliatsos, Matthieu Gay, Athanasios G Kanatas, and Costas Lambrinoudakis. Towards the design of an assurance framework for increasing security and privacy in connected vehicles. International Journal of Internet of Things and Cyber-Assurance, 1(3-4):244-266, 2020. ISSN 2059-7967. doi: https://doi.org/10.1504/IJTCA.2020.112574. URL https://www . inderscienceonline.com/doi/ abs/10.1504/IJITCA.2020.112574.

Christos Lyvas, Costas Lambrinoudakis, and Dimitris Geneiatakis. Dypermin: Dynamic permission mining framework for android platform. Computers \& Security, 77:472-487, 2018. ISSN 0167-4048. doi: https://doi.org/10.1016/j.cose.2018.05.007. URL https://www. sciencedirect. com/science/ article/pii/S0167404818304954.

Christos Lyvas, Costas Lambrinoudakis, and Dimitris Geneiatakis. On android's activity hijacking prevention. Computers \& Security, 111:102468, 2021. ISSN 0167-4048. doi: https: //doi.org/10.1016/j.cose.2021.102468. URL https://www . sciencedirect. com/science/article/ $\mathrm{pii} / \mathrm{S} 0167404821002923$.

Christos Lyvas, Christoforos Ntantogian, and Christos Xenakis. [m]allotropism: a metamorphic engine for malicious software variation development. International Journal of Information Security, pages 1-18, 2021. doi: https://doi.org/10.1007/s10207-021-00541-y. URL https ://link . springer . com/article/10.1007/s10207-021-00541-y.

Christos Lyvas, Costas Lambrinoudakis, and Dimitris Geneiatakis. IntentAuth: Securing Android's Intent Based Inter-Process Communication. International Journal of Information Security, 2021 (Under Minor Revision). 


\section{Conferences}

Vasiliki Diamantopoulou, Christos Kalloniatis, Christos Lyvas, Konstantinos Maliatsos, Matthieu Gay, Athanasios Kanatas, and Costas Lambrinoudakis. Aligning the concepts of risk, security and privacy towards the design of secure intelligent transport systems. In Computer Security, pages 170-184. Springer, 2020. doi: https://doi.org/10.1007/978-3-030-64330-0_11. URL https: //link.springer.com/chapter/10.1007/978-3-030-64330-0_11.

Christos Lyvas, Nikolaos Pitropakis, and Costas Lambrinoudakis. The far side of mobile application integrated development environments. In International Conference on Trust and Privacy in Digital Business, pages 111-122. Springer, 2016. doi: https://doi.org/10.1007/978-3-319-44341-6_8. URL https://link. springer.com/chapter/10.1007/978-3-319-44341-6_8.

Konstantinos Maliatsos, Christos Lyvas, Panagiotis Pantazopoulos, Costas Lambrinoudakis, Athanasios Kanatas, Matthieu Gay, and Angelos Amditis. Standardizing security evaluation criteria for connected vehicles: A modular protection profile. In 2019 IEEE Conference on Standards for Communications and Networking (CSCN), pages 1-7. IEEE, 2019. doi: https://doi.org/10.1109/ CSCN.2019.8931344. URL https://ieeexplore. ieee.org/document/8931344.

Nikolaos Pitropakis, Christos Lyvas, and Costas Lambrinoudakis. The greater the power, the more dangerous the abuse: facing malicious insiders in the cloud. In International Conference on Cloud Computing, GRIDs, and Virtualization, Track Security and Privacy in Cloud Computing (SEPRICC 2017), pages 156-161. IARIA Xpert Publishing Services, 2017. 